\title{
An Overview of Instability and Fingering During Immiscible \\ Fluid Flow in Porous and \\ Fractured Media
}

Manuscript Completed: November 1994

Date Published: April 1995

Prepared by

G. Chen, M. Taniguchi, ${ }^{*}$ S. P. Neuman

T. J. Nicholson, NRC Project Manager

Department of Hydrology and Water Resources

The University of Arizona

Tucson, AZ 85721

\section{Prepared for}

Division of Regulatory Applications

Office of Nuclear Regulatory Research

U.S. Nuclear Regulatory Commission

Washington, DC 20555-0001

NRC Job Code L1283

*Department of Earth Sciences, Nara University of Education, Nara 630, Japan 
For sale by the U.S. Government Printing Office

Superintendent of Documents, Mail Stop: SSOP, Washington, DC 20402-9328

ISBN $0-16-048053-1$ 


\section{DISCLAIMER}

This report was prepared as an account of work sponsored by an agency of the United States Government. Neither the United States Government nor any agency thereof, nor any of their employees, make any warranty, express or implied, or assumes any legal liability or responsibility for the accuracy, completeness, or usefulness of any information, apparatus, product, or process disclosed, or represents that its use would not infringe privately owned rights. Reference herein to any specific commercial product, process, or service by trade name, trademark, manufacturer, or otherwise does not necessarily constitute or imply its endorsement, recommendation, or favoring by the United States Government or any agency thereof. The views and opinions of authors expressed herein do not necessarily state or reflect those of the United States Government or any agency thereof. 


\section{DISCLAIMER}

Portions of this document may be illegible in electronic image products. Images are produced from the best available original document. 


\begin{abstract}
Wetting front instability is an important phenomenon affecting fluid flow and contaminant transport in unsaturated soils and rocks. It causes the development of fingers which travel faster than would a uniform front and thus bypass much of the medium. Water saturation and solute concentration in such fingers tend to be higher than in the surrounding medium. During infiltration, fingering may cause unexpectedly rapid arrival of water and solute at the water-table. This notwithstanding, most models of subsurface flow and transport ignore instability and fingering. In this report, we survey the literature to assess the extent to which this may or may not be justified. Our overview covers experiments, theoretical studies, and computer simulations of instability and fingering during immiscible two-phase flow and transport, with emphasis on infiltration into soils and fractured rocks. Our description of instability in an ideal fracture (Hele-Shaw cell) includes an extension of existing theory to fractures and interfaces having arbitrary orientations in space. Our discussion of instability in porous media includes a slight but important correction of existing theory for the case of an inclined interface. We conclude by outlining some potential directions for future research. Among these, we single out the effect of soil and rock heterogeneities on instability and preferential flow as meriting special attention in the context of nuclear waste storage in unsaturated media.
\end{abstract}




\section{TABLE OF CONTENTS}

Page

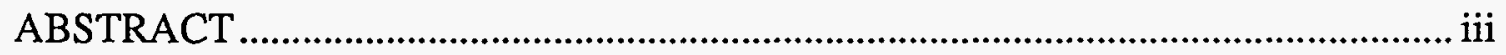

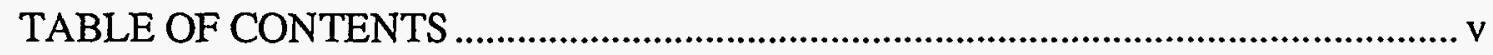

LIST OF FIGURES............................................................................................. vii

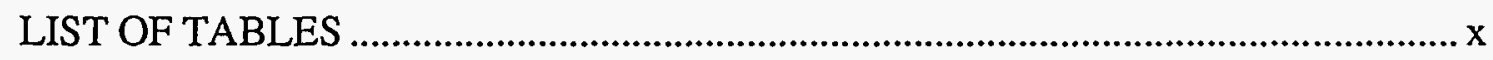

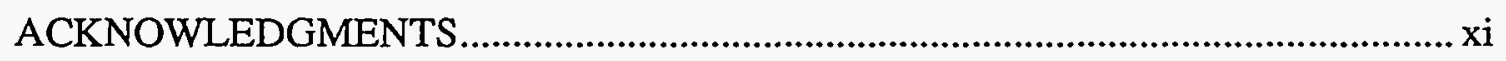

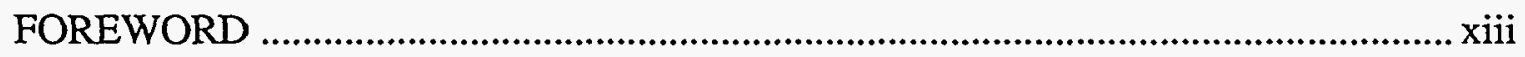

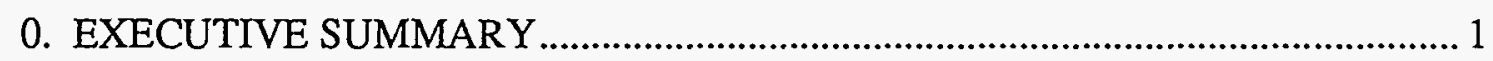

1. INTRODUCTION

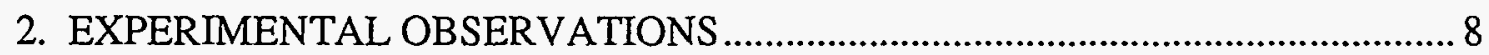

2.1 Immiscible Displacement in Hele-Shaw Cells .............................................. 8

2.2 Immiscible Displacement in Porous Media ……………........................... 20

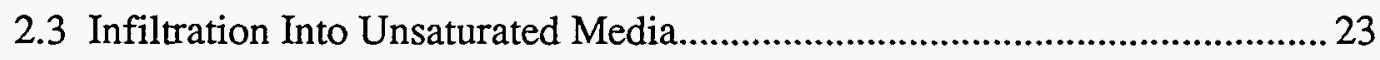

2.3.1 Laboratory Infiltration Into Layered Media..................................... 26

2.3.2 Laboratory Infiltration Into Nonlayered Media ................................. 34

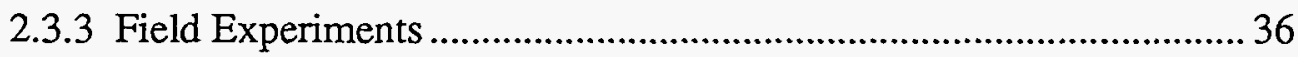

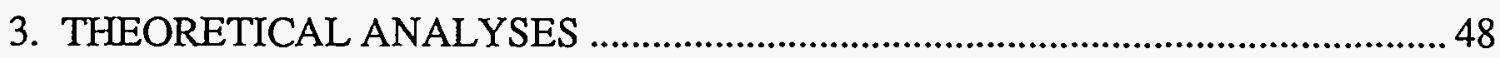

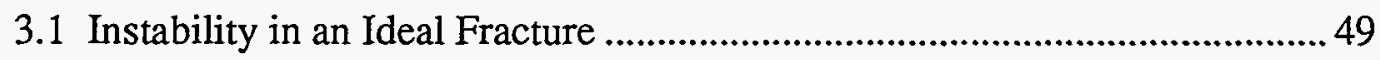

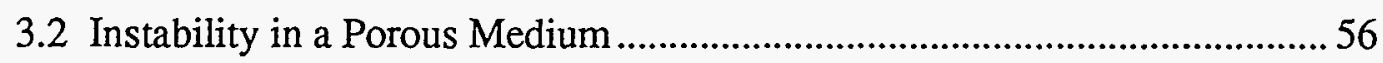

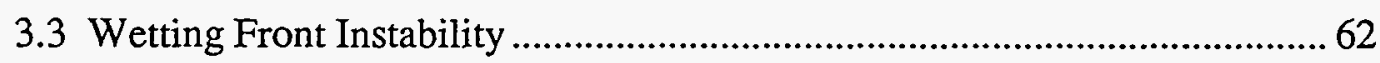

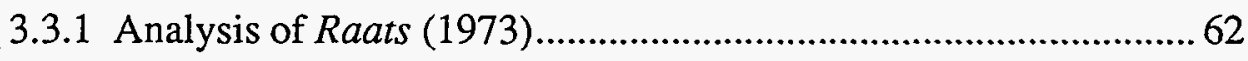

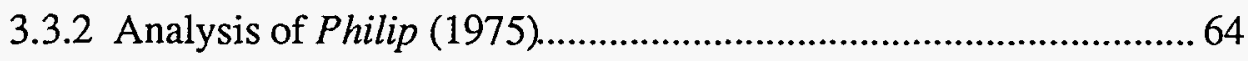

3.3.3 Analyses of Parlange and Hill (1976) and

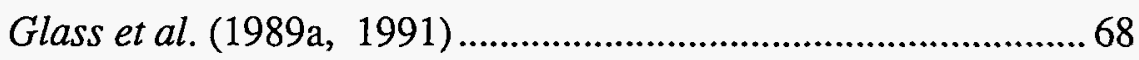

3.3.4 Analysis of Diment et al. (1982)................................................... 72

3.3.5 Analysis of Hillel and Baker (1988)........................................... 73

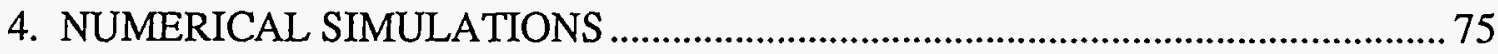

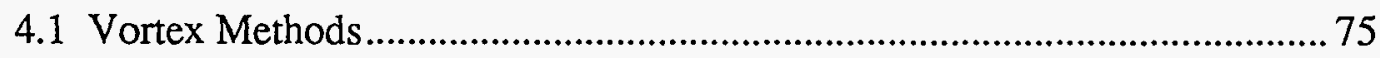

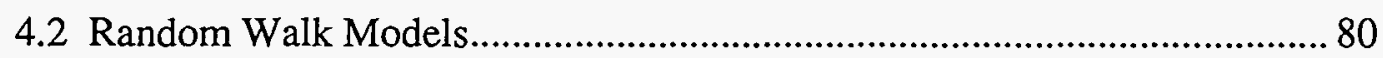




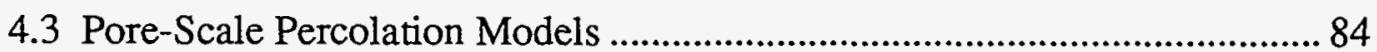

4.4 Conventional Numerical Methods ............................................................. 90

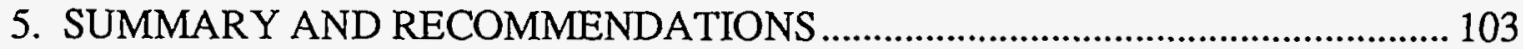

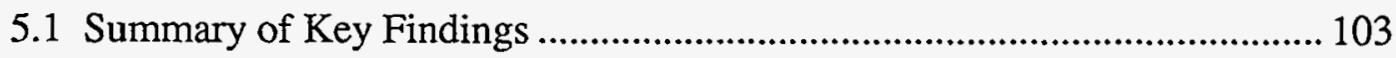

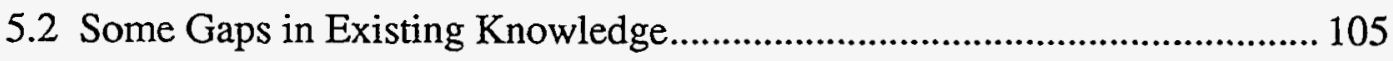

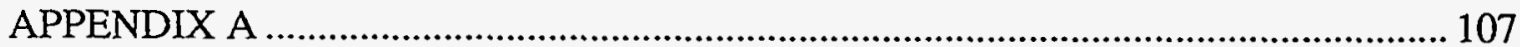

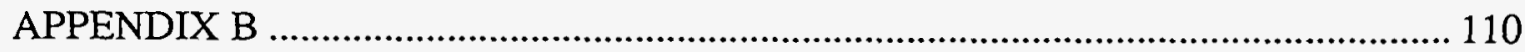

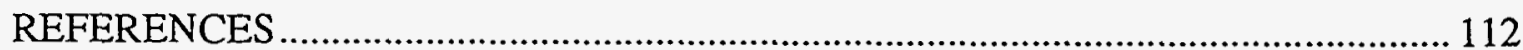




\section{LIST OF FIGURES}

Figure

Page

2.1 Sketch of Hele-Shaw cell used by Saffman and Taylor (1958)........................ 9

2.2 Stable and unstable situations in vertical displacement.................................... 11

2.3 Development of instability and fingering as air displaces glycerine downward in a Hele-Shaw cell

2.4 Measured values of $r$ for water penetrating into two oils ................................ 13

2.5 Instability of the interface between a water-glycerine mixture and oil in an inclined Hele-Shaw cell............................................................................... 15

2.6 Fingering pattern in a Hele-Shaw cell at different stages of displacement

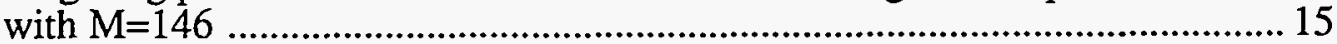

2.7 Growing and neutral modes of an instability .................................................. 17

$2.8 \quad$ Sequence of growth stages for fingering with tip splitting .............................. 17

2.9 Fingering in a vertical Hele-Shaw cell with $\mathrm{Ca}^{\prime}=5 \times 10^{4}$ when air displaces silicone oil upward

2.10 Fingering in a horizontal Hele-Shaw cell with $C a^{\prime}=5 \times 10^{4}$ when air displaces silicone oil......................................................................................... 21

$2.11 \quad$ Fingering in porous media ......................................................................... 24

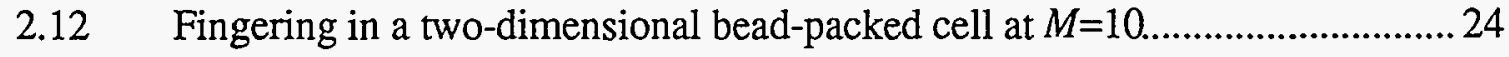

2.13 Development of fingers in a coarse sand due to infiltration of water through an overlying fine sand................................................................................ 28

$2.14 \quad$ Sketch of observed finger translation .................................................................. 30

2.15 Effect of infiltration rate on finger width and propagation rate .......................30

2.16 Cross-sections showing fingers in three dimensional experiments.

2.17 Finger movement through a dry sand into a wetted sand .................................33

2.18 Preliminary experiment demonstrating the effects of slight heterogeneities on finger meandering and merger in a two-layer soil.............. 33

2.19 Development of dyed infiltration front in a heterogeneous medium ................ 35

2.20 Fingering during redistribution in dry sand following infiltration.....................37 
2.21 Wetting front instability during redistribution in a natural fracture

2.22 Finger development during redistribution in an initially dry artificial fracture at $\delta=139^{\circ}, L_{a}=9.43 \mathrm{~cm}$

2.23 Effect of ponding depth $\left(L_{a}\right)$ on finger development in an artificial fracture during redistribution at $\delta=139^{\circ}$

2.24 Effect of $\delta$ on finger development during redistribution in an artificial

fracture

2.25 Isolated zones of dyed water at $100 \mathrm{~cm}$ depth after infiltration into a soil column at Windsor, Connecticut

2.26 Horizontal dye patterns at depths of (a) $50 \mathrm{~cm}$, (b)

$70 \mathrm{~cm}$, (c) $90 \mathrm{~cm}$, (d) $115 \mathrm{~cm}$, and (e) $140 \mathrm{~cm}$.

2.27 Percent blue area versus soil depth in five plots ................................... 44

Sketch of disturbed interface.................................................................. 48

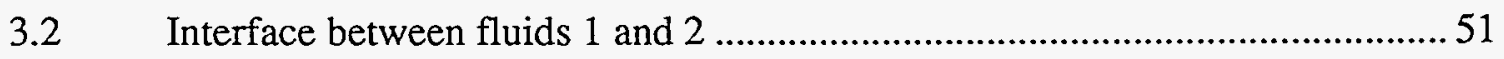

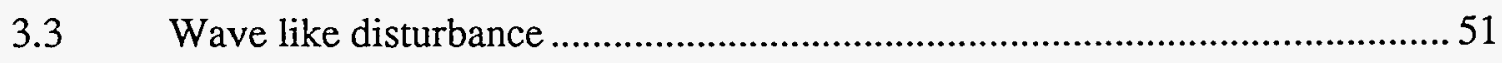

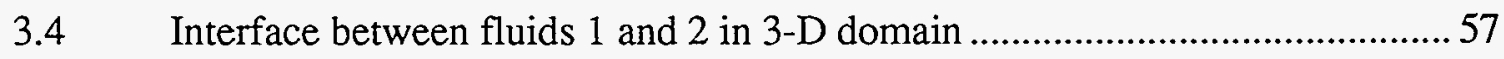

4.1 Sketch defining notation for vortex equations ...................................... 76

4.2 Stages of interface evolution using vortex-in-cell method............................ 79

4.3 VIC prediction of finger development from (a) a single sine wave and

(b) two superimposed sine waves.

4.4 Point vortex results with $A=1$ and (a) $C a^{\prime}=530$; (b) $C a^{\prime}=833$;

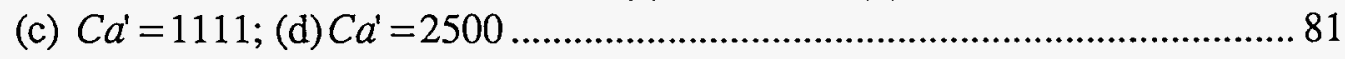

Fractal structures produced by DLA ................................................... 82

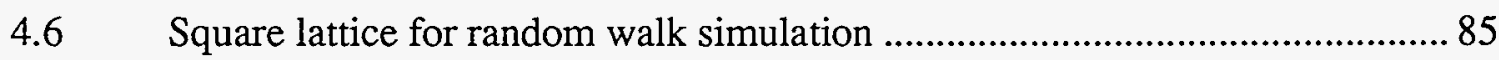

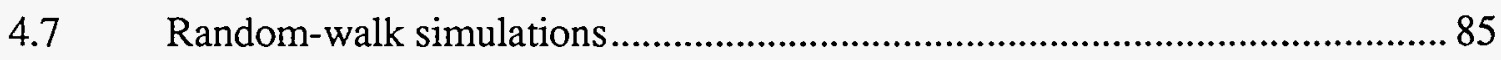

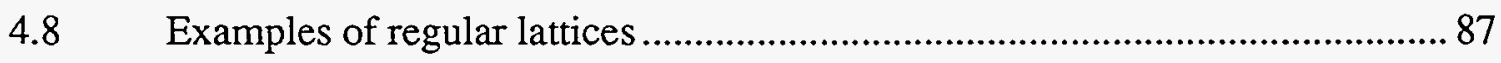

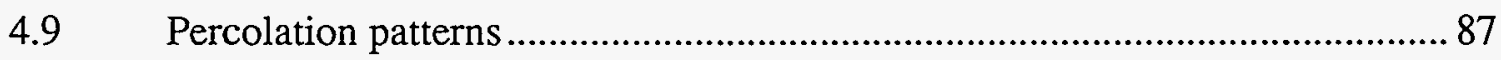

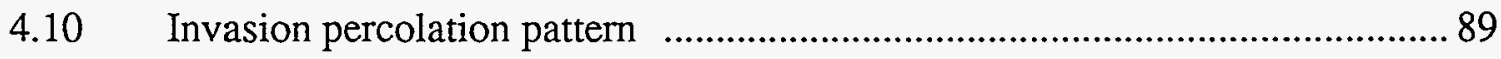

4.11 Invasion percolation of water into an air-dry rough-walled fracture ..............91

4.12 Contours of water saturation during displacement of oil at four equally spaced time steps. 
4.13 Interpretations of (a) change in saturation as a probability density function and (b) random selection of gridblock

4.14 Water saturations at breakthrough during displacement of oil by combined finite difference and Monte Carlo simulations.

4.15 Flux-corrected simulation of oil displacement by water at $M=10$.............97

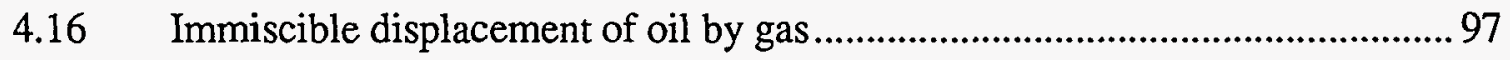

4.17 Effect of $D P$ and spatial correlation of $\log$ permeability on water saturation profiles

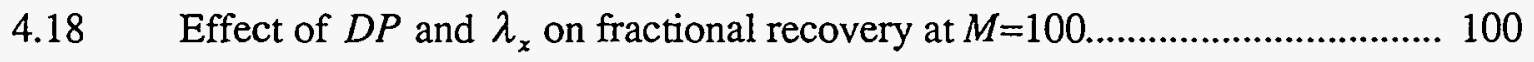

4.19 Change in volumetric water content after 50 days of infiltration into a nonuniform soil, and tracer concentrations with and without dispersion 102 


\section{LIST OF TABLES}

\section{Page}

\section{Table}

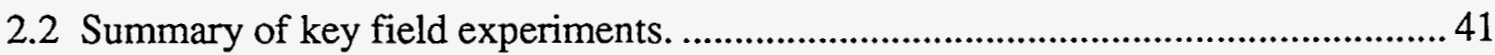




\section{ACKNOWLEDGMENTS}

The present overview has been performed by The University of Arizona, Department of Hydrology and Water Resources for the U. S. Nuclear Regulatory Commission (NRC) under contract Job Code L1283. Project monitor for the NRC is Mr. Thomas J. Nicholson, and Principal Investigator is Dr. Shlomo P. Neuman. Support for this work by the U. S. Nuclear Regulatory Commission is gratefully acknowledged.

We thank the following agencies and individuals for granting us written permission to reproduce their copyrighted materials: American Geophysical Union, American Institute of Physics, American Physical Society, American Society of Agricultural Engineers, American Society of Civil Engineers, Cambridge University Press, Elservier Science Publishers, Kluwer Academic Publishers, Lewis Publishers, Plenum Press, Society of Petroleum Engineers, Soil Science Society of America, Williams \& Wilkins, and H. Aref, L. W. Dekker, R. J. Glass, G. M. Homsy, J. Koplik, R. Lenormand, E. J. Peters, P. G. Saffman, and T. Maxworthy. 



\section{FOREWORD}

This technical report was prepared by the University of Arizona under their research project with the Waste Management Branch in the Office of Nuclear Regulatory Research (Job Code L1283). This report presents an overview of instability and fingering phenomena during the displacement of one immiscible fluid by another. Its focus is on the displacement of air by water during the downward propagation of wetting fronts through heterogeneous unsaturated soils and fractured rocks due to infiltration at the ground surface. This report summarizes and expands upon findings in the published literature which are relevant to the characterization of sites to be considered for disposal of nuclear waste in unsaturated geologic media, such as the Yucca Mountain site. It identifies gaps in knowledge which are of similar relevance and points to areas that may warrant further research.

This report is not a substitute for NRC regulations, and compliance is not required. The approaches and/or methods described in the NUREG/CR-6308 are provided for information only. Publication of this report does not necessarily constitute NRC approval or agreement with the information contained herein. 


\section{EXECUTIVE SUMMARY}

This report presents an overview of published experimental, theoretical and numerical work on instability and fingering during immiscible fluid displacement in porous and fractured media, particularly wetting front instability in unsaturated soils and rocks. Instability and fingering during miscible displacement is outside the scope of our report. The published literature suggests that wetting front instability is a commonly occurring phenomenon which has an important effect on fluid flow and contaminant transport in unsaturated soils and rocks. As such, it is of potential interest in the context of nuclear waste disposal in unsaturated geologic media. It causes the development of fingers which travel faster than would be anticipated on the basis of Darcy's law. This may cause water and contaminants to reach the water table faster than would otherwise be the case. The phenomenon occurs in both uniform and nonuniform media in response to either ponding or sprinkling at the surface. It also takes place during moisture redistribution in the wake of an infiltration event.

Wetting front instability is a special case of interface instability during immiscible fluid displacement in porous and fractured media. The phenomenon is triggered by unfavorable differences between the viscosities and densities of the two fluids across their interface. Viscous forces have a stabilizing influence when a more viscous fluid displaces a less viscous fluid, and a destabilizing influence when a less viscous fluid displaces a more viscous fluid. Both effects increase with the mean propagation speed of the interface. Gravity has a stabilizing influence when the denser fluid is at the bottom, and a destabilizing influence otherwise, regardless of mean flow direction or speed.

When both viscous and gravitational forces act to stabilize the interface, the latter is unconditionally stable. When both of these forces act to destabilize the interface, it is unconditionally unstable. When viscosity is stabilizing and gravity is destabilizing, the interface is stable provided that its mean speed exceeds some critical value. When viscosity is destabilizing and gravity is stabilizing, the interface is stable on condition that its mean speed is below a critical value.

During the downward propagation of a wetting front in an unsaturated soil or rock environment, viscosity acts as a stabilizing force and gravity as a destabilizing force. Hence for instability to occur, the mean speed of front propagation must be less than some critical value. For a sharp wetting front with a uniform moisture profile above and below, 
this critical speed is equal to the ratio between hydraulic conductivity above the front and the drop in water content across it.

Surface tension has a stabilizing effect on the interface between two immiscible fluids in all cases. Suppose that the interface is perturbed slightly into a composite wave form containing diverse wave lengths (and spatial frequencies) of very small amplitude. If the interface is unstable and there is no surface tension, the amplitudes of all these perturbations (incipient fingers) can initially grow at rates that increase as their wavelengths decrease (frequencies increase). If surface tension is active, only perturbations with wavelengths above some critical value (frequencies below some critical value) can grow, all others decay. The critical wavelength increases with surface tension (for a sharp wetting front with a uniform moisture profile above and below, it decreases as the drop in water content across the front increases). The fastest growing perturbation has a wavelength that exceeds critical by a constant factor. The corresponding fingers are thought to appear first in an experiment and to dominate their neighbors. Smaller fingers often coalesce into one or more dominant fingers.

Under uniform mean flow in homogeneous media, established fingers tend to elongate linearly with time. Their rate of elongation increases with mean flow rate and viscosity contrast but decreases with surface tension.

Shielding occurs when larger fingers outgrow their smaller neighbors and spread laterally to inhibit their growth. The widening of dominant fingers by shielding reduces surface tension which may render them unstable. This, in turn, may cause the fingers to split (bifurcate) at their tips into narrower branches which are stable due to increased surface tension. Shielding then favors one of these branches which widens, becomes unstable, and so on. The cycle of shielding, spreading and splitting may repeat itself periodically. It is not commonly observed when wetting fronts propagate downward through unsaturated media.

When capillary action is weak and/or mean flow rate is high, bifurcation may continue to yield a contorted, dendritic interface with a randomly fractal geometry.

Theoretical considerations published earlier by one of us suggest that, in a HeleShaw cell (or ideal fracture with smooth impermeable walls separated by a constant aperture) upon the onset of instability, the interface is generally not perpendicular (and fingers are generally not parallel) to the direction of mean flow unless the latter coincides with the dip of the cell (or fracture). We have shown theoretically in this report that, in a 
three-dimensional porous medium upon the onset of instability, the interface is generally not perpendicular (and fingers are generally not parallel) to the mean direction of flow unless the latter is vertical. This is contrary to Chuoke et al. (1959) who allowed mean flow to be other than vertical but conjectured that the interface would remain at right angle to this flow vector.

Spatial variations in medium permeability are conducive to the preferential development of fingers along paths of least resistance to flow. Instability in such heterogeneous media may occur under conditions that would not be favorable for the development of fingers in equivalent uniform media. This is true on the pore scale, on the laboratory scale, and on the field scale where preferential flow of water and solutes is commonly observed. A wetting front becomes unstable as it descends from a fine-grained into a coarse-grained soil. The same happens as it reaches a critical depth when permeability increases systematically downward. In soils that are wetted nonuniformly (say by antecedent fingers), new fingers tend to move preferentially through the wetted regions because these pose the least resistance to flow.

A sharp wetting front is more prone to become unstable than a diffuse front; conditions which cause instability in a poorly-graded (usually coarse) soil may therefore give rise to a stable front in a well graded (usually fine) soil. Other factors which contribute to the development of unstable wetting fronts include water repellency of the soil and compression of air ahead of the front.

Issues concerning the onset of instability are often amenable to first-order mathematical analysis by linearization. As fingers grow, their behavior becomes so strongly nonlinear that numerical analysis is usually required. Numerical methods that have proven capable of simulating the development of fingers under select conditions include point vortex and vortex-in-cell techniques, random walk models, pore-scale percolation models, Eulerian-Lagrangian methods, and the flux corrected scheme. Among these, the latter two approaches seem better suited for the simulation of unstable fluid interfaces in heterogeneous media than the rest.

Much is yet to be learned about various aspects of instability and finger development during immiscible displacement. We believe that the effect of soil and rock heterogeneities on the instability of wetting fronts merits special attention in the context of nuclear waste storage in unsaturated geologic environments. In particular, little is presently known about the extent to which porous rocks containing fractures, faults and 
bedding planes (as is the case at Yucca Mountain in Nevada) may facilitate or retard the development of unstable fingers and their rapid propagation (vertically or otherwise) to the water table. What effects do various parameters which define the spatial variability of rock properties (fracture, fault and bedding plane geometries; mean, variance and correlation scales of permeabilities in diverse lithologic and structural units) have on the onset of instabilities under various antecedent moisture conditions and surface infiltration scenarios on relevant field scales? In what directions, and at what rates, might fingers propagate after instability has set in? How long, wide and numerous will such fingers be? What will be their locations and spacings? Will they occur exclusively in fractures or will they invade matrix blocks? How is this related to the mass rate of water and solute propagation through the geologic medium? To address the issue of solute transport one may have to consider not only immiscible but also miscible displacement processes such as dispersion, matrix diffusion and sorption. What effect do these have on the stability of solute fronts under unsaturated conditions? How might elevated temperatures in parts of the flow field affect such behaviors? Might condensed water vapors form potentially unstable moisture fronts? What observations and experiments might feasibly help reveal these and related phenomena in the field? Our overview suggests that these questions address realistic problems which have a direct bearing on our understanding of unsaturated fluid flow and contaminant transport in complex geologic media. 


\section{INTRODUCTION}

Instability and fingering are of potential interest in the context of nuclear waste disposal in geologic media. Instability occurs when small perturbations in the flow pattern grow without limit with time. In particular, small perturbations along interfaces between immiscible fluids, such as wetting fronts that form during infiltration into unsaturated soils and rocks, may give rise to fingers which move faster than would be anticipated on the basis of Darcy's law. This may cause water and contaminants to reach the water table faster than would otherwise be the case.

Instability and fingering have been of concern to the petroleum industry. Following the primary recovery of oil, where ambient formation pressures provide the primary energy to drive oil toward production wells, a large portion of the petroleum reserves may remain in the reservoir, untapped. To extract this residual oil, water or other fluids are sometimes injected to help drive the oil toward production wells. Experience has shown that such secondary recovery, or immiscible flooding, may bypass much of the residual oil due to the formation of fingers which result largely from a viscosity difference between the injected fluid and oil. These viscous fingers have an adverse effect on the efficiency of secondary recovery processes. Tertiary recovery involves the injection of $\mathrm{CO}_{2}$ gas or other surfactants which reduce surface tension and thus help free residual oil from small pores in the rock. Here the injected fluids mix with the resident oil and the displacement process is thus miscible. If fingering occurs during miscible displacement, the efficiency of tertiary recovery is reduced. Instability associated with miscible processes is beyond the scope of this report.

Wetting front instability and fingering may be important for the prediction of subsurface contaminant transport. It has been common to assume that spatial and temporal variations in near-surface moisture conditions die out rapidly with depth. This implies that wetting fronts and contaminants propagate downward at a relatively slow rate, controlled by Darcy's law. It further implies that contaminants come into contact with immobile waters and/or soils everywhere along the path of a uniformly advancing wetting front, allowing for maximum retention due to diffusion and sorption within the immobile soil-water mixture. There is a growing body of evidence to suggest that this is not always the case. Of particular relevance here are observations such as those recently reported at Yucca Mountain, Nevada (Quade and Cerling, 1990), where chlorine-36 and polymerbased drilling waters have provided evidence for unusually rapid transport in the vadose 
zone. Is such rapid, preferential flow due to instability, fracture and rock matrix heterogeneity, or both? This is one major question which motivates our overview, and our research, on the subject.

Early work on the instability of immiscible fluid interfaces was reported by Hill (1952). The first rigorous hydrodynamic stability analysis, considering surface tension, was performed by Saffman and Taylor (1958) on a horizontal interface in a vertical HeleShaw cell. A similar analysis was done independently on porous media by Chuoke et al. (1959). These analyses showed that viscosity and density differences can have either a stabilizing or a destabilizing effect, depending on which fluid displaces which in what direction. Surface tension has a stabilizing influence in all cases. The relative importance of these three factors is reflected in the magnitude of three dimensionless groups: the capillary number $C a=\mu_{1} U / T$ which measures the importance of viscosity relative to surface tension, the viscosity ratio $M=\mu_{1} / \mu_{2}$ between the displaced and displacing fluids, and the Darcy-Rayleigh number $D R=\left(\rho_{1}-\rho_{2}\right) g k /\left[\left(\mu_{1}+\mu_{2}\right) U\right]$ which measures the importance of buoyancy relative to viscosity. Here $\mu, \rho, g, k, U$, and $T$ represent dynamic viscosity, density, acceleration due to gravity, permeability, displacement speed and surface tension, respectively, while the subscripts 1,2 designate respectively the displaced and displacing fluids. These definitions are in part intuitive; some recent authors prefer to use the modified capillary number $C a^{\prime}=\mu_{1} U /(k T)$ and mobility ratio $A=\left(\mu_{1}-\mu_{2}\right) /\left(\mu_{1}+\right.$ $\mu_{2}$ ) which arise from a mathematical statement of the problem. The literature describes extensive experimental and numerical studies which investigate the effect of these dimensionless groups on instability and the evolution of fingers under a variety of conditions. Some of this work has been summarized by Homsy (1987).

Unstable wetting fronts during infiltration into soils have been first observed in the field by Deecke (1906) and later in the laboratory by Tabuchi (1961) and Miller and Gardner (1962). The phenomenon has been investigated systematically by Hill and Parlange (1972) who had conducted experiments on fingering at the interface between two soil layers with different textures. Their work has been instrumental in alerting soil physicists to the possibility that preferential flow may result from wetting front instability. Early theoretical explanations for the phenomenon have been proposed by Raats (1973) and Philip (1975). Among other findings, these authors have pointed out that wetting front instability arises not only due to layering but also due to a gradual increase in permeability with depth, enhanced air pressure ahead of the wetting front, redistribution of water, and other causes. 
In this report we offer a thorough overview of the literature on instability and fingering during immiscible displacement. Though our overview is broad, its main focus is on the infiltration of water into unsaturated soils and fractured rocks. Our overview complements, but does not supersede, earlier reviews by Wooding and Morel-Seytoux (1976) and Kueper and Frind (1988) on multiphase fluid flow in porous media, Bensimon et al. (1986) and Saffman (1986, 1991) on viscous fingering in Hele-Shaw cells, Homsy (1987) on viscous fingering in uniform porous media and Hele-Shaw cells, and brief reviews of wetting front instability by Hillel $(1987,1993)$. Our report differs from these reviews both in its focus and in its attempt to be self-explanatory. This means that we present the subject in a way which might serve as a tutorial for persons who are not intimately familiar with the subject. Our purpose is to (a) establish the extent to which the subject should form the focus of future regulatory research, (b) provide the necessary technical background for such research, and (c) point toward directions that such research might fruitfully take in the future.

The report starts with a survey of experimental observations which will supply the reader with an intuitive understanding of instability, fingering, and some of their controlling factors. It then proceeds to outline available theories of instability, followed by a description of relevant numerical experiments. The report ends with a summary of findings to date and suggestions for future research. 


\section{EXPERIMENTAL OBSERVATIONS}

In this chapter we review some of the most important experiments to date concerning instability and fingering between two immiscible fluids with different viscosities and densities. Section 2.1 describes experiments in Hele-Shaw cells, and Section 2.2 in porous media. Wetting front instability and fingering during water infiltration into unsaturated media is discussed separately in Section 2.3.

\subsection{Immiscible Displacement in Hele-Shaw Cells}

Hele-Shaw cells were first described by Hele-Shaw in 1898. They are of interest because they constitute convenient experimental analogous of two-dimensional flow in porous media and rock fractures. A Hele-Shaw cell typically consists of two rectangular plates separated by a small gap, or aperture. The plates may be vertical, horizontal, or inclined and flow in the cell may be directed up, down, or sideways. A partial diagram of the Hele-Shaw cell used in the classical experiments of Saffman and Taylor (1958) is shown in Figure 2.1.

The analogy between flow in a Hele-Shaw cell and flow in a porous medium is well established (e.g., Lamb, 1932). By integrating the steady state Stokes equation for slow (small Reynolds number) viscous flow of an incompressible homogeneous Newtonian fluid under isothermal conditions between the two plates, subject to no-slip conditions at solid-fluid interfaces, one obtains

$$
\nabla h=-\frac{12}{b^{2}} \frac{\mu}{\rho g} \mathbf{u}
$$

where $h$ is total fluid potential or head, $\mathbf{u}$ is average velocity vector, $\mu$ is dynamic viscosity and $b$ is aperture. This is equivalent to Darcy's law with an intrinsic permeability equal to $b^{2} / 12$. Mass balance requires that

$$
\nabla \cdot \mathbf{u}=0 \text { or } \quad \nabla^{2} h=0
$$

as in porous media. However, the analogy is not perfect in that the Hele-Shaw cell does not mimic the complex topology and geometry of openings in real porous and fractured media. Yet such details may be very important when it comes to spatial variability and anisotropy of permeability, two-phase flow, instability and fingering, or solute transport. Nevertheless, an understanding of instability and fingering in Hele-Shaw cells is very useful for the understanding of these phenomena in natural soils and rocks. 

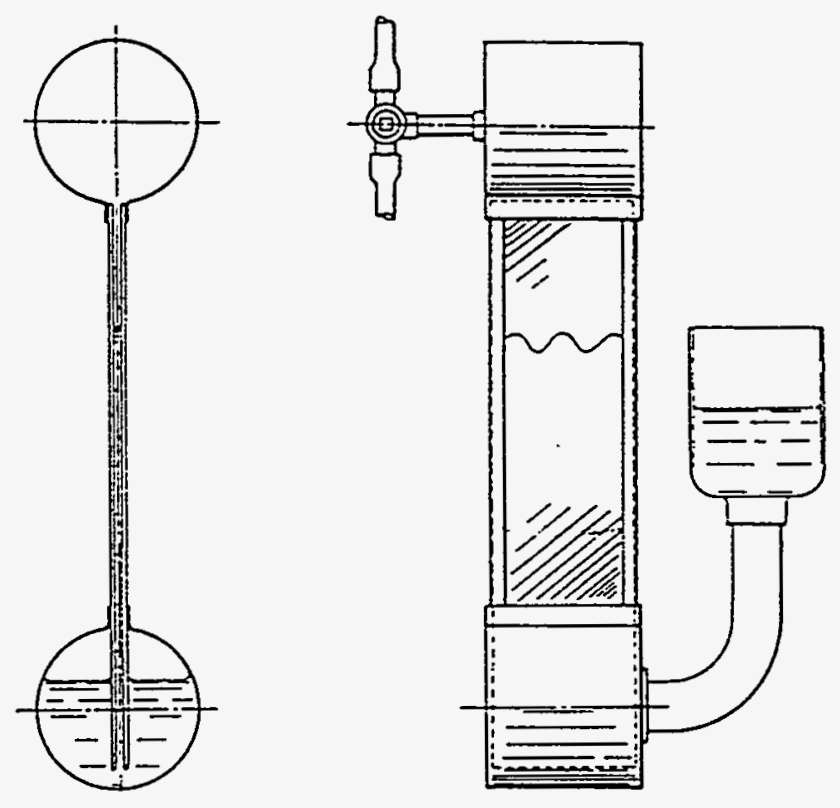

Figure 2.1. Sketch of Hele-Shaw cell used by Saffman and Taylor (1958). 
In the classical experiments of Saffman and Taylor (1958) the Hele-Shaw cell was either vertical or horizontal. The narrow cell was connected at each end to a vessel containing one of the two fluids (Figure 2.1). A potential gradient along the cell was produced by applying either pressure or suction to the air above the fluid in one of the end vessels while maintaining atmospheric air pressure in the other vessel. Three experiments were performed, the first involving air and glycerine in a vertical cell. Initially, a sharp horizontal interface separated the two fluids. When air was allowed to displace glycerine downward, the interface became unstable once the average displacement velocity reached a critical value. When glycerine was placed above the air while flow was directed downward, instability developed when the velocity was less than critical. Figure 2.2 presents a summary of these and related experiments by Chuoke et al. (1959), including miscible displacement experiments by Hill (1952). We see that, regardless of flow direction, the interface is unconditionally stable as long as the denser fluid is at the bottom and a more viscous fluid displaces a less viscous fluid. If both of these relationships are reversed, the interface becomes unconditionally unstable. This suggests that viscous resistance has a stabilizing effect when a more viscous fluid displaces a less viscous fluid, and a destabilizing effect when a less viscous fluid displaces a more viscous fluid. Since viscous resistance is proportional to velocity, both effects increase with velocity. On the other hand, gravity has a stabilizing effect when the denser fluid is at the bottom, and a destabilizing effect otherwise, regardless of flow direction or velocity. Therefore, when both viscous and gravitational forces act to stabilize the interface, the latter is unconditionally stable; when both act to destabilize it, the interface becomes unconditionally unstable. When gravity is stabilizing and viscosity is destabilizing, the interface is stable for $U<U_{c}$ where $U_{c}$ is a critical speed of interface motion; when gravity is destabilizing and viscosity is stabilizing, the interface is stable for $U>U_{c}$. If flow is horizontal, gravity plays no role and instability occurs solely when a less viscous fluid displaces a more viscous fluid. As will be seen later, this observed behavior is predicted by theory.

An example of finger development in the Saffman-Taylor (1958) experiments is shown in Figure 2.3. Figure 2.3a depicts the early stage where glycerine has been displaced a few centimeters downward by the overlying air. Incipient instability manifests itself through the formation of low-amplitude ondulations of various wavelengths. Figure $2.3 \mathrm{~b}$ illustrates a later stage of the displacement in a different experiment where some of these waves have grown to form elongated fingers, while others have degenerated. 


\section{Upward Flow}

unconditionally unconditionally stable when stable when stable unstable $U<U_{c} \quad U>U_{c}$
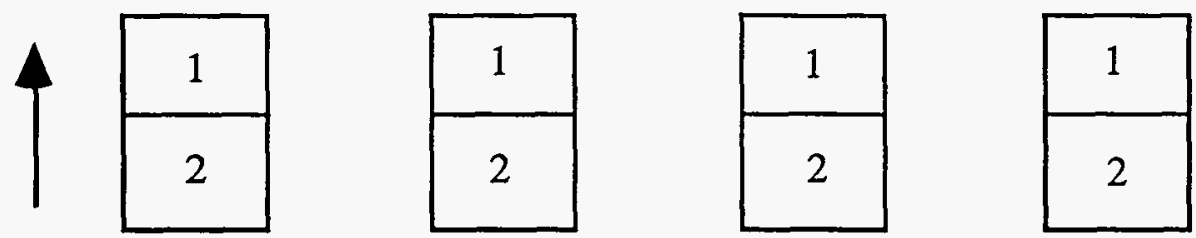

$$
\mu_{2}>\mu_{1}, \rho_{2}>\rho_{1}
$$$$
\mu_{2}<\mu_{1}, \rho_{2}<\rho_{1}
$$$$
\mu_{2}<\mu_{1}, \rho_{2}>\rho_{1}
$$

$$
\mu_{2}>\mu_{1}, \rho_{2}<\rho_{1}
$$

(a)

(b)

(c)

(d)

\section{Downward Flow}

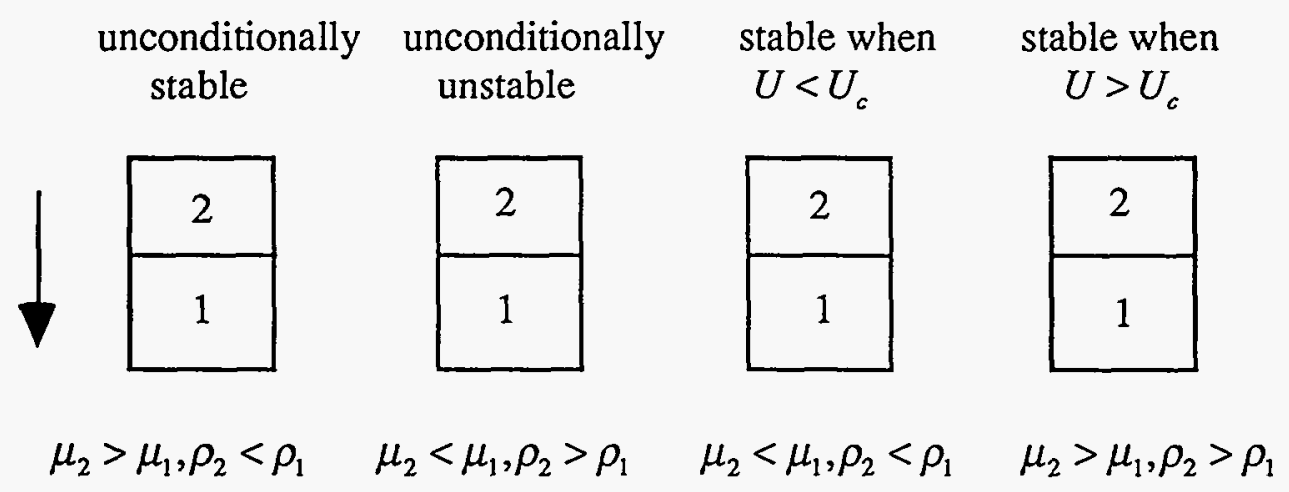

(e)

(f)

(g)

(h)

Figure 2.2. Stable and unstable situations in vertical displacement. 
According to Saffman and Taylor, a characteristic feature of this stage is the tendency of advancing fingers, and the fluid columns between them, to be more-or-less of equal width. At a later stage (Figure 2.3c) the largest finger outgrows all others and spreads laterally to inhibit the growth of its neighbors. Ultimately, this single finger spreads across half the cell width and dominates the flow, a phenomenon called shielding (Homsy, 1987). According to the latter author,

"the pressure in the less mobile phase is harmonic and the interface is nearly isopotential, leading to a larger flux near the tip of any finger that is ahead of neighboring ones."

This suggests that in unstable wetting fronts shielding will not be pronounced because the hydraulic gradient just behind the front is unity at large depth. This is indeed what experiments indicate.

In the above experiments, the viscosity of glycerine exceeded that of air by a factor of 50. When an oil mixture of viscosity $2.75 \mathrm{p}$ (pascal) was used to drive glycerine of viscosity $8 \mathrm{p}$ through a horizontal Hele-Shaw cell, the length of run required for the development of steadily advancing fingers was found by Saffman and Taylor (1958) to be much greater.

Figure 2.4 shows the results of experiments by Saffman and Taylor (1958) in which two oils, Diala and Talpa, which differ in viscosity by a factor of 15 , are displaced horizontally by less viscous water. In the figure, finger width relative to cell width $(r)$ is plotted versus the capillary number $C a=\mu U / T$ where $\mu$ is the viscosity of the oil (the original plot was said by Saffman, 1986, to have been in error). Despite the 15:1 ratio between the viscosities of the two oils, they yield nearly identical $r$ values. This suggests that finger width depends much less (if at all) on the viscosity ratio $M$ than on the capillary number. Note further that $r$ decreases rapidly toward the asymptotic value of $1 / 2$ as $C a$ increases. This however is somewhat misleading. From the theoretical analyses of Saffman and Taylor (1958) and Chuoke et al. (1959), presented in the next chapter, one expects the interface to become unstable as $T$ decreases and $\mathrm{Ca}$ increases beyond some critical values. In fact, Saffman (1959) has shown analytically that the dominant finger becomes unstable in the limit as surface tension disappears. More recent laboratory and numerical experiments have shown that, at very large $\mathrm{Ca}$, finger instability is manifested in chaotic and fractal behaviors; there have been no observations of a compact finger. These findings have brought about a reassessment of the corresponding Saffman-Taylor experiments. Bensimon et al. (1986) stated that Saffman and Taylor had seen the 

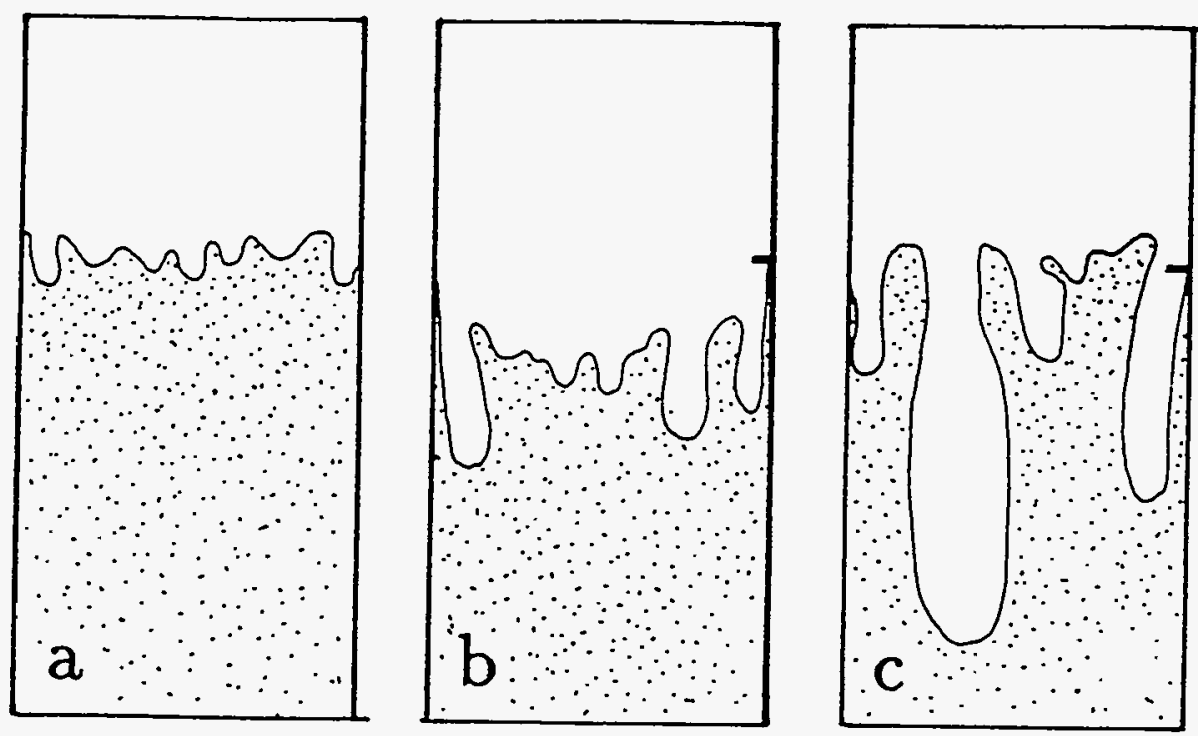

Figure 2.3. Development of instability and fingering as air displaces glycerine downward in a Hele-Shaw cell (modified by Feder, 1988 after Saffman and Taylor, 1958).

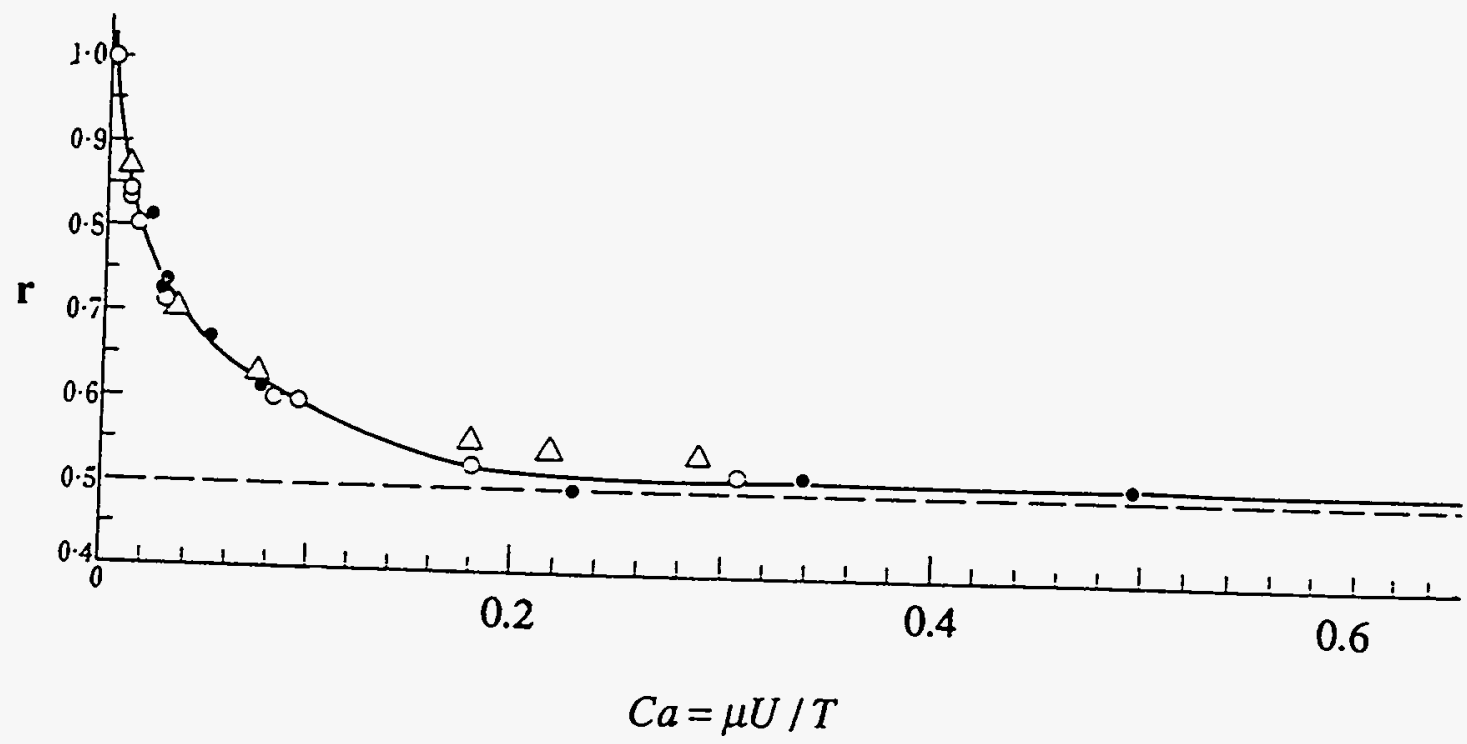

Figure 2.4. Measured values of $r$ for water penetrating into two oils: $\Delta$, Diala (measured from photographs); o, Diala (measured directly); Talpa (measured directly) (after Saffman and Taylor, 1958). 
dominant finger become unstable but had ignored the evidence. Saffman (1986) commented that

"I have no recollection of this observation and cannot find any mention in our paper or correspondence, but the experiments were carried out entirely by Taylor, my contribution being the calculations, and it may be that he saw instabilities and communicated this to others."

Yet Saffman and Taylor (1958, p. 323) did in fact observe that

"as the speed of flow for any given fluid increases, $\mathrm{r}$ rapidly decreases to $r=1 / 2$ and remains close to this value over a large range of speeds, till at high speeds of flow the tongue or finger of the advancing fluid itself breaks down and divides into smaller fingers."

It thus appears that the decrease in $r$ with $C a$ observed by Saffman and Taylor (1958) holds only when $\mathrm{Ca}$ is less than some critical value; when $\mathrm{Ca}$ exceeds this critical value, the dominant finger becomes unstable and has no unique width. As will become clear when we discuss related experiments by Park and Homsy (1985) and Tabeling et al. (1987), all values of $C a$ in Figure 2.4 are less than critical.

In experiments conducted by Chuoke et al. (1959), the Hele-Shaw cell was tilted about $45^{\circ}$ from the horizontal. A glycerine-water mixture displaced a more viscous but less dense oil upward. As anticipated on the basis of Figure (2.2c), the interface remained stable at velocities below a critical value but became unstable at higher velocities. In three experiments conducted at supercritical velocities, the number of fingers increased and their width decreased with an increase in velocity. Fingers in the less viscous displacing water-glycerine solution were wider than the intervening columns of displaced oil; the opposite was noted by Saffman and Taylor (1958). Chuoke et al. did not mention the shielding effect reported by Saffman and Taylor and their results seem not to show it; instead of one dominant finger there are several (Figure 2.5). The widths of these fingers are in good agreement with theoretical predictions; they increase with surface tension and decrease with viscosity ratio.

Perkins and Johnston (1969) ran experiments in a larger Hele-Shaw cell (18 x $40 \mathrm{x}$ $0.031 \mathrm{in}^{3}$ ) at the very unfavorable viscosity ratio of 146 and a relative high velocity. Numerous fingers formed which however tended to coalesce as displacement progressed. In some experiments this resulted in a single dominant finger, in others there were several such fingers. Figure 2.6 illustrates the phenomenon of finger splitting which was later studied more extensively by Park and Homsy (1985), Maxworthy (1987) and others. 

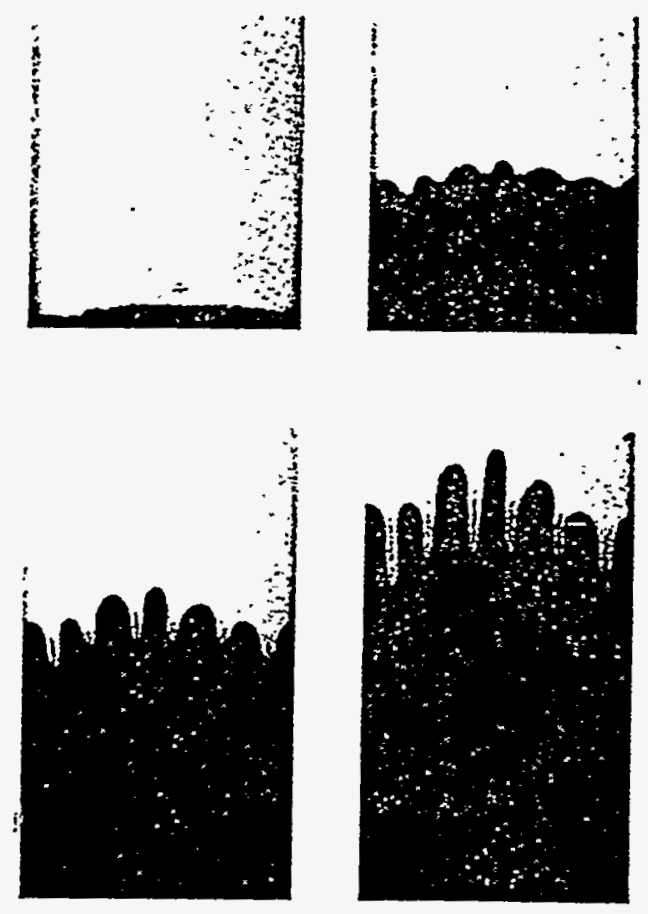

Figure 2.5. Instability of the interface between a water-glycerine mixture and oil in an inclined Hele-Shaw cell (after Chuoke et al., 1959).

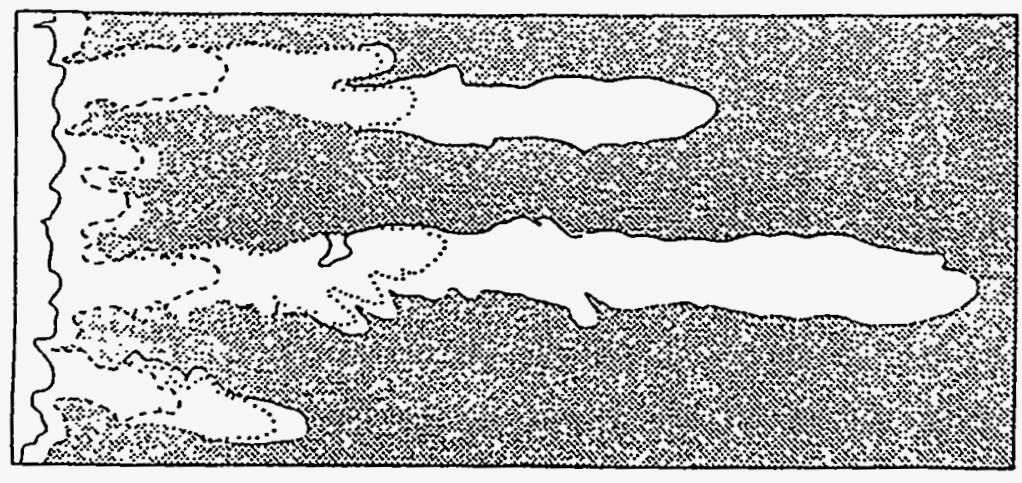

Figure 2.6. Fingering pattern in a Hele-Shaw cell at different stages of displacement with $M=146$ (after Perkins and Johnston, 1969). 
Contradictory results concerning the number of dominant fingers have also been generated by others. Gupta et al. (1973), Gupta and Greenkorn (1974), and Pitts (1980) reported the occurrence of numerous incipient fingers degenerating into a single finger as displacement progressed. White et al. (1976) observed the development of several dominant fingers which never merged. It appears that when the viscosity ratio $M$ is close to one, there is a tendency for several dominant fingers to develop (Maher, 1985). The same happens when the Darcy-Rayleigh number is relatively large (Maxworthy, 1987). The observation that a single dominant finger developed in cells with a relatively large ratio of length to width (8 for Saffman and Taylor, 1958, 17 for Gupta et al., 1973) while several dominant fingers developed in cells with a smaller ratio (3 for White et al., 1976, 3 for Perkins and Johnston, 1969) suggests that this ratio affects the number of dominant fingers. Indeed, White et al. (1976) found the wavelength of fingers in their early stages of growth to be in very good agreement with the "most unstable" wavelength predicted theoretically by Philip (1975). Pitts (1980) found that the shapes of the dominant fingers at finite capillary numbers form a self-similar family.

Gupta et al. (1973) discovered that the growth rate of a dominant finger is linear with time. It is interesting to contrast this with a plume of solute which, in a Fickian regime, spreads at a rate proportional to the square root of time. Park et al. (1984) used a discrete Fourier transform approach to determine the amplitudes, $A_{n}(t)$, corresponding to wave numbers (frequencies) $n$ of the interface at times $t$. A semilog plot of $A_{n}(t)$ versus $t$ for several wave numbers is shown in Figure 2.7. The growth rate is seen to be a function of wave number and time. At low wave numbers and early time the data tend to fall moreor-less on straight lines, suggesting a near-exponential growth with time. The fastest growth rate corresponds to $n=3.59$ in agreement with the linear instability theory of Saffman and Taylor (1958). At later time some of the data corresponding to low wave numbers in Figure 2.7a deviate from the initial straight line, indicating a change in growth rate. Modes associated with higher wave numbers in Figure $2.7 \mathrm{~b}$ exhibit periods of growth and decay, suggesting that they may be neutrally stable.

Park and Homsy (1985) showed experimentally that the critical modified capillary number, $\mathrm{Ca}^{\prime}$, is about 100 for horizontal displacement in a Hele-Shaw cell. Fingers are stable when $C a^{\prime}<100$ but exhibit a periodic growth when $\mathrm{Ca}^{\prime}>100$. As shown in Figure 2.8, the predominant finger becomes unstable and bifurcates when its relative width $r$ approaches 0.5 . One of the two segments outgrows the other and spreads laterally to become the dominant finger. Then it splits again, the process repeating itself 

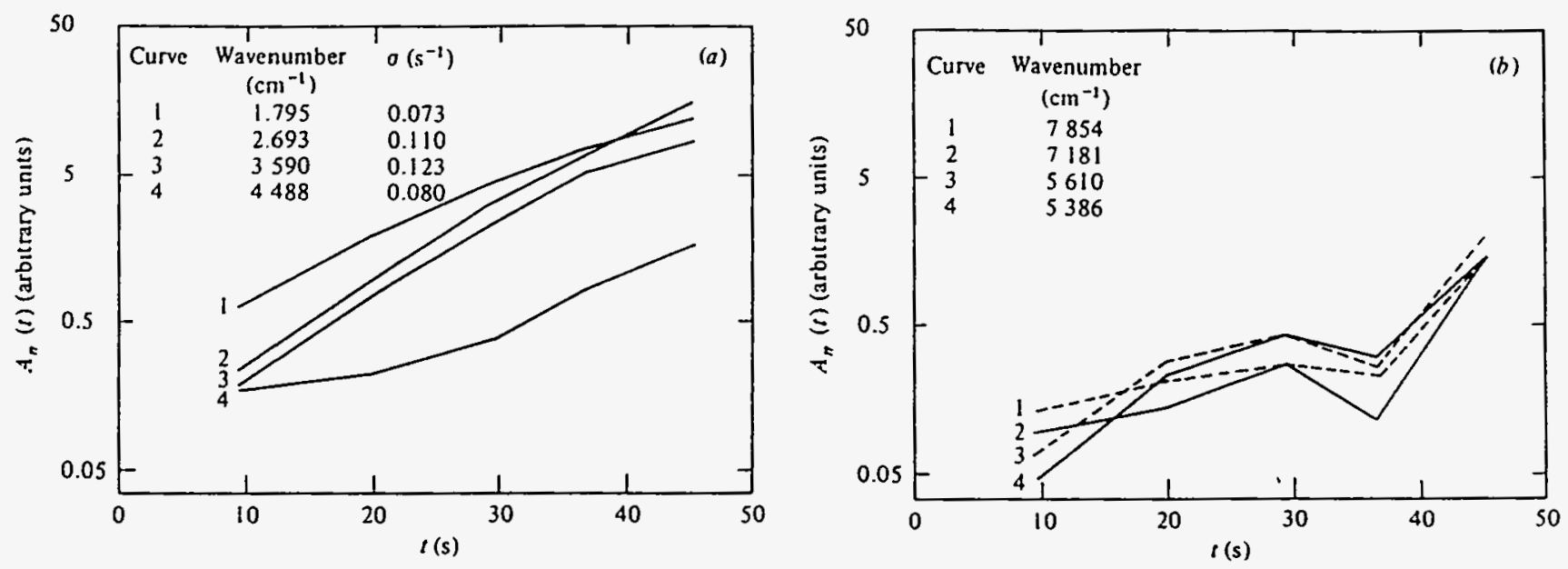

Figure 2.7. Growing and neutral modes of an instability: (a) growing modes; (b) neutral modes (after Park et al., 1984).

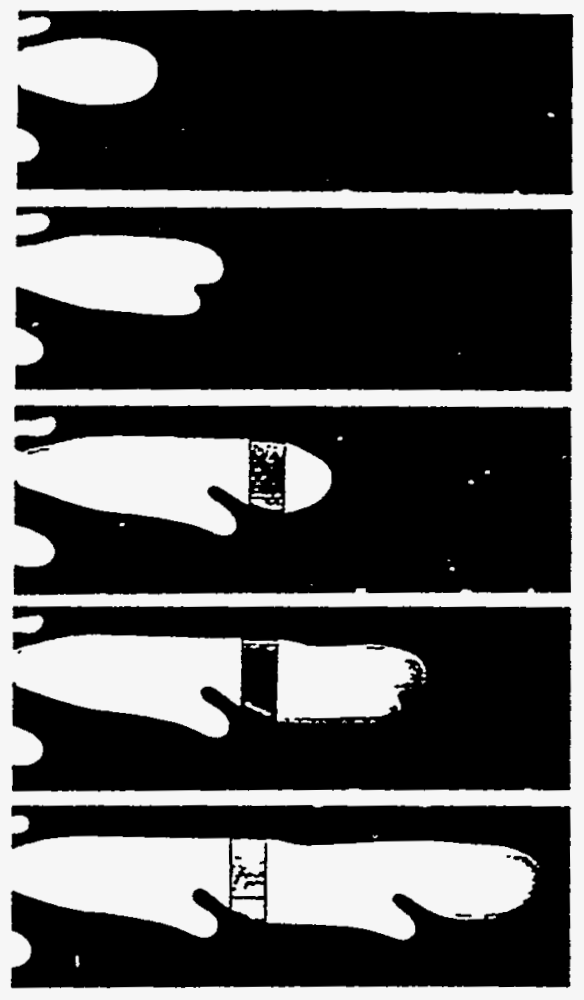

Figure 2.8. Sequence of growth stages for fingering with tip splitting. Time are 90, 110, 140, 171, and 210 seconds (after Park and Homsy, 1985). 
periodically. The explanation is that as a finger widens to become dominant due to the shielding effect, surface tension along its front decreases and the front becomes unstable. This results in bifurcation and the development of narrower fingers which are stable due to increased surface tension. The shielding effect favors one of these two fingers which therefore widens, becomes unstable, and so on. The cycle of shielding, spreading and splitting repeats itself periodically. As $C a^{\prime}$ in the Saffman-Taylor experiment of Figure 2.4 does not exceed 50, it is subcritical and splitting does not occur.

In experiments conducted by Maxworthy (1987) where air displaces silicone oil upward at the extremely large modified capillary number of $C a^{\prime}=5 \times 10^{4}$, the fingers were so unstable as to exhibit secondary splitting into branches and thus formed a highly contorted interface (Figure 2.9). The author found that this interface had formed a random fractal having a dimension of about 1.4 at scales larger than the instability wavelength. Nittmann et al. (1985) calculated the fractal dimension of the interface between two nonNewtonian fluids having the same value of $C a^{\prime}$ to be 1.36 .

Hundreds of experiments concerning the effect of capillary number on the stability of long fingers were conducted by Tabeling et al. (1987) in the broad range of $\mathrm{Ca}^{\prime}$ from $\mathrm{I}$ to more than 7000. For subcritical $\mathrm{Ca}^{\prime}$, finger development was similar to that observed by Saffman and Taylor (1958), with relative finger width decreasing monotonically toward an asymptote as $\mathrm{Ca}^{\prime}$ increases. The authors further found that the number of initial modes had increased more or less as the square root of the velocity. The shapes of the finger tips were semicircular at large capillary number $\left(\mathrm{Ca}^{\prime}>500\right)$ but sharpened as $C a^{\prime}$ decreased. At supercritical $C \mathrm{a}^{\prime}$, the fingers became unstable and split. The critical value of $C a^{2}$ was found to depend strongly on how uniform was the gap between the plates. In a cell having a maximum relative change in aperture $\Delta b / b$ equal to $3 \times 10^{-2}$, the critical value of $C a^{\prime}$ was approximately 3000 . In a similar cell with a smaller maximum $\Delta b / b$ of $3 \times 10^{-3}$, the critical value was 7000 . Clearly, the spatial variability of apertures has a strong influence on the appearance and structure of fingers. It also has an effect on their location: whereas in nonuniform cells the splitting occurred at similar locations in repeated runs, in uniform cells these locations varied from one run to another. Nonhomogeneity may help explain why critical values of $C a^{\prime}$ determined by different researchers were not always the same.

Experiments concerning the effects of Darcy-Rayleigh number and viscosity ratio $M$ (or mobility ratio $A$ ) on fingering are few. Maher (1985) experimented with $A$ values 


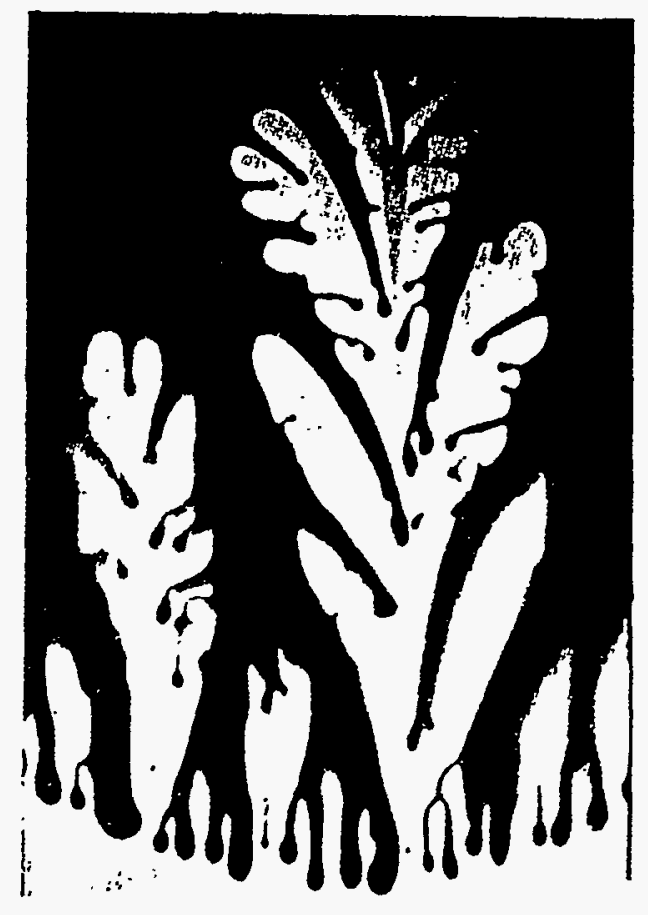

Figure 2.9. Fingering in a vertical Hele-Shaw cell with $\mathrm{Ca}^{\prime}=5 \times 10^{4}$ when air displaces silicone oil upward (after Maxworthy, 1987). 
ranging from 0.0039 to 0.93 . He found that finger development is strongly affected by mobility ratio. As $A$ approaches zero, the viscosities of the two fluids are nearly the same. This has a stabilizing effect and causes a delay in the formation of fingers relative to the case where $A$ is close to 1 . It conforms to an earlier finding by Saffman and Taylor (1958). Maher further established that fingers do not affect the growth of their neighbors when $A \rightarrow 0$ but do when $A \rightarrow 1$. This is consistent with the results of numerical simulations by Tryggvason and Aref (1983). It prevents both shielding and finger splitting when $A \rightarrow 0$, and may help explain differences between the number of dominant fingers observed in various experiments. In Maher's experiments, the longest finger grew as a power of time with an average power of 1.6.

To investigate the effect of gravity, Maxworthy (1987) and Wiggert and Maxworthy (1993) constructed a cell that could be rotated about its horizontal axis. Figure 2.9 has shown the finger pattern that had developed during the upward vertical displacement of silicon oil by air. Figure 2.10 shows what happens when the cell is almost horizontal. Though shielding, spreading, and splitting occur in both cases, in the horizontal cell the initial instability has a much longer wavelength and develops rapidly into a single finger. Secondary instabilities are much less pronounced in the horizontal than in the vertical cell due to the elimination of a destabilizing gravity force.

\subsection{Immiscible Displacement in Porous Media}

Some key experiments on fingering in porous media are listed in Table 2.1. Most of these experiments were conducted on unconsolidated artificial media, two on unconsolidated sand, one on a carbonate rock sample, and one on sandstone. All unconsolidated media contained a narrow range of grain sizes with a relatively high permeability. Most samples were relatively narrow, at times on the order of only a few pore sizes. Hence the experimental results may not be representative of conditions expected in the field.

A major reason for the use of artificial porous media is to allow viewing the development of fingers. In natural materials which are opaque, one may need to use special light penetrating methods (Glass et al., 1989c), x-ray tomography (Hove et al., 1990), the fluid molding method of Pavone (1992), nuclear magnetic resonance (Pearl et al., 1993) or other means to deduce what happens inside.

As in Hele-Shaw cells, numerous incipient fingers develop at the start of the 


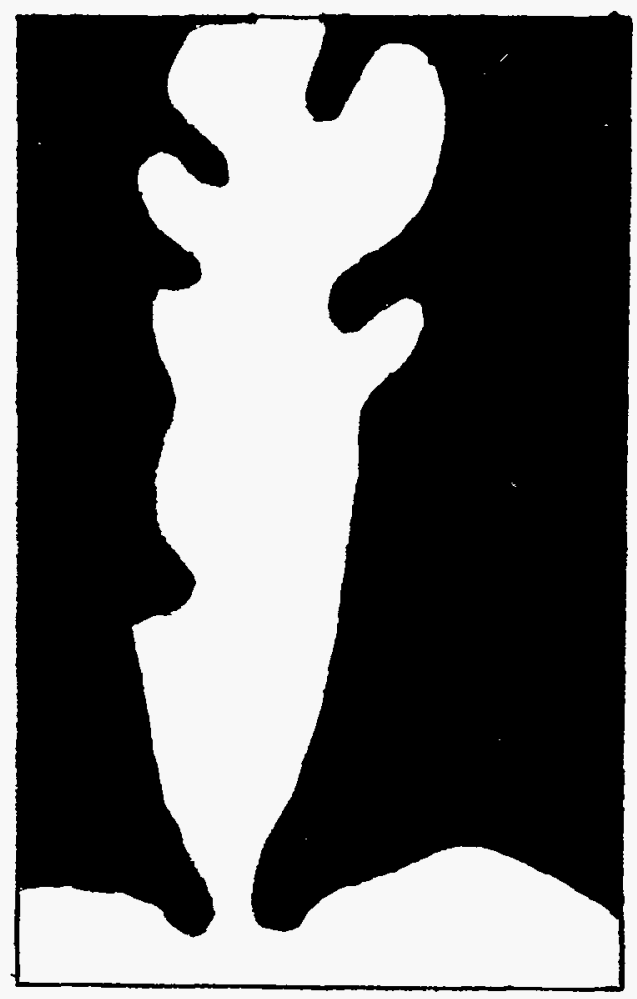

Figure 2.10. Fingering in a horizontal Hele-Shaw cell with $C a^{\prime}=5 \times 10^{4}$ when air displaces silicone oil (after Maxworthy, 1987). 
Table 2.1. Summary of key experiments in porous media.

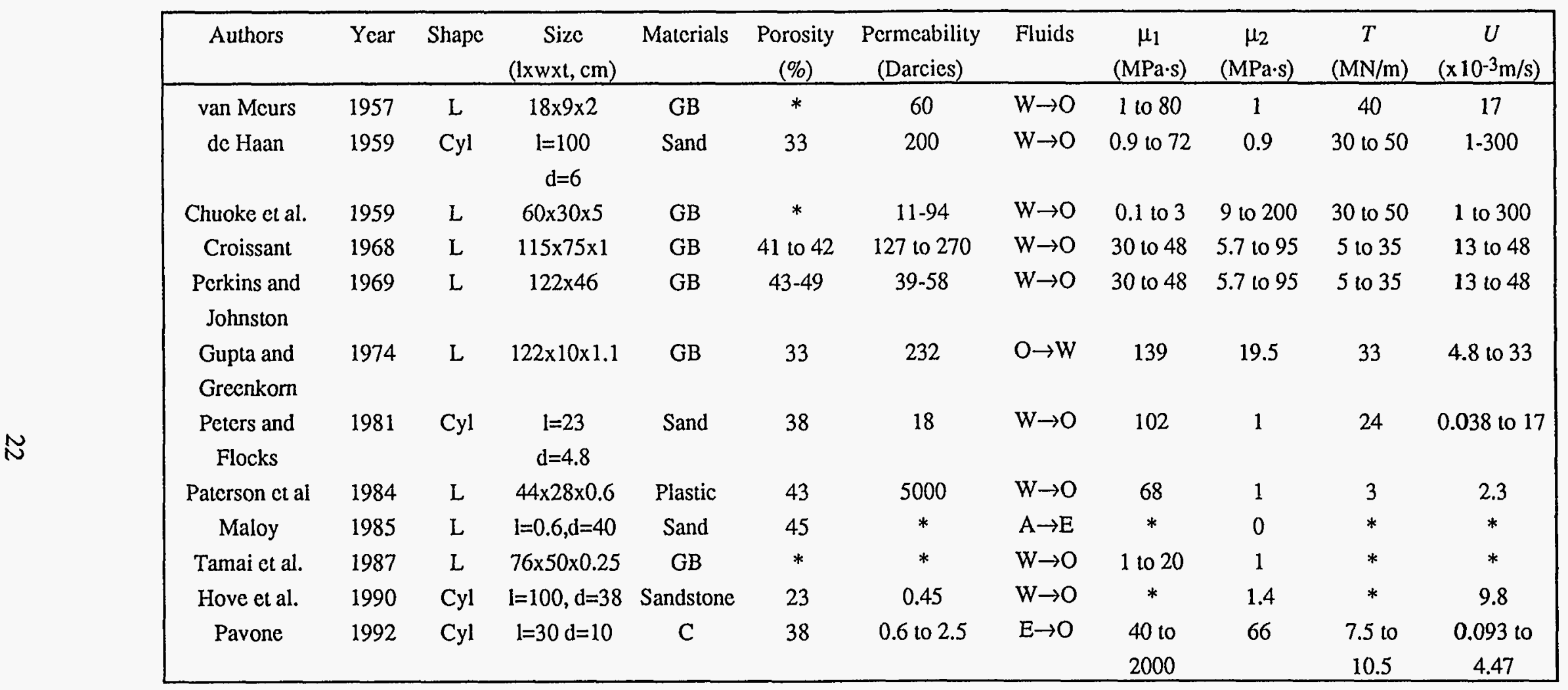

1: length, w: width, t: thickness, d: diameter, $U$ : flow rate, $T$ : surface tension coefficient W: water, O: oil, G: glycerine, A: air, E: epoxy resin

Cyl: cylindrical, L: parallelpiped, GB: glass beads, $\mathrm{C}$ : carbonate rock

*: unknown 
displacement process. These may coalesce either into one dominant finger (van Meurs, 1957; Gupta and Greenkorn, 1974; Croissant, 1968) or into several such fingers (Chuoke et al., 1959; White et al., 1976; Tamai et al, 1987). The tips of dominant fingers may split (Chuoke et al., 1959; Perkins and Johnston, 1969) to form fractal patterns at high capillary numbers (Paterson et al., 1984; Målфy, 1985). Whereas the growth rate of established fingers is linear with time, their width grows in proportion to the square root of time (Croissant, 1968; Perkins and Johnston, 1969; Gupta and Greenkorn, 1974). A high viscosity ratio and/or low bulk interfacial tension favor relatively small fingers (Chuoke et al., 1959). Similar behavior has been observed in Hele-Shaw flows.

Whereas in Hele-Shaw cells the fingers are relatively smooth, in porous media they are distinctly irregular (Figure 2.11). The critical value of modified capillary number for finger splitting in porous media may be much smaller than in Hele-Shaw cells, resulting in dramatically different finger structures under comparable conditions. Figure 2.12 shows patterns observed by Perkins and Johnston (1969) in a cell packed with glass beads at a viscosity ratio of $M=10$. Finger splitting is much more pronounced here than it was in a Hele-Shaw cell at the much larger (less favorable) ratio of $M=146$ (Figure 2.6). Måløy et al. (1985) observed a fractal structure with dimension $D=1.62$ during radial fingering in a random porous medium; an experiment in a Hele-Shaw cell at a comparable capillary number by Paterson (1981) resulted in fingers that are much smoother and wider. These differences result from the pore scale heterogeneity of porous media. The effects of macroscopic medium heterogeneity on finger development have been studied in connection with miscible displacement by Brock and Orr (1991) and Davies et al. (1991). To our knowledge, no similar studies have been conducted on immiscible displacement.

The wetting properties of the fluids were found to have an important effect on fingering. de Haan (1959) found that fingers of water in oil-wet media are on the order of magnitude of the pores and form a fractal pattern. In water-wet media the water phase is much more continuous. Similar observations have been reported by Stokes et al. (1986). Peters and Flock (1981) showed that water fingers are almost eight times wider in a water-wet medium than in an oil-wet medium.

\subsection{Infiltration Into Unsaturated Media}

The stability of air-water interfaces such as wetting fronts which may develop during vertical infiltration into unsaturated porous or fractured media is of special concern in this report. We therefore discuss it separately in this section. During infiltration, the 


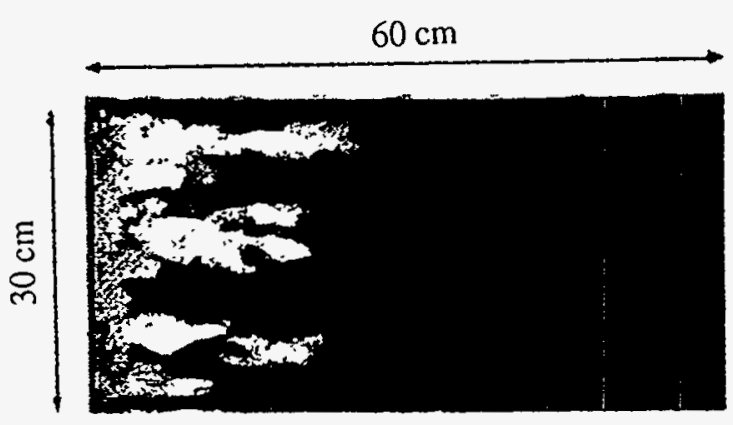

(a)

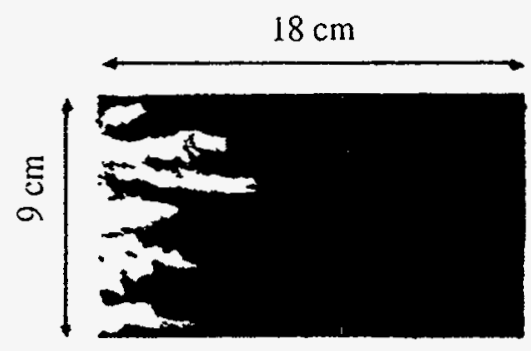

(b)

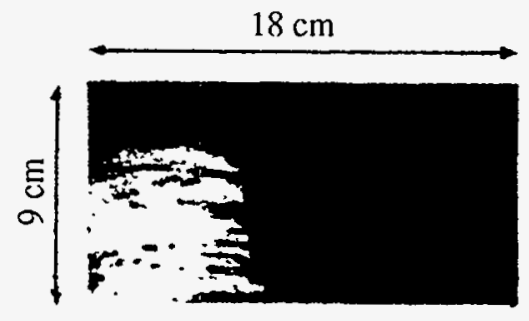

(c)

Figure 2.11. Fingering in porous media: (a) $M=9.45, C a=2 \times 10^{-5}$; (b) $M=$ 70.5, $C a=1.2 \times 10^{-4}$; (c) $M=202, C a=3 \times 10^{-3}$ (after Chuoke et al., 1959).
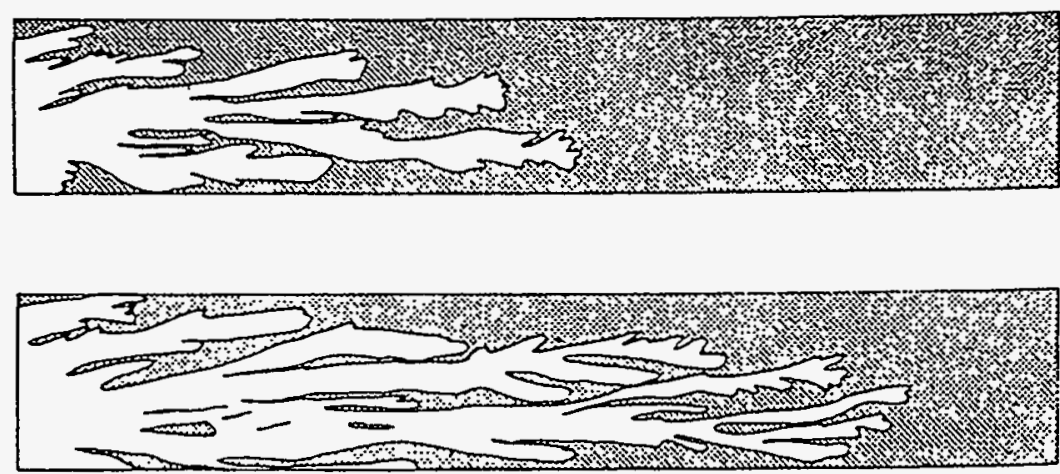

Figure 2.12. Fingering in a two-dimensional bead-packed cell at $M=10$ (after Perkins and Johnston, 1969). 
displacing fluid is water and the displaced fluid is air. This corresponds to case (h) in Figure 2.2 where a more viscous and dense fluid displaces a less viscous and dense fluid downward. Gravity acts as a destabilizing force and viscosity as a stabilizing force. The advance of the wetting front is gravity-driven, and one expects it to be stable at high rates of advance, unstable at low rates. As the viscosity of water $\left(\mu_{2}\right)$ is much greater than that of air $\left(\mu_{1}\right)$, the mobility ratio $A=\left(\mu_{1}-\mu_{2}\right) /\left(\mu_{1}+\mu_{2}\right)$ is close to -1 , and the modified capillary number $C a^{\prime}=\mu_{1} U /(k T)$ is close to zero. As $\rho_{1} \ll \rho_{2}$, the Darcy-Rayleigh number can be approximated by $D R \approx-\rho_{2} g k /\left(\mu_{2} U\right)=-K / U$ where $U$ is the velocity of the wetting front and $K$ is the hydraulic conductivity behind this front. When $U$ and/or $K$ vary with time (as is usually the case during infiltration), so do $D R$ and $C a^{\prime}$.

Deecke (1906) noticed that saturated fingers had formed in wettable dune sand after infiltration of a heavy rain at the Darr peninsula in Germany. This was evidenced by thousands of blunt and tapered wet sand cores left over after the dry sand between them had been removed by a strong wind the following day. van Dieren (1934) discovered preferential flow paths in water repellent dunes on the island of Terschelling in the Netherlands. Similar field observations have been made by Bond (1964) and DeBano (1969) in water repellent soils and Gripp (1961) and Gees and Lyall (1969) in water wettable soils. Most of the fingers ranged from $5 \mathrm{~cm}$ to $25 \mathrm{~cm}$ in diameter. Initially, these were dismissed as anomalies (Hendrickx and Dekker, 1991). Later, the phenomenon was observed experimentally by Tabuchi (1961), Miller and Gardener (1962), Peck (1965), Smith (1967) and others.

Hill and Parlange (1972) were among the first to recognize that the phenomenon is related to wetting front instability. They demonstrated experimentally the existence of wetting front instability and fingering in a system composed of two layers, a finer soil above a coarse one. This has spurred laboratory experiments by numerous workers including White et al. (1977) on the effect of enhanced air pressure ahead of a wetting front, Diment and Watson (1985) on two-layered soils and redistribution after infiltration under various uniform initial moisture contents, Tamai et al. (1987) on redistribution in glass beads, Glass and Steenhuis (1984) and Glass et al. $(1987,1988)$ on two-layered soils under dry and non-uniform initial moisture contents, Glass et al. $(1990,1991)$ on threedimensional infiltration into two-layered soils, Baker and Hillel $(1990,1991)$ on sharp and diffusive wetting fronts under dry and moist conditions in two-layered soils, Hendrickx and Dekker (1991) on uniform non-layered soils under natural precipitation, and Nicholl et al. (1994) on redistribution in fractures. Specially designed field studies 
were conducted in Connecticut by Starr et al. $(1978,1986)$; on eastern Long Island, New York, by Glass et al. (1988); in the Netherlands by van Ommen et al. (1989), Hendrickx et al. (1993) and Ritsema et al. (1993); and by others.

The above experiments have revealed that a wetting front may become unstable if it moves from a fine into a coarse soil layer, if saturated hydraulic conductivity increases with depth, if air ahead of the front is compressed, and if moisture redistribution occurs following infiltration. Among these conditions, layering has been studied most extensively (Hillel, 1987). Among the several thousand soil series in the United States, about 350 belong to family groupings with fine or coarse loamy layers covering sandy or sandy-skeletal textures (Hill and Parlange, 1972). We discuss below under separate subheadings laboratory infiltration into layered media, similar infiltration into nonlayered media, and field experiments.

\subsubsection{Laboratory Infiltration Into Layered Media}

In the laboratory experiments of Hill and Parlange (1972), a layer of finer sand was placed above a layer of coarser sand in a plexiglass chamber. Vents were placed at the base of the chamber to permit air to escape during infiltration. The sands were initially air-dried and sieved. The saturated hydraulic conductivity of the coarse sand was 20 times larger than that of the fine sand. The experiment was started by ponding water quickly and evenly across the top of the fine layer with a pump. The depth of ponding was maintained constant throughout the experiment. The plexiglass chamber permitted visual observation and photographing of the advancing wetting front. At early time, a stable uniform front moved through the top sand layer. When it reached the interface, the wetting front invaded the bottom layer in the form of fingers. From this time on, the total infiltration rate remained virtually constant. The authors noticed that fingers moved downward at a speed close to the ratio between the saturated hydraulic conductivity of the coarse sublayer and its initial water content; finger widths were nearly uniform and independent of flow rate while the number of fingers was directly proportional to flow rate. We will see that this differs from behavior observed later by Glass et al. (1989b). For a reverse sequence of a coarse texture over a fine texture, Hill and Parlange (1972) found the front to move uniformly through the coarse top layer and to remain stable in the fine bottom layer. In related experiments by Samani et al. (1989), the authors established the critical hydraulic conductivity ratio between layers to be 20 . Though the effect of initial moisture content on this critical ratio has not been studied, we expect the latter to increase with the former due to its stabilizing influence, as will soon become evident. 
To visualize the process more clearly, Glass et al. (1989c) developed a technique based on the fact that light transmission through sand increases with an increase in moisture content. Their experiments were conducted by placing a $10 \mathrm{~cm}$ layer of fine sand on top of an $80 \mathrm{~cm}$ layer of coarse sand between closely spaced glass walls. The sands were initially washed, sieved, and air-dried. A diffuse source of high-output fluorescent light was placed on one side of the sand-filled chamber. Water was then applied uniformly at the top surface of the fine sand and allowed to pond $1.5 \mathrm{~cm}$ above it while air was allowed to escape through the bottom. The wetting process was visible to the eye from the other side of the chamber and was recorded on videotape. Some of the experimental results are illustrated in Figure 2.13 where red corresponds to relatively high moisture content and black to lower moisture content. Frames 3 - 10 show moisture patterns in the coarse sand, due to ponded infiltration through the overlying fine sand, at various times during the first experimental cycle. Frames $12-16$ show a subsequent cycle initiated after 24 hours of moisture redistribution due to gravity drainage. Flow in the top layer is characterized by a flat wetting front and vertical streamlines. Immediately behind the interface between the sands, the front breaks into more-or-less regularly spaced fingers about $2-3 \mathrm{~cm}$ wide. Some of these incipient fingers merge into lager fingers which tend to advance at a faster pace. The fingers persist and form core areas that conduct most of the flow through the bottom sand layer. During this initial stage in the evolution of unstable flow, the fingers have near uniform widths and look like hanging ropes. Their shapes are distinctly different from those encountered in experiments described earlier. The next stage involves slow. lateral movement of moisture from the fingers into the dry sand between them, as seen in frames 9 - 10. Finger widths seem to be only marginally affected by this lateral moisture redistribution, which appears to cease once the entire volume between the fingers has been wetted. The lateral moisture pattern and vertical flow rate stabilize; the latter is controlled by the more restrictive top layer and takes place primarily through the fingers.

As seen in frame 12 of Figure 2.13, water contents at the start of Cycle 2 are slightly higher along the antecedent fingers than elsewhere. In frames $13-16$, these residual fingers are seen to evolve into new ones. They propagate at a somewhat more uniform but slower rate than during Cycle 1. However, lateral moisture movement is now faster and the profile stabilizes more rapidly.

An interesting feature revealed by Figure 2.13 is that maximum saturation occurs just above the tip of each finger. This was explained theoretically by Selker et al. 

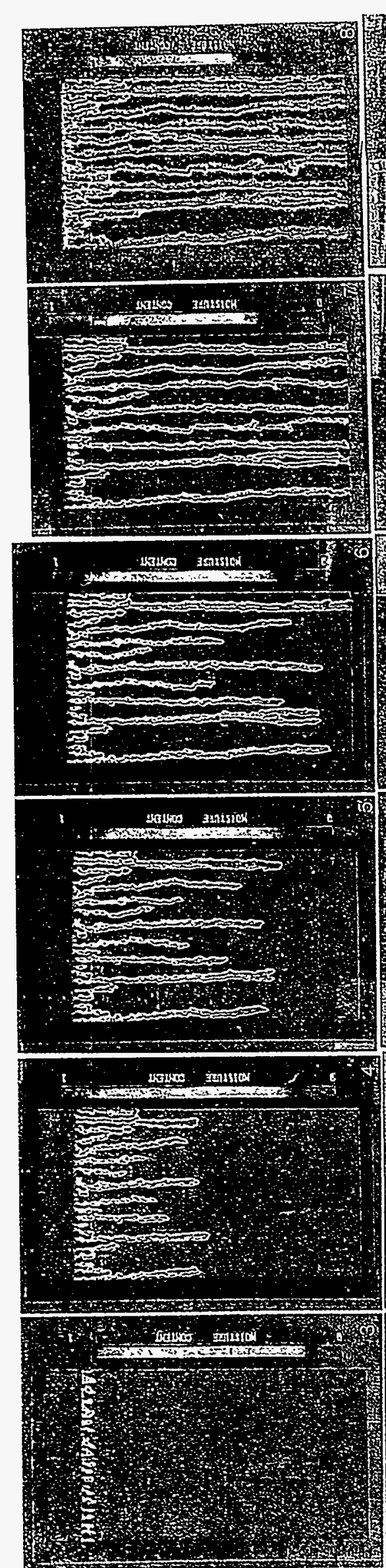
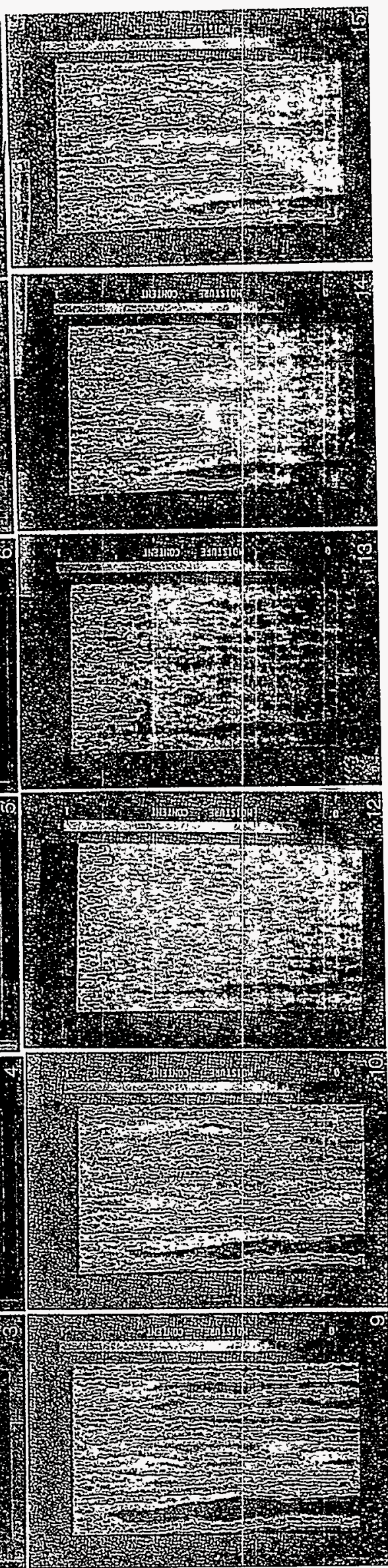
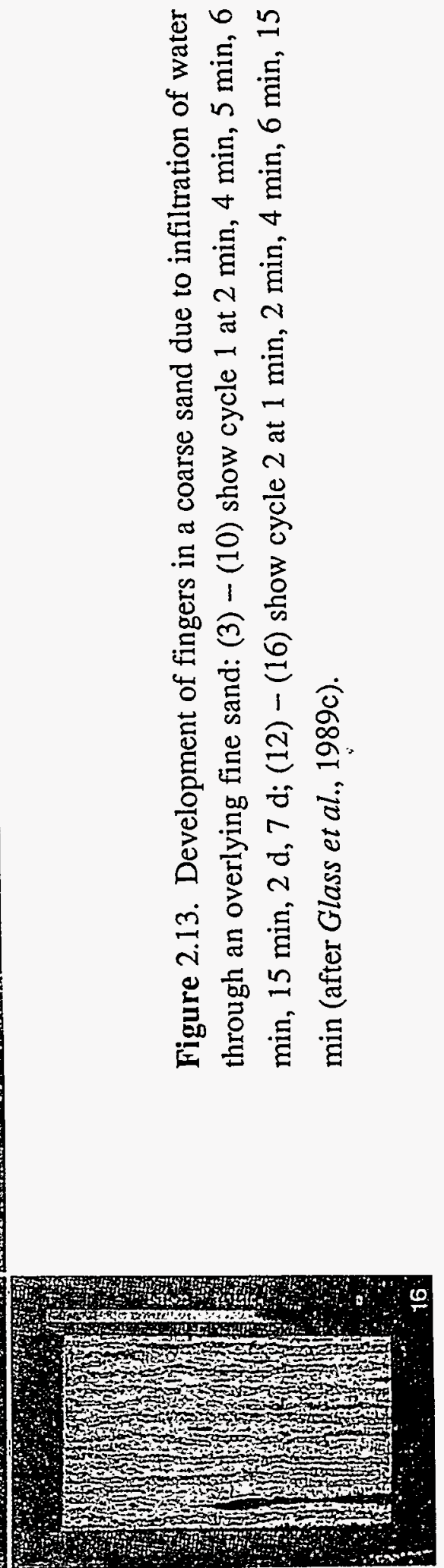
(1992a,b) based on Richards' equation under the assumption of constant tip velocity, which is supported by experiments as illustrated schematically in Figure 2.14.

In another similar experiment, Glass et al. (1989b) visualized the infiltration process by adding a nonsorbing dye (USDA Red no. 3 ) to the water (without using a light source). Here too they found that infiltration at the surface is controlled by the finer top layer and attains a constant rate once the wetting front crosses the interface between the sand layers. This is consistent with earlier observations by Hill and Parlange (1972). On the other hand, the former authors found that finger widths and growth rates increase with infiltration rate in the manner illustrated in Figure 2.15; this is not consistent with what the latter authors had observed. Glass et al. attributed the discrepancy to soil heterogeneity in the experiments of Hill and Parlange, as evidenced by uneven finger sides.

Glass et al. (1990) conducted three-dimensional experiments in a cylindrical chamber $30 \mathrm{~cm}$ in diameter. The cylinder consisted of twelve rings, $5 \mathrm{~cm}$ long, separated by gaps to allow the escape of air. Four two-layered experiments were conducted with different top layers. Water with a low concentration of USDA Red no. 3 was allowed to pond on top of the fine top layer to a depth of $1.5 \mathrm{~cm}$. The underlying coarse sand column was split after each experiment to expose finger cross-sections at selected depths (Figure 2.16). The fingers are seen to be distributed more or less uniformly at all depths. Jointly, the four experiments suggest that an increase in flow rate causes an increase in finger diameter and growth rate, but has little effect on the numbers of fingers.

Finger splitting has been noted during infiltration but is much less pronounced than we saw earlier in Figure 2.9. Since capillary number increases with propagation rate, so does finger splitting become more pronounced. Recently, Chang et al. (1994) found two unstable wetting fronts to form fractals with dimensions 1.21 and 1.31 , respectively.

An intuitive explanation of wetting front instability in two-layered media was offered by Hill and Parlange (1972). They saw the phenomenon as being somewhat analogous to that of flow through a slab of fine saturated porous material suspended in air. Prior to exiting at the bottom, the water accumulates in the form of discrete droplets which eventually acquire sufficient weight to overcome surface tension, detach themselves from the slab, and fall freely though the air. When the air is replaced by a coarse porous material, water still accumulates at discrete points along the interface but its downward movement is now retarded by much greater viscous resistance and capillary effects. This results in the development of viscous fingers. 


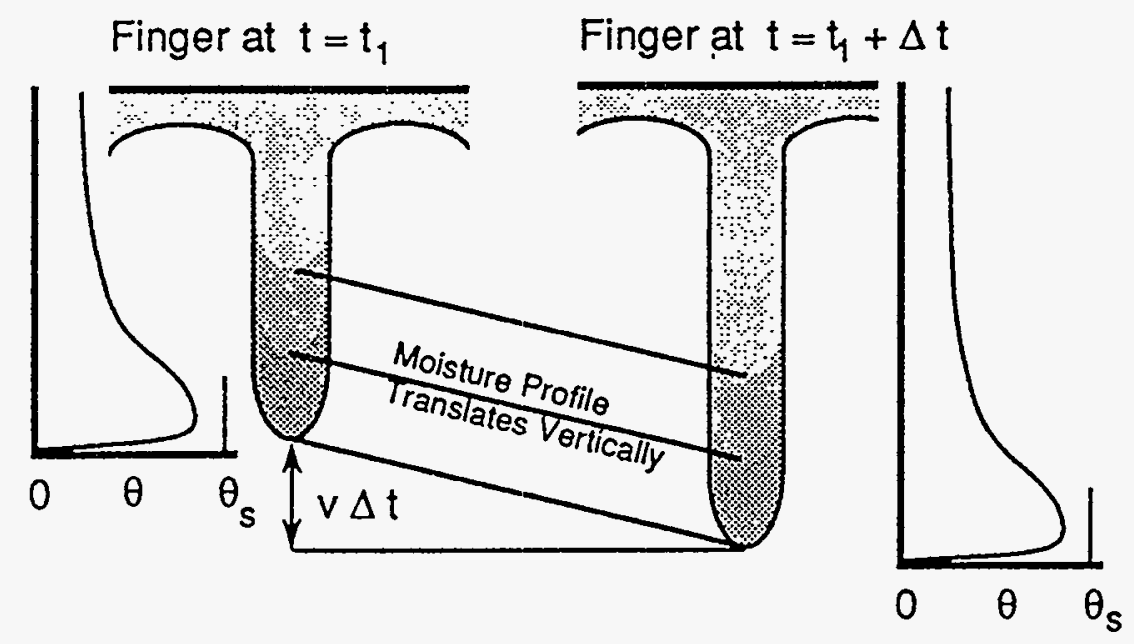

Figure 2.14. Sketch of observed finger translation (after Selker et al. 1992b).

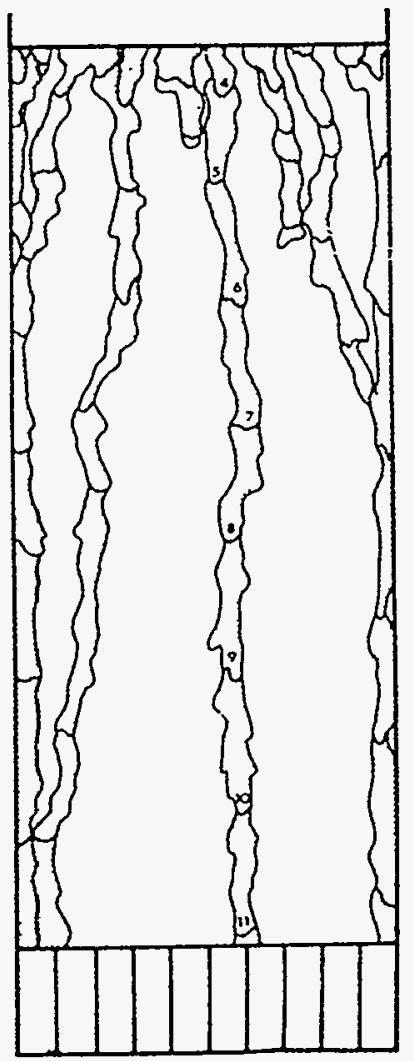

(a)

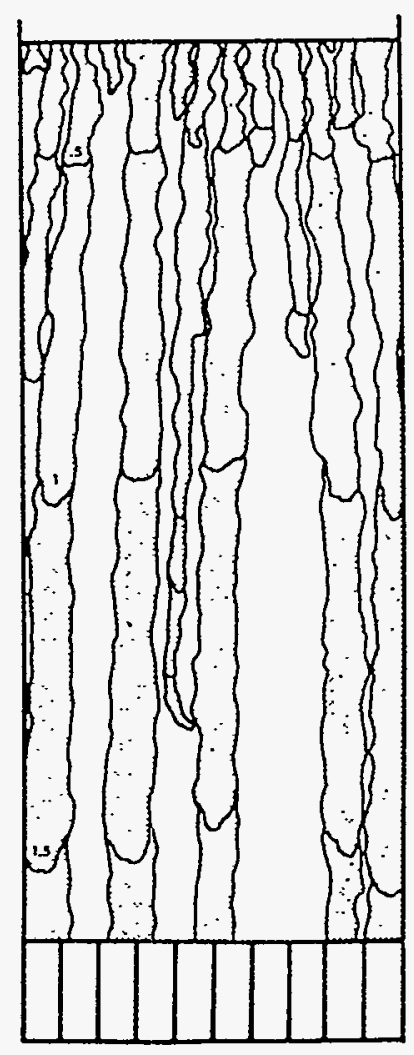

(b)

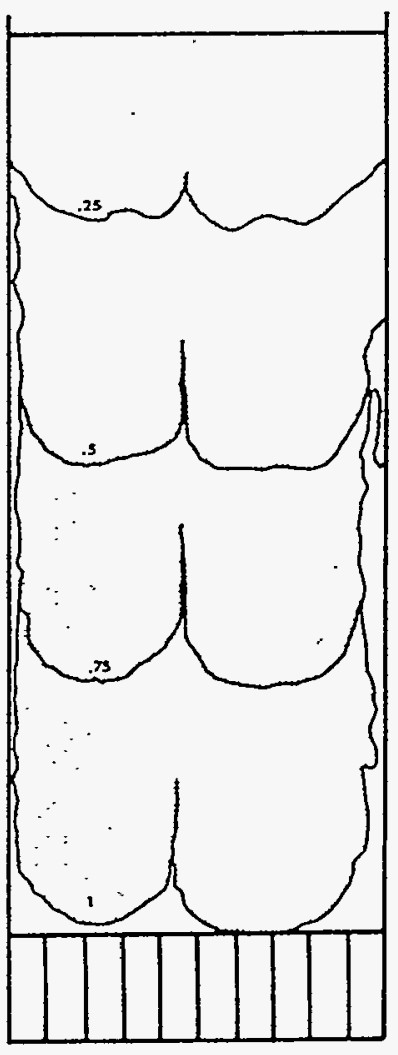

(c)

Figure 2.15. Effect of infiltration rate on finger width and propagation rate: (a) low rate; (b) intermediate rate; (c) high rate (after Glass et al., 1989b). 

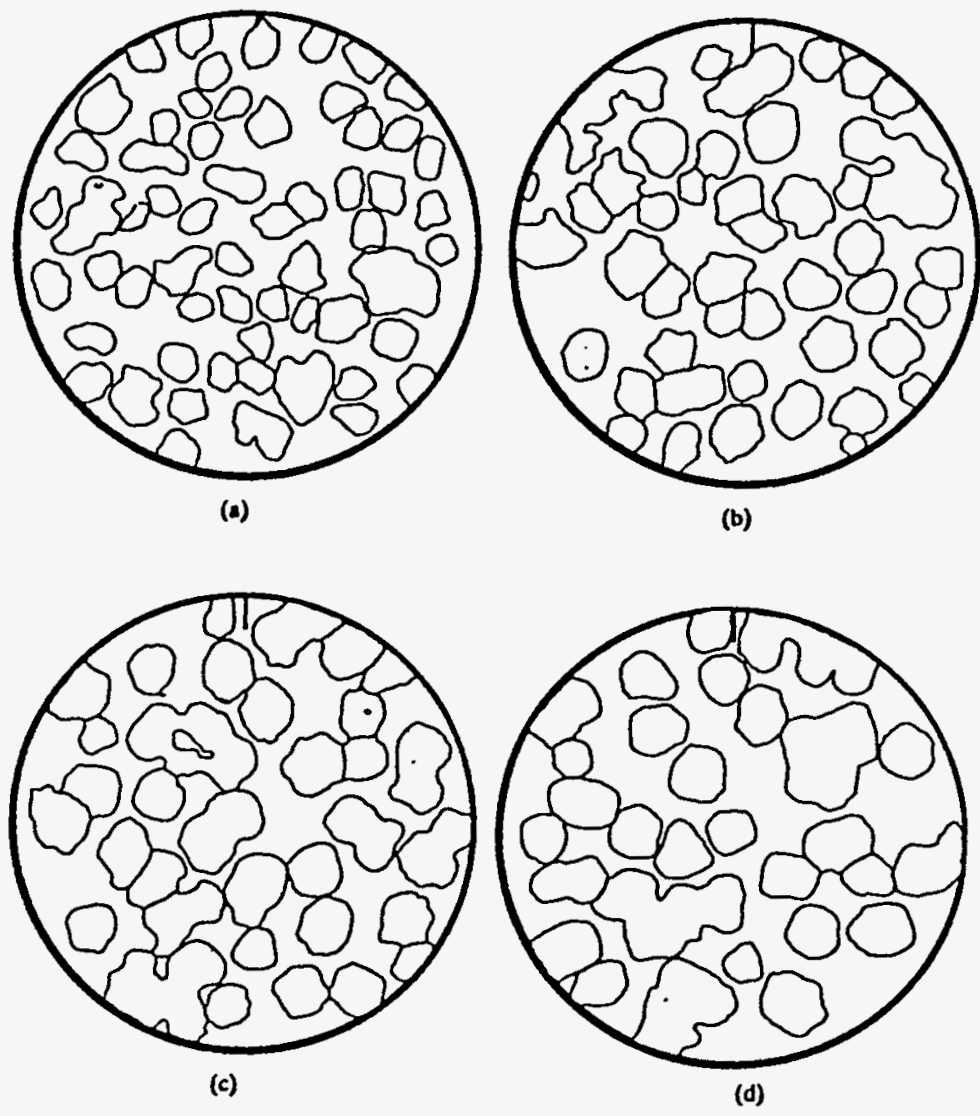

Figure 2.16. Cross-sections showing fingers in three dimensional experiments. Depth from textural interface is (a) $10 \mathrm{~cm}$, (b) $20 \mathrm{~cm}$, (c) $30 \mathrm{~cm}$, and (d) $40 \mathrm{~cm}$ (after Glass et al., 1990). 
A more satisfying explanation for the same phenomenon was suggested by Hillel and Baker (1988). They pointed out that, under a uniform vertical hydraulic gradient (of unit magnitude in pure gravity flow), mass continuity requires that the area available for flow be smaller in the more permeable layer. The system adjusts to this requirement by concentrating the flow along discrete channels or fingers. We will discuss their theory in more detail later.

Since natural geological media are seldom dry, it is important to understand the effect of initial moisture content on wetting front instability. Smith (1967) noted that fingers moving down through a dry sand had widened upon reaching a wetter region. A similar observation was made by Liu et al. (1991), as illustrated in Figure 2.17. Diment and Watson (1985) found that even slightly elevated initial water contents in the coarser bottom layer of a two-layer system may have an important stabilizing effect on the wetting front. On the other hand, field experiments by $\operatorname{Starr}(1978,1986)$ have revealed fingers in soils that had a high initial water content. Glass et al. (1988) attributed the apparent lack of evidence for fingering in laboratory soils at uniformly high initial water content to lateral flow which causes fingers to widen and merge. We saw earlier that in soils wetted nonuniformly by antecedent fingers, new ones may form at the same locations. If the uniform layer of coarser sand at the bottom is dried between wetting events by blowing hot air through it, new fingers form at random locations (Baker and Hillel, 1991).

The sharper is a wetting front, the less stable it tends to be. White et al. (1976) found experimentally that conditions which cause instability in a coarse sand may give rise to a stable front in fine sand. The authors attributed this to the diffuse nature of the front in fine sand. Similar experimental results, supported by theory, were reported by Diment et al. (1982) and Diment and Watson (1983, 1985). Baker and Hillel (1991) varied grain size and moisture content in the bottom coarse layer to demonstrate that hydraulic diffusivity and initial saturation have a mutually reinforcing effect. Acting together, these factors cause fingers to be wider and less distinct than would be the case if only one factor was present.

We mentioned earlier that Glass et al. (1989b) had attributed differences between their experimental results and those of Hill and Parlange (1972) to soil heterogeneity. Figure 2.18 shows fingers advancing into a coarse sand during a preliminary two-layer experiment by Glass et al. (1989b). The irregular fingers were attributed by the authors to nonuniform placement of the sand by means of a funnel. In an experiment by Schwille 


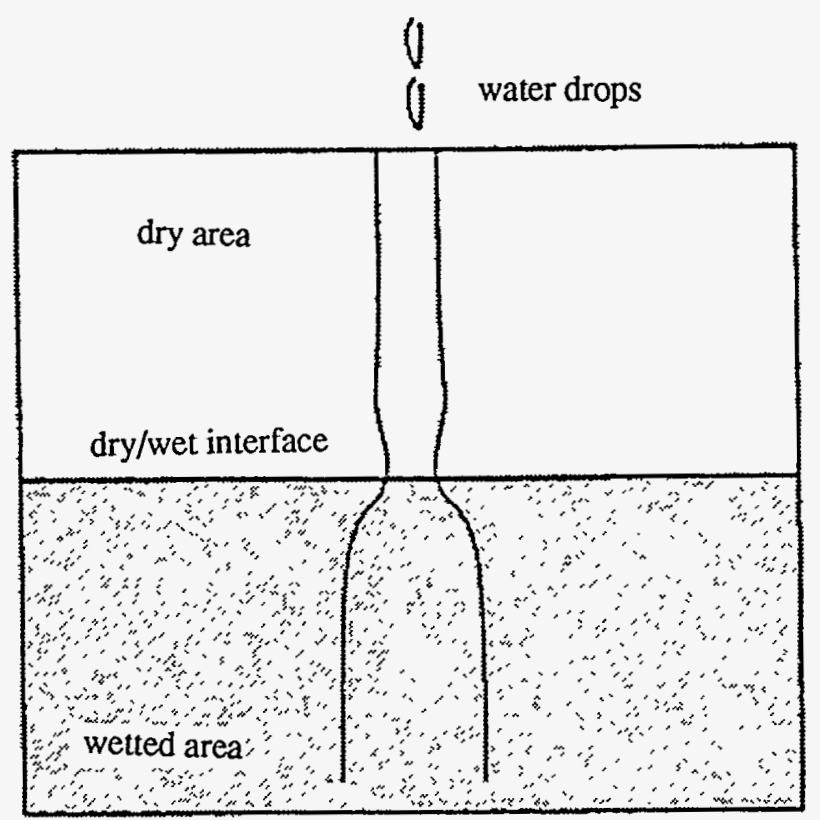

Figure 2.17. Finger movement through a dry sand into a wetted sand (after Liu et al., 1991).
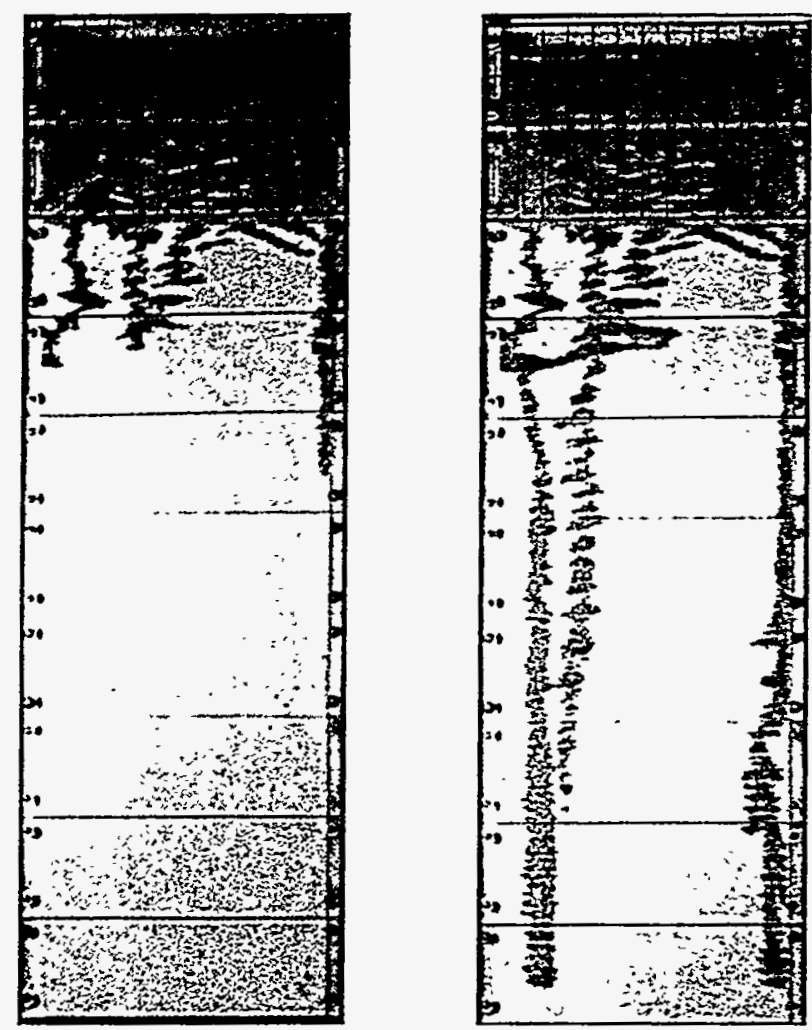

Figure 2.18. Preliminary experiment demonstrating the effects of slight heterogeneities on finger meandering and merger in a two-layer soil (after Glass et al., 1989b). 
(1988), a $200 \mathrm{~cm}$ thick profile of alluvial sediments from a gravel pit was removed layer by layer and reconstructed in a glass column $200 \mathrm{~cm}$ high and $40 \mathrm{~cm}$ in diameter. The profile comprised primarily intermediate to coarse sands with some fine gravels. The depth interval between $90 \mathrm{~cm}$ and $100 \mathrm{~cm}$ contained a large portion of fine sand. Each day, a $10 \mathrm{~mm}$ layer of water with fluorescent dye was applied at the top of the column. The movement of the dyed front was studied with an ultraviolet lamp. Its positions at successive times are depicted in Figure 2.19 and are seen to be quite irregular, forming first one and then two broad fingers. The irregularity of the fingers suggests that heterogeneity might have played an important role in their formation.

\subsubsection{Laboratory Infiltration Into Nonlayered Media}

There have been few laboratory studies on wetting front instability in nonlayered soils. Peck (1965) observed the phenomenon to occur during infiltration into a dry, uniformly compacted sand column. Water at a constant pressure was applied at the top and the air phase was confined so that its pressure increased as the wetting front advanced downward. The author observed a "water tongue" of length $37 \mathrm{~cm}$ as the mean wetting front reached a depth of $45 \mathrm{~cm}$ but did not pay particular attention to it. The phenomenon was later verified in specially designed Hele-Shaw experiments by White et al. (1977). When the air ahead of the wetting front was permitted to escape, the front appeared stable. When air escape was blocked, distinct fingers were observed to grow with time.

White et al. (1977) also studied wetting front instability during infiltration into a Hele-Shaw cell whose aperture varied linearly with depth, remaining constant in the horizontal direction. This is analogous to a quadratic variation in permeability with depth. Water level at the top was maintained constant in each experiment but varied from one experiment to another. The wetting front was always stable when the permeability decreased with depth, but unstable when the permeability increased with depth.

Diment and Watson (1985) and Tamai et al. (1987) found instability to occur in uniform soils as soon as all ponded water entered the medium and redistribution commenced. Figure 2.20 is an example of what may happen after very brief periods of uniform infiltration into an initially dry uniform sand. Nicholl et al. (1994) observed a similar phenomenon in a natural fracture from the Bandelier tuff near Los Alamos, New Mexico. Before infiltration, a clamping fixture was used to hold the fracture surfaces in close contact and vertical. The upper boundary of the fracture was covered with silicon

putty to form a narrow reservoir. Approximately $0.4 \mathrm{~cm} 3 / \mathrm{cm}$ water with dye was applied 


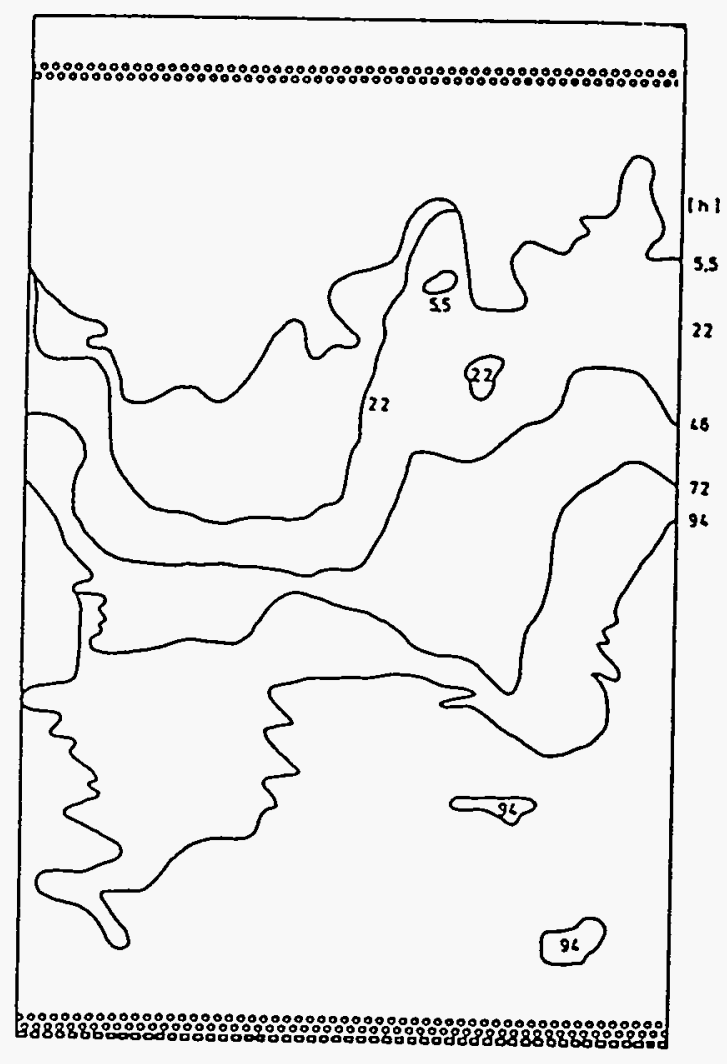

Figure 2.19. Development of dyed infiltration front in a heterogeneous medium (after Schwille, 1988) 
rapidly to the reservoir. At the conclusion of the experiment, the fracture was disassembled to allow examination of its wetted surfaces. As shown in Figure 2.21, the infiltration front appears to have wetted uniformly the upper $20 \mathrm{~cm}$ of the fracture, implying a stable flow regime at this shallow depth. Below this depth the front seems to have broken into near-vertical fingers. Nicholl et al. also conducted experiments in an artificial, transparent roughed-walled fracture. The experiments were done at various angles $\delta$ between the dip of the fracture and the vertical (a vector pointing in a direction opposite to that of gravity), under various depths of ponding, $L_{a}$. Figure 2.22 illustrates the case where $\delta=139^{\circ}$ and $L_{\alpha}=9.43 \mathrm{~cm}$. During infiltration the front was relatively stable (Figure 2.22a). Instability set in after the cessation of ponded infiltration (Figure 2.22b). Thereafter, fingers developed rapidly. The largest and fastest fingers carried most of the available fluid at their tips; shorter fingers eventually stopped growing (Figure $2.22 \mathrm{c}$ and d). Figure 2.23 shows that the larger was the depth of applied water, $L_{a}$, the fewer and wider were the fingers and the faster they propagated. Figure 2.24 demonstrates that an increase in $\delta$ causes an increase in propagation rate due to gravity, but little change in the number of fingers or their widths.

Hendrickx and Dekker (1991) observed finger development during rain infiltration into both wettable and water-repellent soil columns packed in two concrete lysimeters, respectively. Following a total precipitation of $40.3 \mathrm{~cm}$ over a period of 120 days, the soil was excavated to visualize flow patterns. The results were inconclusive as to the potential impact of hydrofobicity on fingering. More will be said about this in connection with field experiments below.

Selker et al. (1992a) found the wetting front in a uniform sand to become unstable when water was applied uniformly at a constant rate equal to about $10 \%$ of the saturated hydraulic conductivity. Nicholl et al. (1994) found that, more generally, such an instability occurs in porous media and fractures whenever the rate of infiltration is less than the saturated hydraulic conductivity. The pattern of fingers under these conditions is closely reminiscent of that under other conditions.

\subsubsection{Field Experiments}

A number of field experiments have revealed the formation of fingers during water infiltration into unsaturated soils. Some of these are listed in Table 2.2 and discussed below. 


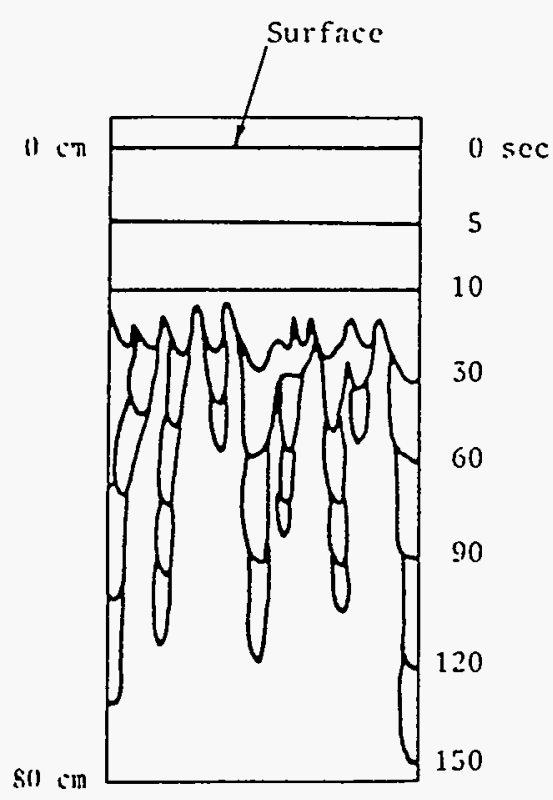

(a)

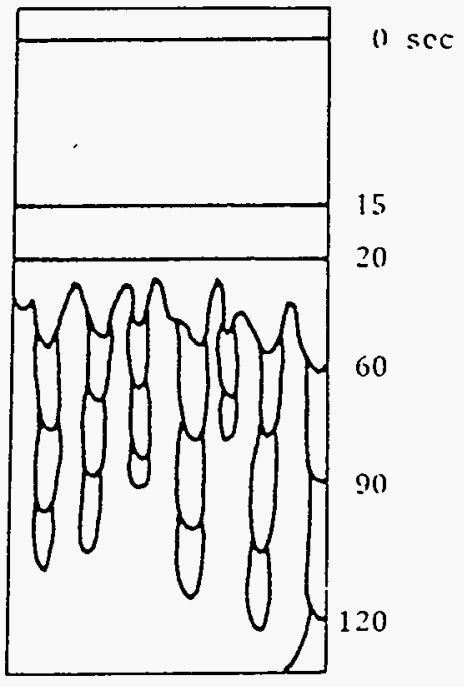

(b)

Figure 2.20. Fingering during redistribution in dry sand following infiltration time of (a) 10 seconds, (b) 20 seconds (after Diment and Watson, 1985).

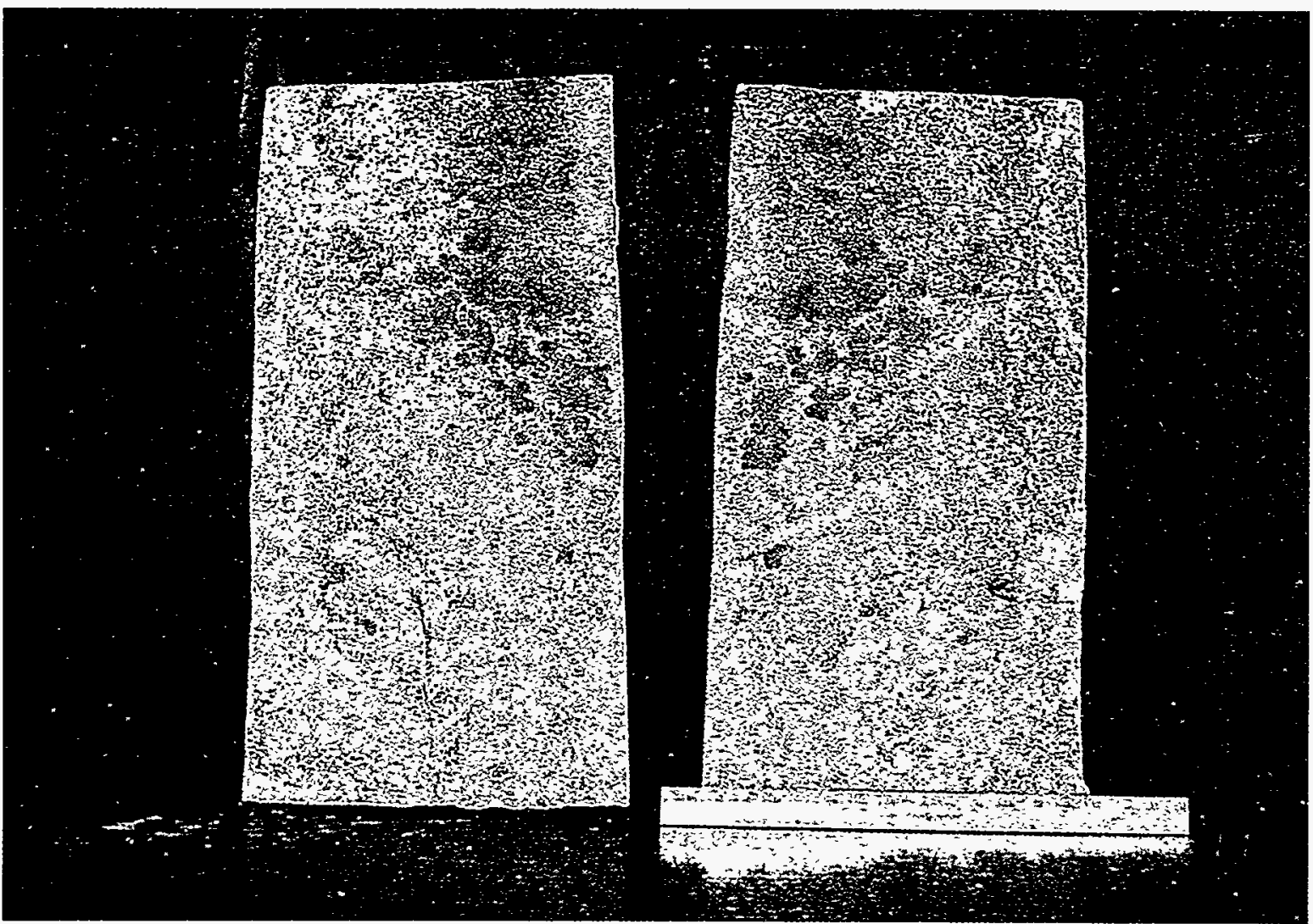

Figure 2.21. Wetting front instability during redistribution in a natural fracture (after Nicholl et al., 1994). 

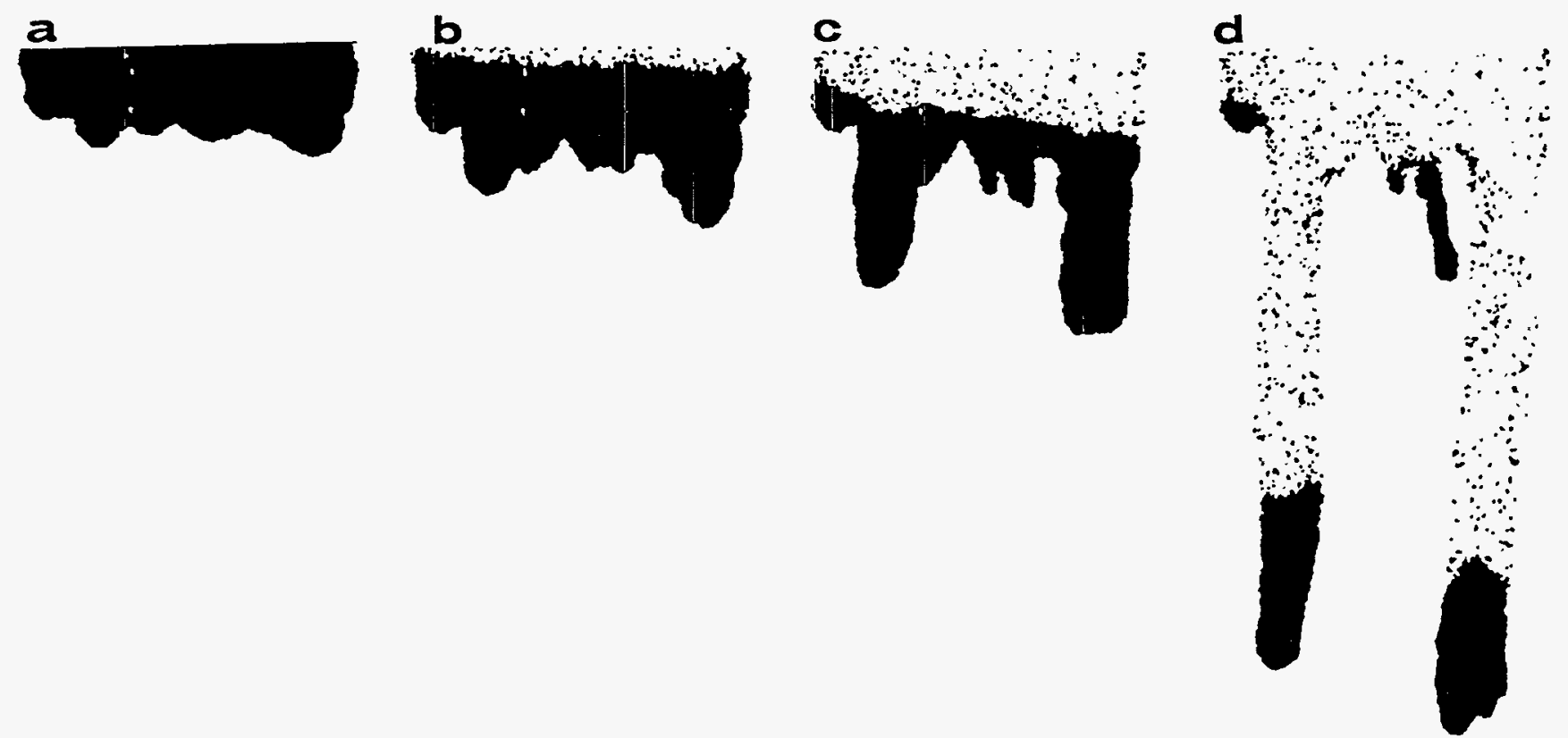

Figure 2.22. Finger development during redistribution in an initially dry artificial fracture at $\delta=139^{\circ}, L_{a}=9.43 \mathrm{~cm}$ : (a) $t=4 \mathrm{~s}$, (b) $t=7 \mathrm{~s}$, (c) $t=13 \mathrm{~s}$, (d) $t=39 \mathrm{~s}$ (after Nicholl et al., 1994).
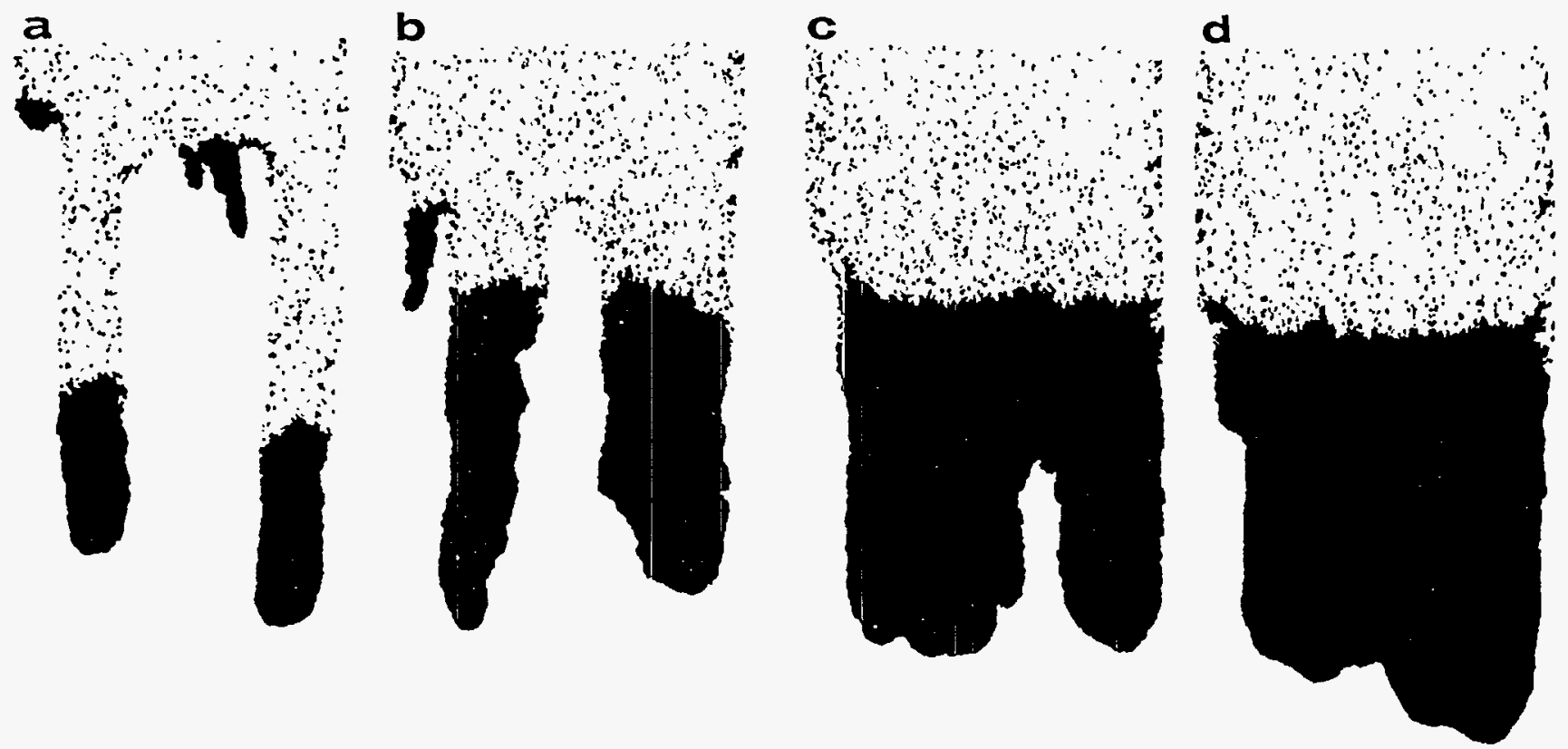

Figure 2.23. Effect of ponding depth $\left(L_{a}\right)$ on finger development in an artificial fracture during redistribution at $\delta=139^{\circ}$ : (a) $L_{a}=9.43 \mathrm{~cm}, t=31 \mathrm{~s}$, (b) $L_{a}=18.68$ $\mathrm{cm}, t=23 \mathrm{~s}$, (c) $L_{a}=28.17 \mathrm{~cm}, t=23 \mathrm{~s}$, (d) $L_{a}=33.47 \mathrm{~cm}, t=23 \mathrm{~s}$ (after Nicholl et al., 1994). 

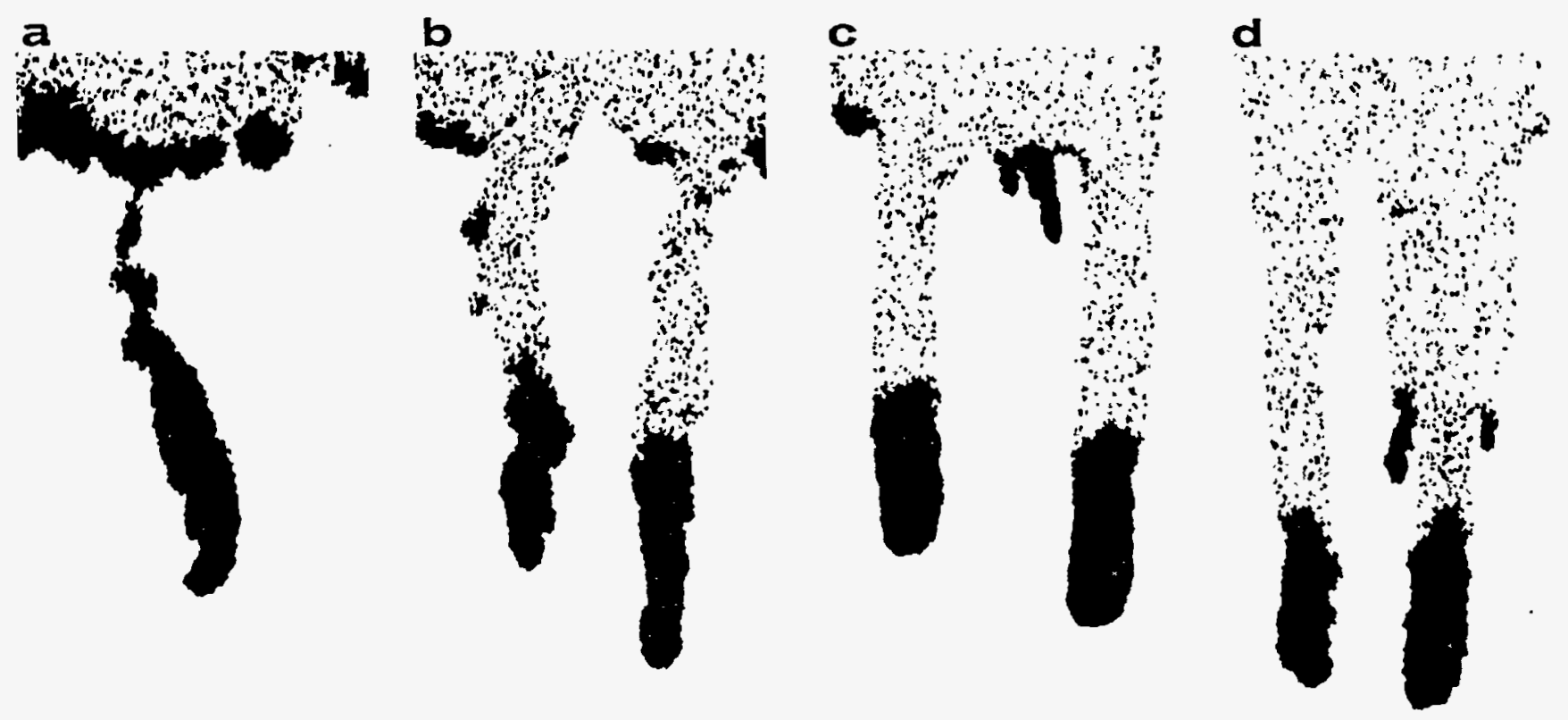

Figure 2.24. Effect of $\delta$ on finger development in an artificial fracture during redistribution: (a) $\delta=105^{\circ}, L_{a}=9.05 \mathrm{~cm}, t=1518 \mathrm{~s}$, (b) $\delta=120^{\circ}, L_{a}=9.85 \mathrm{~cm}$, $t=1045 \mathrm{~s}$, (c) $\delta=139^{\circ}, L_{a}=9.43 \mathrm{~cm}, t=31 \mathrm{~s}$, (d) $\delta=180^{\circ}, L_{a}=8.99 \mathrm{~cm}, t=$ 23 s (after Nicholl et al., 1994). 
Starr et al. (1978) conducted two field experiments at Windsor, Connecticut. The soil profile at the experimental site consists of sandy loam with a relatively fine layer over a coarser sublayer. The textural discontinuity between these two layers is at depths ranging from $60 \mathrm{~cm}$ to $90 \mathrm{~cm}$. The saturated hydraulic conductivity of the sublayer is about 20 times larger than that of the top layer. The water table was at a depth of $1.8 \mathrm{~m}$. In the first experiment, a steel cylinder $1.8 \mathrm{~m}$ in diameter was driven into the soil to a depth of $3.6 \mathrm{~m}$. Then $45 \mathrm{~cm}$ of water containing a green vegetable dye was applied abruptly at the soil surface. After it had infiltrated, successive layers of soil were removed from the cylinder and the dye pattern of each newly exposed surface was photographed. No distinct fingers were observed in the top soil. At a depth of $100 \mathrm{~cm}$, twelve greencolored fingers were revealed within the bottom soil, as shown in Figure 2.25. The fingers ranged from $5 \mathrm{~cm}$ to $20 \mathrm{~cm}$ in diameter and occupied only about $5 \%$ of the total crosssectional area. Upon further soil removal, each finger was noted to extend down to the water table. The capillary fringe above the water table appeared dark green beneath areas where fingers were most numerous and clear where fingers were absent.

In an adjacent second experiment, two neutron access tubes were installed in each of four $4.6 \times 6.1 \mathrm{~m}^{2}$ plots. The plots were additionally instrumented with two suction probes and one tensiometer at each of the seven depths $20,40,60,120,180,240$, and 300 $\mathrm{cm}$. The plots were covered with $5 \mathrm{~cm}$ of water until water contents and pressures stabilized throughout each profile. The remaining ponded water was then allowed to infiltrate and a $5 \mathrm{~cm}$ layer of $0.3 \mathrm{~N} \mathrm{CaCl}_{2}$ solution was applied at the soil surface. After this solution had infiltrated, the plots were again flooded to a depth of $5 \mathrm{~cm}$ with unlabeled water and kept at this level. Water was then sampled every $2-4$ hours and analyzed for chloride by titration with silver nitrate. The results suggested that chloride within the 20 $\mathrm{cm}$ to $60 \mathrm{~cm}$ depth range moved on the average at about half the mean pore-water velocity (flux divided by volumetric water content) in the shallower surface layer. The authors attributed this to air entrapment under the wetting front. In contrast to this relatively low average rate of chloride movement, several pulses have been recorded at depths of 120 and $180 \mathrm{~cm}$ soon after they had reached the $60 \mathrm{~cm}$ depth. This rapid movement of isolated solute pulses through the coarse layer is reminiscent of the first experiment where fingers formed below the $60 \mathrm{~cm}$ depth. The fact that nearly all probes recorded these pulses while, in the first experiment, fingers had occupied only $5 \%$ of the cross-sectional area may be due to the strong suction applied to the probes.

Glass et al. (1988) conducted field experiments in Haven loam at Long Island, 
Table 2.2. Summary of key field experiments.

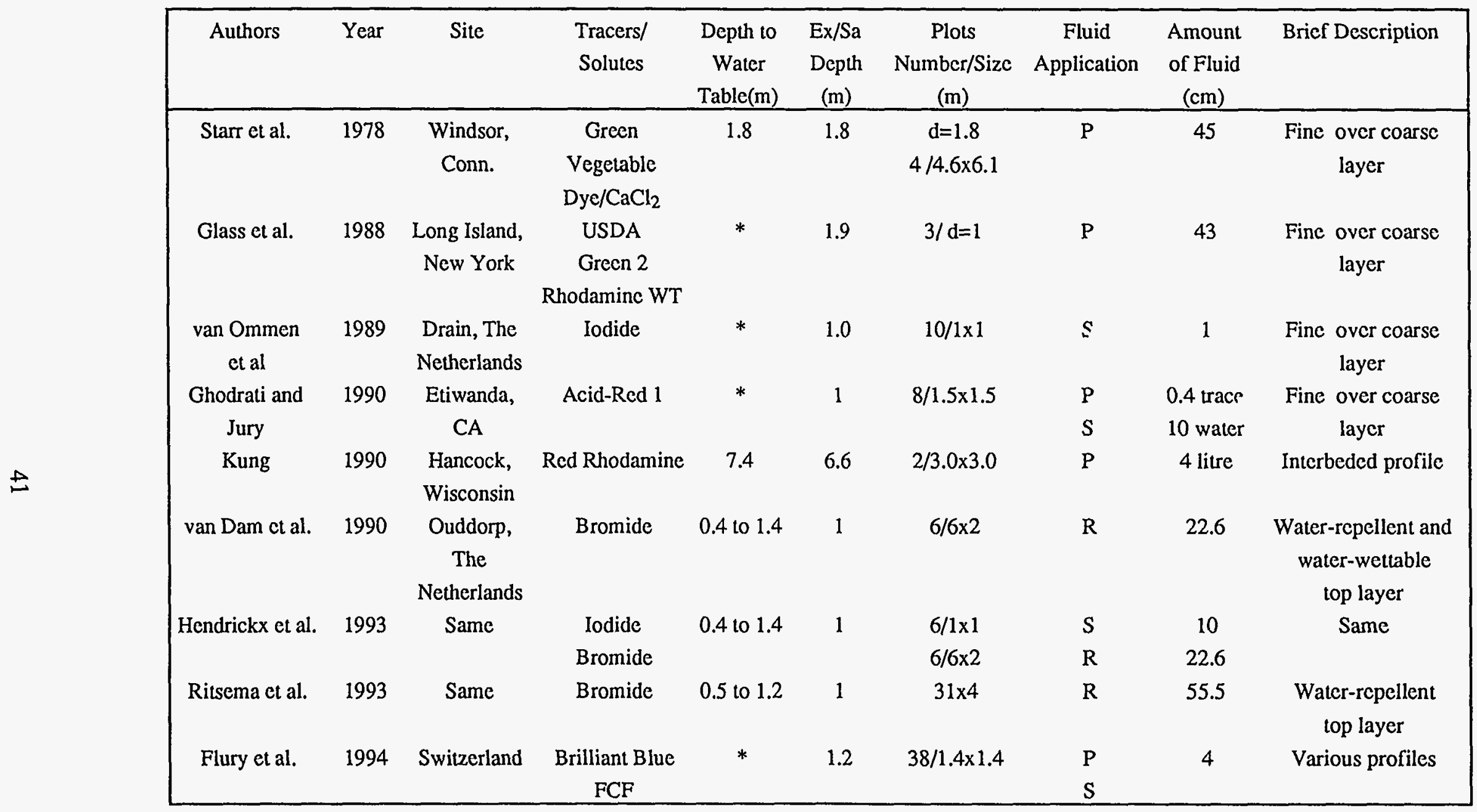

d: diameter, P: ponding, S: sprinkling, R: rainfall, *: unknown

Ex: excavation, Sa: sampling 


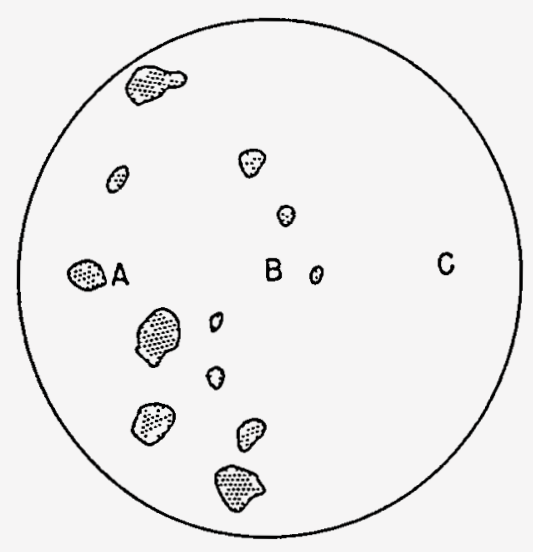

Figure 2.25. Isolated zones of dyed water at $100 \mathrm{~cm}$ depth after infiltration into a soil column at Windsor, Connecticut. The column is $1.8 \mathrm{~m}$ in diameter (after Starr et al., 1978). 
New York. At the site, a layer $30 \mathrm{~cm}$ thick with saturated hydraulic conductivity of $3.17 \mathrm{x}$ $10^{-4} \mathrm{~cm} / \mathrm{s}$ is found above a layer of much higher hydraulic conductivity in the range of 5.3 $\times 10^{-3}-1.06 \times 10^{-2} \mathrm{~cm} / \mathrm{s}$. Horizontal variability of hydraulic conductivities is small in comparison to this vertical contrast. An infiltration ring $1 \mathrm{~m}$ in diameter was forced into the top soil to a depth of $30 \mathrm{~cm}$. Infiltration was then initiated by applying $5 \mathrm{~cm}$ of water with $1 \%$ Rhodamine WT at the surface. A solution of $0.025 \%$ USDA Green 2 was added through a constant head device so as to maintain the ponding depth at $5 \mathrm{~cm}$ until a total of $43 \mathrm{~cm}$ had infiltrated. Whereas USDA Green 2 was found to move almost at the same rate as the wetting front in a laboratory test, Rhodamine WT is known to be strongly sorbing. Immediately after the cessation of ponding, the soil was carefully excavated to reveal residual flow patterns at various depths as shown in Figure 2.26. Finger-like structures are clearly evident, and the fraction of wetted area is seen to decrease with depth. Dark regions corresponding to the highly sorbing red dye appear to delineate core areas where most of the flow was taking place. Lightly shaded regions corresponding to the weakly sorbing green dye appear to delineate fringe areas where less flow took place. Water was found to penetrate deeper than either dye due to the ability of both dyes to sorb.

Van Ommen et al. (1989) conducted experiments at Drain in the Netherlands where a fine soil overlies a coarse one. Ten successive bursts of $1 \mathrm{~cm}$ iodide solution were applied to five plots $1 \mathrm{~m}^{2}$ in area by means of a high pressure sprinkling apparatus. Ponding was not observed during any application. Following this phase, the plots were covered to prevent any additional moisture flux at the surface. Two days later, the plots were excavated layer by layer. At each depth, the soil was covered with a thin layer of dry starch powder. After the powder became wet by soaking up soil water, it was sprayed with a chlorine containing solution. Wherever the soil water contained iodide, the latter oxidized to reveal a blue color. The percentage of blue area varied with depth in each plot as shown in Figure 2.27. Whereas the flow appeared to be uniform in the top layer, fingering was evident in the coarser underlying layer, as is also implied by the figure.

Ghodrati and Jury (1990) investigated the effect of irrigation method on fingering at a field site in Etiwanda, California. The soil at the site is layered with only slight textural variations horizontally. The first and third layers consist of loamy sand with saturated hydraulic conductivities between $1.4 \times 10^{-3} \mathrm{~cm} / \mathrm{s}$ and $2.5 \times 10^{-3} \mathrm{~cm} / \mathrm{s}$. The second layer, at a depth of about $100 \mathrm{~cm}-120 \mathrm{~cm}$ consists of gravel and coarse sand with a saturated hydraulic conductivity of $1.6 \times 10^{-2} \mathrm{~cm} / \mathrm{s}$. Based on visual observations, the profile was found to be devoid of any cracks, fissures, distinct root channels or earthworm 

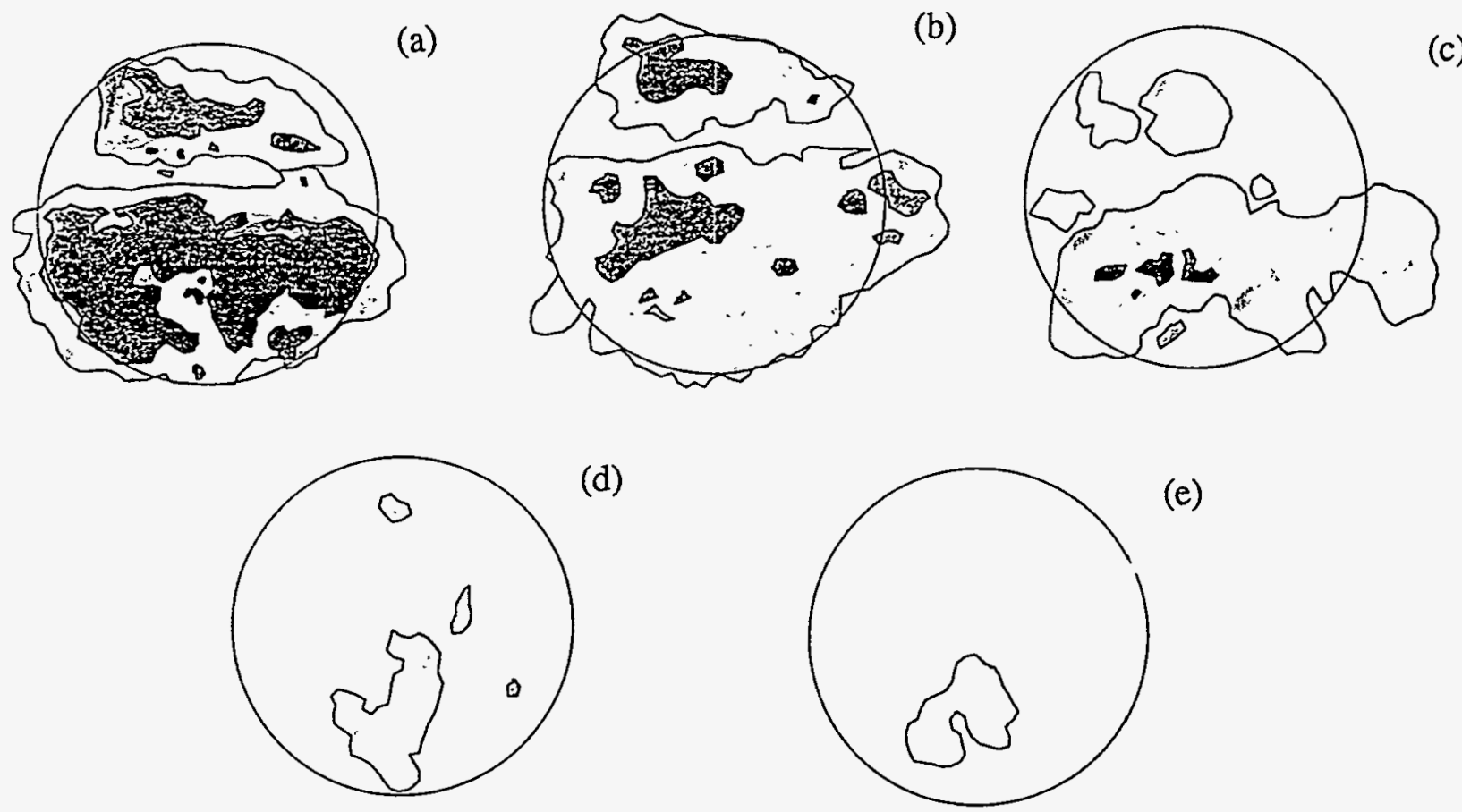

(e)

Figure 2.26. Horizontal dye patterns at depths of (a) $50 \mathrm{~cm}$, (b) $70 \mathrm{~cm}$, (c) $90 \mathrm{~cm}$, (d) $115 \mathrm{~cm}$, and (e) $140 \mathrm{~cm}$. Circle represents shallow infiltration ring, gray areas regions dyed green, dark areas regions dyed red (after Glass et al., 1988).

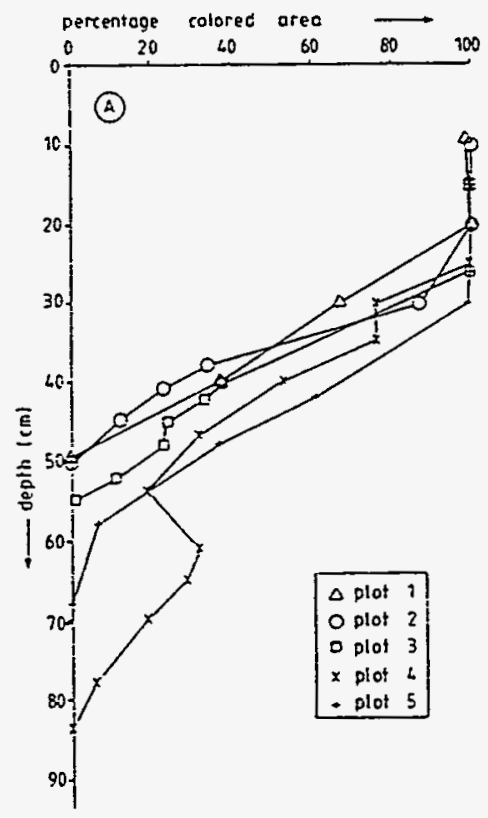

Figure 2.27. Percent blue area versus soil depth in five plots (after van Ommen et al., 1989) 
burrows. Eight soil plots of area $1.5 \times 1.5 \mathrm{~m}^{2}$ were prepared in two groups of four, one group receiving sprinkler irrigation and the other flood irrigation. Using a trencher, the upper part of two plots in each group were thoroughly disturbed to a depth of $30 \mathrm{~cm}-40$ $\mathrm{cm}$. After trenching, the disturbed plots were leveled and compacted slightly. A $0.4 \mathrm{~cm}$ pulse of Acid-Red 1 solution at a concentration of $80 \mathrm{gr} / \mathrm{kg}$ was applied uniformly to the surfaces of each plot. The plots were then irrigated with $10 \mathrm{~cm}$ of water, one group by sprinkling $2 \mathrm{~cm}$ of water each day for 5 days at a rate of $1.4 \times 10^{-4} \mathrm{~cm} / \mathrm{s}$, the other by flooding. Six days after application, all plots were excavated to a depth of $1 \mathrm{~m}$. The observed horizontal and vertical distribution of dye clearly indicated that fingering took place in each case. Finger widths ranged from $5 \mathrm{~cm}$ to $20 \mathrm{~cm}$. Fingering was less pronounced under sprinkling than under flooding in undisturbed plots. It was most pronounced where flood irrigation occurred over disturbed surfaces.

Kung (1990) conducted field experiment down to a depth of $6.6 \mathrm{~m}$ at the Hancock Agriculture Research Station in Wisconsin. Two $3.0 \times 3.6 \mathrm{~m}^{2}$ plots, each consisting of four potato rows, were studied. The soil profiles down to $1.1 \mathrm{~m}$ showed no distinct layering. Below this depth down to water table at $7.4 \mathrm{~m}$, there were discrete deposits of glacial-outwash under both plots with horizontal dimensions ranging from $1 \mathrm{~m}$ to $3 \mathrm{~m}$. Coarser deposits were often embedded as lenses among several interbeded fine and/or medium grained deposits. Infiltration was initiated by pouring 2.7 liters of water containing 3\% Rhodamine WT into furrows between the rows of potato. The application was repeated four times at ten-day intervals. Eighty days after the first dye application and one week after potato harvesting, the plots were excavated layer by layer. The red dye solution was found to have moved in vertical plumes down to $1.0 \mathrm{~m}$ with very little lateral spreading. Between $1.1 \mathrm{~m}$ and $1.4 \mathrm{~m}$, some of the red plumes spread out and merged with each other. Water and solute occupied less than $50 \%$ of the soil volume between $1.5 \mathrm{~m}$ and $2.0 \mathrm{~m}$, less than $10 \%$ between 3.0 and $3.5 \mathrm{~m}$, and less than $1 \%$ between $5.6 \mathrm{~m}$ and 6.6 $\mathrm{m}$. Both hydrodynamic instability and soil heterogeneity appeared to have played a role in controlling the flow.

Field studies on fingering in water-repellent soils have been carried out extensively at Ouddorp in The Netherlands (e.g., van Dam et al., 1990; Hendrickx et al., 1993; Ritsema et al., 1993; and Ritsema and Dekker, 1994). The local sandy soils are of marine origin and often contain water-repellent top layers. Studies were conducted on two adjacent parcels covered with grass, one with and one without a water-repellent top layer. The degree of water repellency was characterized by placing a drop of water at the surface 
of a soil sample and measuring the time required for it to move into the sample. Water repellency was found to decrease with depth and to disappear below $30 \mathrm{~cm}$. Depth to water table at the site fluctuated between $40 \mathrm{~cm}$ and $140 \mathrm{~cm}$ during the experiments.

Van Dam et al. (1990) and Hendrickx et al. (1993) applied 15 liters of a $\mathrm{KBr}$ solution with a concentration of $11 \mathrm{gr} / \mathrm{l}$ to each of three $6 \times 2 \mathrm{~m}^{2}$ plots in each parcel at the above site by sprinkling without any ponding. Just before sprinkling and after $8,13,35$ and 57 days, water and bromide contents were sampled at various locations and depths by mechanically driving hollow cylindrical samplers of length $100 \mathrm{~cm}$ and diameter $10 \mathrm{~cm}$ into the soil. Bromide was found to have reached the water table earlier below the waterrepellent soil than below the wettable soil. Following $62 \mathrm{~mm}$ of rain two weeks after application, the bromide has moved down to a depth of $60 \mathrm{~cm}$ below the wettable soil. Below the water-repellent soil, a mean concentration of $20 \mathrm{mg} / \mathrm{l}$ was measured in the groundwater at a depth of $80 \mathrm{~cm}$. At the same time, some of the bromide remained close to the surface of the water-repellent soil. After five weeks and a total precipitation of 120 $\mathrm{mm}$, there was six to thirteen times more bromide in the groundwater under waterrepellent soils than under wettable soils. In a similar study at the same site, Ritsema et al. (1993) compared the ratios of total bromide in each vertical soil core under the waterrepellent soil to that sprayed on the core surface. On day 11 , the ratio in twenty cores ranged from 0.4 to 1.7. Ten of these cores contained more bromide than applied to them, indicating that bromide applied uniformly at the soil surface has spread in a nonuniform fashion through the unsaturated zone. Ritsema and Dekker (1994) showed that fingers develop preferentially where water repellency in the shallow top layer is reduced.

Hendrickx et al. (1993) applied $10 \mathrm{~cm}$ of an iodide solution to each of three $1 \mathrm{~m}^{2}$ plots on each parcel for two days at a low enough rate to avoid ponding. The plots were then excavated layer by layer to a depth of $90 \mathrm{~cm}$ and the cleared surfaces treated by the same iodide coloring technique described earlier. Whereas the wettable top layers were completely blue down to $30 \mathrm{~cm}$, in the water-repellent top layers the blue areas covered only $35 \%$ of the total area at a depth of $5 \mathrm{~cm}$ and about $65 \%$ at a depth of $30 \mathrm{~cm}$. Measured volumetric water contents in the water-repellent layer were significantly higher within than outside the blue areas. Below $30 \mathrm{~cm}$, the percentage of blue area decreased with depth in both the wettable and the water-repellent parcels.

Flury et al. (1994) conducted experiments in agricultural soils at fourteen field sites throughout Switzerland. The soils ranged from structureless to well structured and from fine granular to very coarse angular and blocky. Each site consisted of two $1.4 \times 1.4$ 
$\mathrm{m}^{2}$ plots, one covered with a plastic roof for two months prior to the experiment so as to obtain different initial water contents in each. Forty millimeters of water containing Brilliant Blue FCF were applied within 8 hours onto the plots with a sprinkling apparatus. At five of the fourteen field sites, two additional circular plots with a diameter of $1 \mathrm{~m}$ were flooded to a depth of $4 \mathrm{~cm}$. One day after irrigation, all plots were excavated and the soil examined along vertical $1 \times 1 \mathrm{~m}^{2}$ profiles. In most soils, dyed water moved along preferential paths. In some soils, the dye remained within the top $50 \mathrm{~cm}$, in others it moved below $1 \mathrm{~m}$. Structured soils were observed to be more prone to fingering and deep penetration than nonstructured soils. There was no clear effect of initial soil water content on flow pattern. In soils that contained cracks and earthworm channels, ponding resulted in less regular flow patterns and deeper penetration depths than sprinkling. In structureless soils, differences between flow patterns generated by ponding and sprinkling were less pronounced.

The above field experiments demonstrate that fingering during infiltration is the rule rather than the exception. The phenomenon occurs under sprinkling as well ponding in seemingly uniform as well as heterogeneous soils which may or may not contain discernible cracks or channels, and may be either wettable or water repellent. It appears that, in the field, fingering results from the combined actions of hydrodynamic instability and medium heterogeneity. 


\section{THEORETICAL ANALYSES}

We start this chapter with a brief description of the early stability analysis by Hill (1952). This will serve as a relatively simple introduction to the more general analyses presented later in this chapter.

Consider a fluid of viscosity $\mu_{2}$ and density $\rho_{2}$ displacing another fluid of viscosity $\mu_{1}$ and density $\rho_{1}$ upward. The immiscible fluids are separated by a sharp horizontal interface moving vertically along the $z$ axis with velocity $U$ (Figure 3.1). Let $\phi_{j}$ be the velocity potential in the $j$-th fluid so that fluid velocity is given by $v_{j}=-d \phi_{j} / d z$. Mass continuity requires that $\phi_{j}$ satisfy the Laplace equation

$$
\frac{d^{2} \phi_{j}}{d z^{2}}=0
$$

Hence $\phi_{j}$ is linear in $z$,

$$
\phi_{j}=-U z ; j=1,2 .
$$

Darcy's law implies that $\phi_{j}$ is given by

$$
\phi_{j}=\frac{k_{j}}{\mu_{j}}\left(p_{j}+\rho_{j} g z\right)
$$

where $k_{j}$ is permeability, $p_{j}$ is pressure, and $g$ is acceleration due to gravity. Substituting (3.2) into (3.3) yields the following pressure difference across the interface,

$$
\Delta p^{0}=p_{2}-p_{1}=\left(\frac{\mu_{1}}{k_{1}}-\frac{\mu_{2}}{k_{2}}\right) U z+\left(\rho_{1}-\rho_{2}\right) g z
$$

Suppose that a small discrete perturbation $\delta z$ develops along the interface. The corresponding pressure difference is

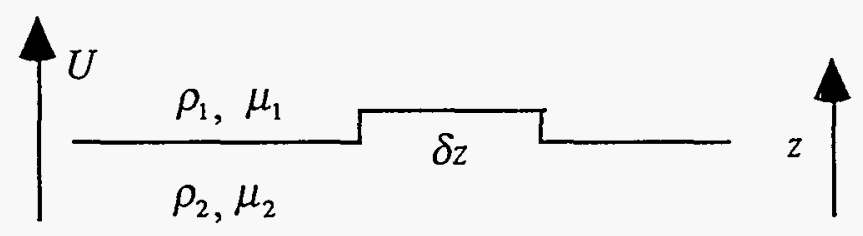

Figure 3.1. Sketch of disturbed interface. 


$$
\Delta p=\left(\frac{\mu_{1}}{k_{1}}-\frac{\mu_{2}}{k_{2}}\right) U(z+\delta z)+\left(\rho_{1}-\rho_{2}\right) g(z+\delta z) .
$$

The perturbation dies out if $\Delta p<\Delta p^{0}$ but grows if $\Delta p>\Delta p^{0}$. In other words, the interface becomes unstable if

$$
\Delta p-\Delta p^{0}=\left[\left(\frac{\mu_{1}}{k_{1}}-\frac{\mu_{2}}{k_{2}}\right) U+\left(\rho_{1}-\rho_{2}\right) g\right] \delta z>0
$$

or

$$
\left(\frac{\mu_{1}}{k_{1}}-\frac{\mu_{2}}{k_{2}}\right) U+\left(\rho_{1}-\rho_{2}\right) g>0 \text {. }
$$

We see that if $k_{1}=k_{2}$ and $U$ is positive (directed upward), viscosity has a destabilizing effect when a less viscous fluid displaces a more viscous fluid and a stabilizing effect otherwise. The same is true when $U$ is negative. Gravity has a destabilizing effect when the denser fluid is at top, and a stabilizing effect in the opposite case, regardless of $U$. The critical velocity $U_{c}$, above which the interface becomes unstable, is

$$
U_{c}=-\frac{\left(\rho_{1}-\rho_{2}\right) g}{\mu_{1} / k_{1}-\mu_{2} / k_{2}} \text {. }
$$

This explains the experimental observations in Figure 2.2.

In the special case where liquid displaces air downward, $U_{c} \approx-K_{2}$ where $K_{2}$ is the hydraulic conductivity of the liquid. Hence the interface is stable when the magnitude of the velocity is larger than $K_{2}$, unstable when it is smaller.

\subsection{Instability in an Ideal Fracture}

The analysis of Hill (1952) does not account for surface tension. A rigorous hydrodynamic analysis which accounts for surface tension across a horizontal interface in a vertical Hele-Shaw cell was performed by Saffman and Taylor (1958). Neuman (1982) extended their analysis to cells and interfaces having arbitrary orientations. His analysis, which also applies to ideal fractures, proceeds as follows.

Consider a fracture of infinite extent and constant aperture $b$. Two immiscible fluids, 1 and 2, completely fill the gap between the fracture walls. The fluids are separated 
by a sharp interface coinciding with the $x^{*}$ axis (Figure 3.2) where $x^{*}$ and $y^{*}$ form a Cartesian system of coordinates in the fracture plane. The fluids move at a uniform velocity $U$ parallel to the $x$ axis which forms an angle $\varphi$ with $x^{*} ; x$ and $y$ form another Cartesian system in the fracture plane whose origin coincides with that of $x^{*}$ and $y^{*}$. The relationship between the two coordinate systems is $x^{*}$

$$
\begin{aligned}
& x=x * \cos \varphi+y * \sin \varphi \\
& y=-x * \sin \varphi+y * \cos \varphi
\end{aligned}
$$

The angle between $x$ and $y$ and the vertical (a vector pointing in a direction opposite to that of gravity) are $\alpha$ and $\beta$, respectively.

Assume that at time $t=0$ the interface is perturbed slightly into a wave form (Figure 3.3). A single harmonic of this wave can be described by $y^{*}=\eta\left(x^{*}, t\right)$ where

$$
\eta\left(x^{*}, t\right)=\varepsilon \mathrm{e}^{i n x^{*}+\sigma t} .
$$

Here $i=\sqrt{-1}, n$ is wave number or period at a given $t$ (equal to $2 \pi / \lambda$ where $\lambda$ is wavelength), $\varepsilon$ is a small positive constant, and $\sigma$ is amplification or growth factor. The wave grows in magnitude if $\sigma$ is positive and diminishes if $\sigma$ is negative.

Suppose that within each fluid, $j=1$ and 2 , there exists a velocity potential, $\phi_{j}$. The velocity potential satisfies the Laplace equation

$$
\nabla^{2} \phi_{j}=0 ; j=1,2 \text {. }
$$

The kinematic condition at the interface, $\mathrm{D}\left(y^{*}-\eta\right) / \mathrm{D} t=0$ at $y^{*}=\eta$ where $\mathrm{D}$ denotes hydrodynamic differential, can be expressed to a first order of approximation as

$$
-\frac{\partial \phi_{1}}{\partial y^{*}}=-\frac{\partial \phi_{2}}{\partial y^{*}}=U \sin \varphi+\sigma \varepsilon \mathrm{e}^{i n x^{*}+\sigma t}
$$

The solutions of (3.11) and (3.12) which satisfy the additional requirement that the disturbance vanishes far from the interface at $y^{*}= \pm \infty$ are

$$
\begin{aligned}
& \phi_{1}=-U\left(x^{*} \cos \varphi+y^{*} \sin \varphi\right)+\frac{\sigma \varepsilon}{n} \mathrm{e}^{i n x^{*}-n y^{*}+\sigma t} \\
& \phi_{2}=-U\left(x^{*} \cos \varphi+y^{*} \sin \varphi\right)-\frac{\sigma \varepsilon}{n} \mathrm{e}^{i n x^{*}+n y^{*}+\sigma t} .
\end{aligned}
$$




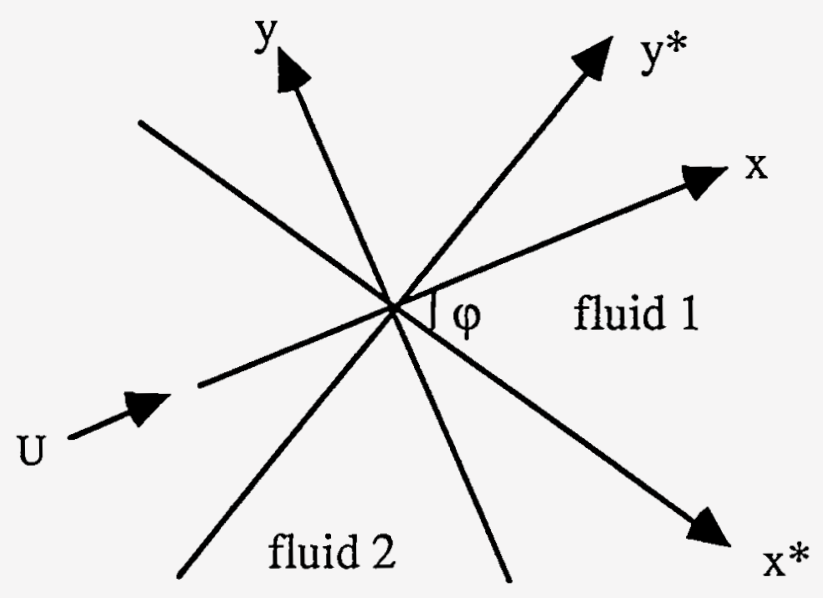

Figure 3.2. Interface between fluids 1 and 2 .

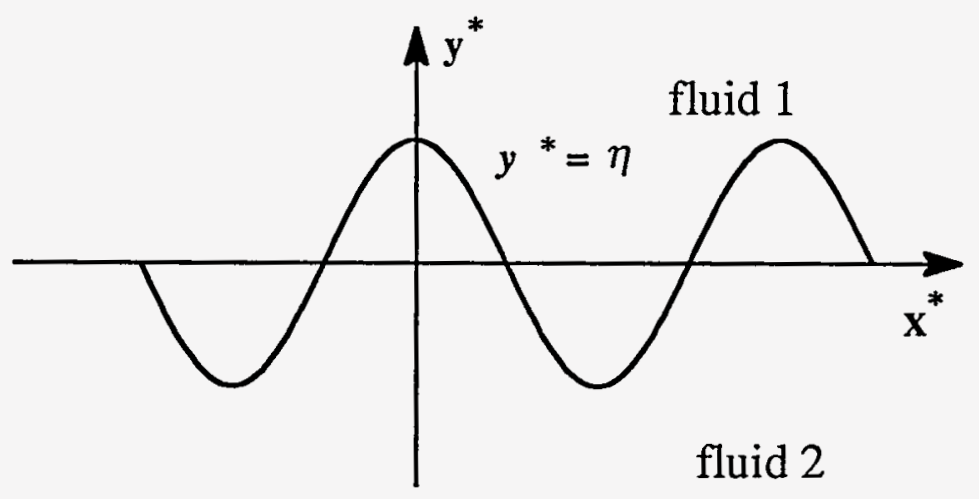

Figure 3.3. Wave like disturbance. 
At the interface where $y^{*}=\eta,(3.13)$ can be approximated to first order by

$$
\begin{aligned}
& \phi_{1}=-U(x * \cos \varphi+\eta \sin \varphi)+\frac{\sigma}{n} \eta \\
& \phi_{2}=-U(x * \cos \varphi+\eta \sin \varphi)-\frac{\sigma}{n} \eta .
\end{aligned}
$$

The dynamic condition at the interface, in the presence of surface tension, is usually given by Laplace's formula. For sufficiently small wave amplitudes, this formula is written as (c.f., McCormack and Crane, 1973; Saffman and Taylor, 1958)

$$
p_{1}-p_{2}=T \frac{\partial^{2} \eta}{\partial x^{2}} \pm \frac{2 T \cos \theta^{\prime}}{b}
$$

where $T$ is surface tension coefficient, $\theta^{\prime}$ is contact angle between fracture wall and wetting fluid, and the sign is positive or negative depending on whether 2 or 1 is the wetting fluid. Whereas the first term on the right represents surface tension due to the lateral curvature of the interface, the second term represents surface tension due to transverse curvature. We assume that the contact angle, $\theta^{\prime}$, is constant. Park and Homsy (1984) claim that (3.17) is valid only at equilibrium in the absence of flow. For flow at small capillary numbers they suggest replacing $T$ by $(\pi / 4) T$ in (3.17). As this contradicts (3.17) in the limit as velocity approaches zero, we retain the original form.

When Darcy's law holds, the velocity potential takes the form

$$
\phi_{j}=\frac{k_{j}}{\mu_{j}}\left\{p_{j}+\rho_{j} g[(x * \cos \varphi+y * \sin \varphi) \cos \alpha+(-x * \sin \varphi+y * \cos \varphi) \cos \beta]+f_{j}(t)\right\}
$$

where $f_{j}(t)$ is an arbitrary function of time and the term in square brackets represents elevation above the origin of the coordinates. Substituting (3.15) and (3.16) into (3.18), solving for $p_{j}$, substituting the result into (3.17), and rearranging yields

$$
A \eta-\frac{\partial^{2} \eta}{\partial x^{2}}=B+C x^{*}
$$

where

$$
A=\frac{1}{T}\left[\left(\frac{\mu_{1}}{k_{1}}+\frac{\mu_{2}}{k_{2}}\right) \frac{\sigma}{n}-\left(\frac{\mu_{1}}{k_{1}}-\frac{\mu_{2}}{k_{2}}\right) U \sin \varphi-\left(\rho_{1}-\rho_{2}\right) g(\sin \varphi \cos \alpha+\cos \varphi \cos \beta)\right]
$$




$$
\begin{aligned}
& B= \pm \frac{2 \cos \theta^{\prime}}{b}-\frac{f_{2}(t)-f_{1}(t)}{T} \\
& C=\frac{1}{T}\left[\left(\frac{\mu_{1}}{k_{1}}-\frac{\mu_{2}}{k_{2}}\right) U \cos \varphi+\left(\rho_{1}-\rho_{2}\right) g(\cos \varphi \cos \alpha-\sin \varphi \cos \beta)\right]
\end{aligned}
$$

The solution of (3.19) is

$$
\eta=c_{1} \mathrm{e}^{\sqrt{A} x^{*}}+c_{2} \mathrm{e}^{-\sqrt{A} x^{*}}+\frac{B+C x^{*}}{A} .
$$

For this to have the same form as (3.10), we must set $A=-n^{2}, B=0$ and $C=0$. These give the two desired stability criteria,

$$
\begin{gathered}
\left(\frac{\mu_{1}}{k_{1}}+\frac{\mu_{2}}{k_{2}}\right) \frac{\sigma}{n}=\left(\frac{\mu_{1}}{k_{1}}-\frac{\mu_{2}}{k_{2}}\right) U \sin \varphi+\left(\rho_{1}-\rho_{2}\right) g(\sin \varphi \cos \alpha+\cos \varphi \cos \beta)-T n^{2} \\
\left(\frac{\mu_{1}}{k_{1}}-\frac{\mu_{2}}{k_{2}}\right) U \cos \varphi+\left(\rho_{1}-\rho_{2}\right) g(\cos \varphi \cos \alpha-\sin \varphi \cos \beta)=0 \\
f_{2}(t)-f_{1}(t)= \pm \frac{2 T \cos \theta^{\prime}}{b} .
\end{gathered}
$$

The first of these criteria, (3.21), shows that for $\sigma$ to be positive so as to cause the wave to grow, one must have

$$
\left(\frac{\mu_{1}}{k_{1}}-\frac{\mu_{2}}{k_{2}}\right) U \sin \varphi+\left(\rho_{1}-\rho_{2}\right) g(\sin \varphi \cos \alpha+\cos \varphi \cos \beta)>0 .
$$

A sufficient condition for the wave to grow is that the wave number not exceed a certain critical value,

$$
n^{2}<n_{c}^{2}
$$

where

$$
n_{c}^{2}=\frac{1}{T}\left[\left(\frac{\mu_{1}}{k_{1}}-\frac{\mu_{2}}{k_{2}}\right) U \sin \varphi+\left(\rho_{1}-\rho_{2}\right) g(\sin \varphi \cos \alpha+\cos \varphi \cos \beta)\right] .
$$

Equations (3.24) and (3.25) show that when $n_{c}^{2}<0$, all the harmonics will decay, and the interface will remain smooth. When $n_{c}{ }^{2}>0$, only harmonics having a sufficiently large wave number (or a sufficiently short wavelength) will decay, whereas the others will 
grow. Surface tension is seen to have a stabilizing effect on the interface because the larger is $T$, the smaller is $n_{c}{ }^{2}$, and thus the wider is the range of stable harmonics.

By taking the derivative of $\sigma$ with respect to $n$ in (3.21) and setting it equal to zero, one finds that the fastest growing harmonic is that with wave number

$$
n_{m}=\frac{n_{c}}{\sqrt{3}}
$$

In the absence of surface tension, there is no fastest growing wavelength. The shorter is the wavelength, the faster its amplitude grows. According to Chuoke et al. (1959), the fastest growing wave number will initially dominate and will be the first to show up in an experiment. The corresponding wavelength

$$
\lambda_{m}=\frac{2 \pi}{n_{m}}=\frac{2 \sqrt{3} \pi}{n_{c}}
$$

is often used to characterize finger size.

Regardless of whether the disturbance grows or decays, the orientation of the interface relative to that of the velocity must be such as to satisfy (3.22). If $\varphi$ at $t=0$ is such that this criterion is violated, the interface will rotate until a dynamic equilibrium is achieved and (3.22) is satisfied. This rotational aspect of stability is not evident in the analysis of Saffman and Taylor (1958) because the angle between the velocity vector and the interface is prescribed a priori. In the particular case where the fracture is vertical $(\alpha=0 ; \beta=\pi / 2)$ then $\cos \alpha=\sin \beta$. If also $\cos \varphi \neq 0$, and $\left(\rho_{1}-\rho_{2}\right) g \cos \beta \neq 0$, then (3.22) can be written as

$$
\tan \varphi=\tan \beta+\frac{U\left(\mu_{1} / k_{1}-\mu_{2} / k_{2}\right)}{\left(\rho_{1}-\rho_{2}\right) g \cos \beta}
$$

which is analogous to equation (20) of Jacquard and Séguier (1962) who derived it via a completely different method based on considerations of static equilibrium. If $U$ is horizontal, the interface is inclined, its slope being given by

$$
\tan \varphi=\frac{U\left(\mu_{1} / k_{1}-\mu_{2} / k_{2}\right)}{\left(\rho_{1}-\rho_{2}\right) g}
$$

Equation (3.22) shows that for the interface to be orthogonal to the velocity vector (i.e., for $\varphi$ to be $\pi / 2$ ), $\cos \beta$ must be equal to zero. This means that the $y$ axis must coincide 
with the strike of the fracture, and the $x$ axis with its dip. In other words, the interface is perpendicular to the velocity if and only if the latter is directed parallel to the fracture dip. In all other cases, the fingers will grow in a direction which is not coincident with that of the velocity vector, unless forced to do otherwise by lateral boundaries.

In the particular case where the fracture is vertical and the velocity is directed upward ( $\alpha=0 ; \beta=\pi / 2$ ), (3.22) implies that $\varphi$ is $\pi / 2$, indicating that the interface is horizontal. Equation (3.21) reduces to the criterion derived by Saffman and Taylor (1958),

$$
\left(\frac{\mu_{1}}{k_{1}}+\frac{\mu_{2}}{k_{2}}\right) \frac{\sigma}{n}=\left(\frac{\mu_{1}}{k_{1}}-\frac{\mu_{2}}{k_{2}}\right) U+\left(\rho_{1}-\rho_{2}\right) g-T n^{2} .
$$

In order for $\sigma$ to be positive so as to cause the wave to grow, one must have

$$
\left(\frac{\mu_{1}}{k_{1}}-\frac{\mu_{2}}{k_{2}}\right) U+\left(\rho_{1}-\rho_{2}\right) g>0 \text {. }
$$

This is the same as (3.7) derived earlier by Hill (1952) and, like it, explains the experimental observations in Figure 2.2. The most unstable wavelength is given by

$$
\lambda_{m}=2 \pi\left[\frac{3 T}{\left(\mu_{1} / k_{1}-\mu_{2} / k_{2}\right) U+\left(\rho_{1}-\rho_{2}\right) g}\right]^{1 / 2} .
$$

Clearly, finger wavelength increases with $T$, and increases or decreases with $U$ depending on the sign of $\left(\mu_{1} / k_{1}-\mu_{2} / k_{2}\right)$. The phenomenon has been observed in many experiments (e.g., Chuoke et al., 1959, Glass et al., 1989b). If we further set $k_{1}=k_{2}=k$, then (3.31) simplifies to

$$
\frac{\sigma}{U}=\left[\frac{\mu_{1}-\mu_{2}}{\mu_{1}+\mu_{2}}+\frac{\left(\rho_{1}-\rho_{2}\right) g k}{\left(\mu_{1}+\mu_{2}\right) U}\right] n-\frac{k T}{\left(\mu_{1}+\mu_{2}\right) U} n^{3} .
$$

Upon defining a characteristic length $L$ and a characteristic time $L / U$, the above can be written in dimensionless form as

$$
\sigma=(A+D R) n^{\prime}-\frac{(A+1)}{2 C a^{\prime}} n^{\prime 3}
$$


where $\sigma^{\prime}=\sigma L / U$ and $n^{\prime}=n L$. The dimensionless groups $A=\left(\mu_{1}-\mu_{2}\right) /\left(\mu_{1}+\mu_{2}\right)$, $D R=\left(\rho_{1}-\rho_{2}\right) g k /\left[\left(\mu_{1}+\mu_{2}\right) U\right]$ and $C a^{\prime}=\mu_{1} U /(k T)$ have been defined in the introduction.

In the particular case where water displaces air downward $(\alpha=\pi, \beta=\varphi=\pi / 2$, $\left.\mu_{1} \approx 0, \rho_{1} \approx 0\right),(3.21)$ takes the form

$$
\frac{\sigma}{U} \approx\left(-1+\frac{\rho_{2} g k}{\mu_{2} U}\right) n-\frac{k T}{\mu_{2} U} n^{3} .
$$

Therefore the modified capillary number in this case shall be defined as $\mu_{2} U /(k T)$ rather than $\mu_{1} U /(k T)$. The most unstable wavelength for this special case is

$$
\lambda_{m}=\sqrt{3} \lambda_{c} \approx 2 \pi\left(\frac{3 T}{\rho_{2} g-\mu_{2} U / k}\right)^{1 / 2} .
$$

Here, finger wavelength increases with $U$.

\subsection{Instability in a Porous Medium}

An analysis of instability under immiscible displacement in a porous medium was conducted by Chuoke et al. (1959). The authors allowed flow to be other than vertical but assumed that the interface remains perpendicular to the direction of flow. We present below a more general analysis which shows that the interface is not generally at right angle to the velocity.

Consider an infinite porous volume occupied by two immiscible fluids separated by a sharp planar macroscopic interface. The interface is perpendicular to the $y^{*}$ axis of a Cartesian system $\left(x^{*}, y^{*}, z^{*}\right)$. It coincides with the $x^{*}$ and $z^{*}$ axes where $z^{*}$ is horizontal. The axes $x^{*}$ and $y^{*}$ form angles $\alpha$ and $\beta$ with the vertical (a vector pointing in a direction opposite to that of gravity). The fluids move at a uniform velocity $U$ parallel to the $x$ axis of another Cartesian system $(x, y, z)$ whose origin coincides with that of $\left(x^{*}, y^{*}, z^{*}\right)$. The $x$ axis is directed from fluid 2 to fluid 1 . The coordinates $y$ and $z$ lie in the plane normal to the flow direction and $z$ is horizontal. If $\varphi_{1}, \varphi_{2}, \varphi_{3}$ are the angles between $x$ and $x^{*}, y^{*}$, $z^{*}$, then

$$
x=x * \cos \varphi_{1}+y * \cos \varphi_{2}+z * \cos \varphi_{3} .
$$




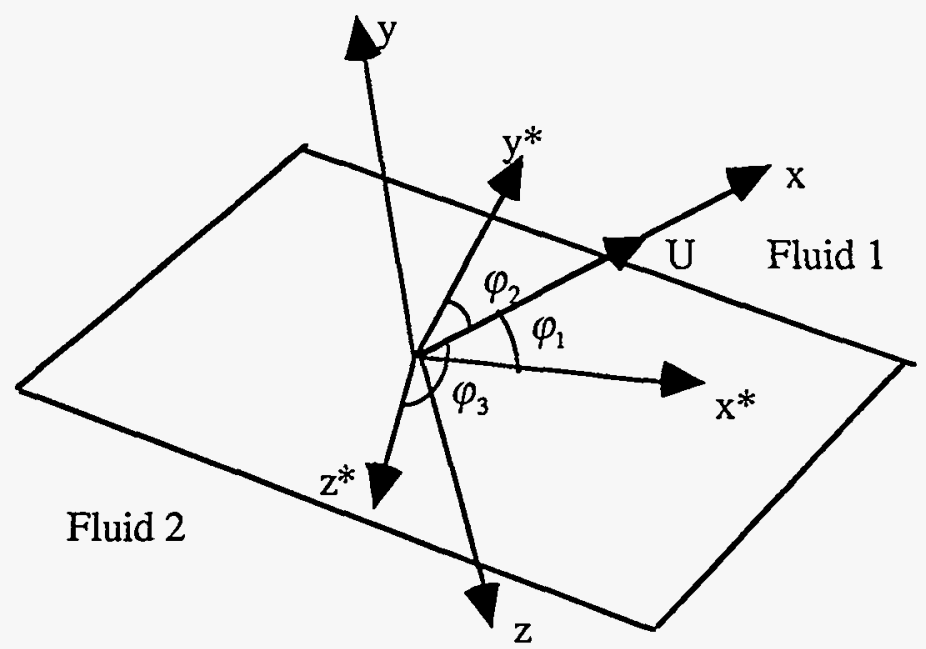

Figure 3.4. Interface between fluids 1 and 2 in 3-D domain

Assume that, at $t=0$, the interface is perturbed into a wave form. A single harmonic of this wave is described by

$$
y^{*}=\eta\left(x^{*}, z^{*}, t\right)=\varepsilon \mathrm{e}^{i\left(n x^{*}+m z^{*}\right)+\sigma t}
$$

where $n$ and $m$ are wave numbers corresponding to $x$ and $y, \varepsilon$ is a small amplitude, and $\sigma$ is a growth (or decay) factor. The kinematic condition at the interface, $\mathrm{D}\left(y^{*}-\eta\right) / \mathrm{D} t=0$ at $y^{*}=\eta$ where $\mathrm{D}$ denotes hydrodynamic differential, can be expressed to a first order of approximation as

$$
-\frac{1}{\Delta \theta_{1}} \frac{\partial \phi_{1}}{\partial y^{*}}=-\frac{1}{\Delta \theta_{2}} \frac{\partial \phi_{2}}{\partial y^{*}}=U \cos \varphi_{2}+\sigma \varepsilon \mathrm{e}^{i\left(n x^{*}+m z^{*}\right)+\sigma t},
$$

where $\Delta \theta_{i}$ is the increase in volumetric fluid content $\theta_{i}$ following displacement. Fluid 2 ahead of the interface, and fluid 1 behind the interface, are assumed to be immobile until the remaining pore space has been invaded by the same fluid, at which time they become mobile.

The solutions of (3.40) together with the Laplace equations $\nabla^{2} \phi_{j}=0$, subject to the requirement of zero disturbance far from the interface at $y^{*}= \pm \infty$, are 


$$
\begin{aligned}
& \phi_{1}=\left[-U x+\frac{\sigma \varepsilon}{M} \mathrm{e}^{i\left(n x^{*}+m z^{*}\right)-M y^{*}+\sigma t}\right] \Delta \theta_{1} \\
& \phi_{2}=\left[-U x-\frac{\sigma \varepsilon}{M} \mathrm{e}^{i\left(n x^{*}+m z^{*}\right)+M y^{*}+\sigma t}\right] \Delta \theta_{2}
\end{aligned}
$$

where $M^{2}=n^{2}+m^{2}$. At the interface, where $y^{*}=\eta$, (3.41) and (3.42) can be approximated to first order by

$$
\begin{aligned}
& \phi_{1}=\left(-U x+\frac{\sigma}{M} \eta\right) \Delta \theta_{1} \\
& \phi_{2}=\left(-U x-\frac{\sigma}{M} \eta\right) \Delta \theta_{2} .
\end{aligned}
$$

To obtain a dynamic condition at the interface, Chuoke et al. (1959) assumed that the pressure jump across the interface in a porous medium can be expressed in terms of a macroscopic Laplace formula

$$
p_{1}-p_{2}=T *\left(\frac{\partial^{2} \eta}{\partial x *^{2}}+\frac{\partial^{2} \eta}{\partial y *^{2}}\right)+p_{c}(t)
$$

where $T^{*}$ is an effective (macroscopic) surface tension coefficient and $p_{c}(t)$ is related to capillary pressure drops across microscopic interfaces but does not depend on the curvature of the macroscopic interface, only on time. The concept of $T^{*}$ is crucial to the analysis and we present a separate discussion of it at the end of this section.

When Darcy's law holds, the velocity potential takes the form

$$
\phi_{j}=\frac{k_{j}}{\mu_{j}}\left[p_{j}+\rho_{j} g(x * \cos \alpha+y * \cos \beta)+f_{j}(t)\right]
$$

where $f_{j}(t)$ is an arbitrary function of time. Substituting (3.43) and (3.44) into (3.46), and solving for $p_{j}(t)$, yields

$$
\begin{aligned}
& p_{1}=\frac{\mu_{1} \Delta \theta_{1}}{k_{1}}\left(-U x+\frac{\sigma}{M} \eta\right)-\rho_{1} g(x * \cos \alpha+\eta \cos \beta)-f_{1}(t) \\
& p_{2}=\frac{\mu_{2} \Delta \theta_{2}}{k_{2}}\left(-U x-\frac{\sigma}{M} \eta\right)-\rho_{2} g(x * \cos \alpha+\eta \cos \beta)-f_{2}(t) .
\end{aligned}
$$


Substituting (3.38), (3.39), (3.47) and (3.48) into (3.45) leads to

$$
f_{2}(t)-f_{1}(t)=p_{c}(t)
$$

and the relationships

$$
\begin{gathered}
\left(\frac{\mu_{1} \Delta \theta_{1}}{k_{1}}+\frac{\mu_{2} \Delta \theta_{2}}{k_{2}}\right) \frac{\sigma}{M}=\left(\frac{\mu_{1} \Delta \theta_{1}}{k_{1}}-\frac{\mu_{2} \Delta \theta_{2}}{k_{2}}\right) U \cos \varphi_{2}+\left(\rho_{1}-\rho_{2}\right) g \cos \beta-T^{*} M^{2} \\
\left(\frac{\mu_{1} \Delta \theta_{1}}{k_{1}}-\frac{\mu_{2} \Delta \theta_{2}}{k_{2}}\right) U \cos \varphi_{1}+\left(\rho_{1}-\rho_{2}\right) g \cos \alpha=0 \\
\left(\frac{\mu_{1} \Delta \theta_{1}}{k_{1}}-\frac{\mu_{2} \Delta \theta_{2}}{k_{2}}\right) U \cos \varphi_{3}=0 .
\end{gathered}
$$

From (3.52) one finds that, as long as $\Delta \theta_{1} \mu_{1} / k_{1} \neq \Delta \theta_{2} \mu_{2} / k_{2}, \cos \varphi_{3}$ must be zero. Hence $x$ and $z^{*}$ are perpendicular to each other and $z$ coincides with $z^{*}$. This means that $(\mathrm{x}, \mathrm{y})$ and $\left(x^{*}, y^{*}\right)$ share a vertical plane and the problem becomes two-dimensional. Then the angle between $x$ and $y^{*}, \varphi_{2}=\pi / 2-\varphi_{1}$, is determined by (3.51). For the interface to be orthogonal to the velocity ( $\left.\cos \varphi_{1}=0\right), \cos \alpha$ must generally be equal to zero and the flow must be vertical. In all other cases, the interface is inclined to the direction of flow at an angle other than $90^{\circ}$. This is contrary to the premise of Chuoke et al. (1959).

From (3.50), the conditions for unstable flow to develop in three-dimensional porous media are

$$
\left(\frac{\mu_{1} \Delta \theta_{1}}{k_{1}}-\frac{\mu_{2} \Delta \theta_{2}}{k_{2}}\right) U \cos \varphi_{2}+\left(\rho_{1}-\rho_{2}\right) g \cos \beta>0
$$

and

$$
M^{2}<M_{c}^{2}=\frac{1}{T^{*}}\left[\left(\frac{\mu_{1} \Delta \theta_{1}}{k_{1}}-\frac{\mu_{2} \Delta \theta_{2}}{k_{2}}\right) U \cos \varphi_{2}+\left(\rho_{1}-\rho_{2}\right) g \cos \beta\right] .
$$

The corresponding instability criteria by Chuoke et al. (1959) do not contain $\cos \varphi_{2}$ and therefore hold only for vertical flow. This is so despite the fact that their derivation is cast in terms of a volumetric flux $U$ which is said to have an arbitrary orientation.

By taking the derivative of $\sigma$ with respect to $M$ in equation (3.50) and setting it 
equal to zero, one finds that the fastest growing harmonic is that with wave number

$$
M_{m}=M_{c} / \sqrt{3} \text {. }
$$

If we define a wavelength $\lambda$ as $2 \pi / M$, then $1 / \lambda^{2}=1 / \lambda_{x}{ }^{2}+1 / \lambda_{y}{ }^{2}$ where $\lambda_{x}=2 \pi / n$ and $\lambda_{y}=$ $2 \pi / m$ are wavelengths in the $x$ and $y$ directions. From (3.54) and (3.55) it follows that

$$
\lambda_{m}=\sqrt{3} \lambda_{c}=2 \sqrt{3} \pi\left[\frac{T^{*}}{\left(\Delta \theta_{1} \mu_{1} / k_{1}-\Delta \theta_{2} \mu_{2} / k_{2}\right) U \cos \varphi_{2}+\left(\rho_{1}-\rho_{2}\right) g \cos \beta}\right]^{1 / 2}
$$

where $\lambda_{m}$ and $\lambda_{c}$ are wavelengths corresponding to the fastest and critical harmonics, respectively. In the three-dimensional case, neither $\lambda_{x}$ nor $\lambda_{y}$ can generally be specified. If one assumes that $\lambda_{x}=\lambda_{y}$ then $\lambda_{x}=\lambda_{y}=\sqrt{2} \lambda_{3}$ and the most unstable wavelengths are given by

$$
\lambda_{x m}=\lambda_{y m}=\sqrt{2} \lambda_{m}=2 \sqrt{6} \pi\left[\frac{T^{*}}{\left(\Delta \theta_{1} \mu_{1} / k_{1}-\Delta \theta_{2} \mu_{2} / k_{2}\right) U \cos \varphi_{2}+\left(\rho_{1}-\rho_{2}\right) g \cos \beta}\right]^{1 / 2} .
$$

In the special case where water is displacing air downward, the latter reduces to

$$
\lambda_{x m}=\lambda_{y m}=\sqrt{2} \lambda_{m}=2 \sqrt{6} \pi\left(\frac{T^{*}}{\rho_{2} g-\Delta \theta_{2} \mu_{2} U / k_{2}}\right)^{1 / 2} .
$$

For two-dimensional porous media, the stability criteria are similar to those for Hele-Shaw cells upon replacing $T$ by $T^{*}, \mu_{1} / k_{1}$ by $\Delta \theta_{1} \mu_{1} / k_{1}$, and $\mu_{2} / k_{2}$ by $\Delta \theta_{2} \mu_{2} / k_{2}$. For example, the most unstable wavelength for water displacing air downward in 2-D is, in analogy to (3.37),

$$
\lambda_{m}=2 \sqrt{3} \pi\left(\frac{T *}{\rho_{2} g-\Delta \theta_{2} \mu_{2} U / k_{2}}\right)^{1 / 2} .
$$

This differs from (3.58) by a factor of $\sqrt{2}$, suggesting that fingers in 3-D are wider along at least one transverse direction than are their counterparts in 2-D.

Chuoke et al. (1959) based their concept of effective surface tension $T^{*}$ on heuristic energy arguments. Homsy (1987) questioned the validity of these arguments without explanation. By matching wavelengths predicted by their theory to measured wavelengths during the displacement of oil by water in a medium of glass beads, Chuoke et al. (1959) determined that $T^{*}=C^{*} T$ where $C^{*}=7.6$. Similar laboratory experiments on 
displacement of oil by water led Peters and Flock (1981) to $C^{*}$ values ranging from 5.45 in oil-wet porous media to 306.25 in water-wet porous media. White et al. (1976) proposed the relationship

$$
T^{*}=T /\left(\theta_{s}-\theta_{i}\right)
$$

for infiltration of water into unsaturated soils where $\theta_{s}$ is volumetric water content behind the wetting front and $\theta_{i}$ is initial water content ahead of the wetting front. They conjectured that projections of microscopic menisci on the macroscopic interface occupy a fraction $\left(\theta_{s}-\theta_{i}\right)$ of this interface. In other words, the actual perturbed interface has curvatures which, on the average, exceed by $\left(\theta_{s}-\theta_{i}\right)^{-1}$ those of the macroscopic wetting front.

Recently, Chang et al. (1994) proposed to view the interface as fractal. In their view, the surface tension force $F$ acting along an interface in a porous medium may be written as

$$
F=T B^{*}=T^{*} B
$$

where $B$ and $B^{*}$ are the lengths of the macroscopic and microscopic interfaces, respectively. From considerations of fractal geometry (Mandelbrot, 1982) it follows that

$$
B^{*}=a d^{1-D}
$$

where $a$ is a constant, $\mathrm{d}$ is an experimental length scale, and $D$ is fractal dimension. The parameters $a$ and $D$ are obtained from a $\log \log$ plot of $B^{*}$ measured on the laboratory scale and the resolution $d$ of the measuring device (the length of a rigid ruler used to measure $B^{*}$ ). Equation (3.62) is then assumed to hold at the unobserved microscopic scale. Combining (3.61) and (3.62) gives the scaling relation

$$
T^{*}=\frac{a d^{1-D}}{B} T .
$$

To computer $T^{*}$ the authors set $B$ equal to the width of the experimental chamber and $d$ to the mean pore size. Upon introducing the result into (3.59), the authors found the computed dominant unstable wavelengths to agree well with experimental results at early time but not at later time. The lack of agreement at late time might have stemmed from the linear nature of the stability analysis that had led to (3.59). 


\subsection{Wetting Front Instability}

In this report, we are interested mostly in the stability of wetting fronts during water infiltration into unsaturated media. We therefore present below selected analyses of this problem.

\subsubsection{Analysis of Raats (1973)}

Over twenty years ago, Raats (1973) presented an analysis of wetting front instability based on the Green and Ampt (1911) model. He conjectured that small perturbations in an initially planar wetting front will tend to grow if the front velocity increases with depth, and will tend to disappear if it decreases with depth. The Green and Ampt model treats the front as a sharp boundary between two regions of uniform water content, one above and one below. It provides a reasonably good approximation for wetting fronts that are not too diffuse.

Consider a sharp wetting front at $z=-L$ where $L$ is depth and $z$ is a vertical coordinate with origin at the soil surface, positive upward. The hydraulic conductivity and volumetric moisture content in the wetter region above the front are $K_{s}(z)$ and $\theta_{s}(z)$, and in the dryer region below the front are $K_{i}(z)$ and $\theta_{i}(z)$. The volumetric flux above the front is

$$
q=-K_{s}(z) \frac{\partial h}{\partial z} \quad ; \quad z \geq-L
$$

where $h$ is total hydraulic head. In stable flows $q$ varies with time but not with elevation. Head at the soil surface and at the front is given by

$$
h=\psi_{o} \text { at } z=0 ; \quad h=\psi_{a}+\psi_{f}-L \text { at } z=-L
$$

where $\psi_{0}$ is pressure head in the water at the soil surface (taking atmospheric pressure to be zero), $\psi_{a}$ is pressure head due to increased air pressure at $z=-L$ relative to that at the surface, and $\psi_{f}$ is capillary pressure head in the water at the interface. Integrating (3.64) between the limits (3.65) gives

$$
q=-K_{s} \frac{\partial h}{\partial z}=-\frac{\psi_{a}+\psi_{f}-L-\psi_{o}}{\int_{0}^{-L} \frac{1}{K_{s}(z)} d z} .
$$

Defining a new (depth) variable $\xi=-z$ allows rewriting this as 


$$
q=\frac{\psi_{a}+\psi_{f}-\psi_{o}-L}{\int_{0}^{L} \frac{1}{K_{s}(\xi)} d \xi}
$$

If flow below the front is negligible then the velocity of the front, $U$, is

$$
U=\frac{q}{\Delta \theta}=\frac{\psi_{a}+\psi_{f}-\psi_{o}-L}{\Delta \theta \int_{0}^{L} \frac{1}{K_{s}(\xi)} d \xi}
$$

where $\Delta \theta=\theta_{s}-\theta_{i}$. Since $U$ is negative, Raats' conjecture implies that the wetting front is unstable whenever $\mathrm{d} U / \mathrm{d} L<0$. We consider below some special forms of this instability criterion.

In uniform media $\psi_{f}$ (e.g., Neuman, 1976), $\Delta \theta$, and $K_{s}$ are independent of $L$ and (3.68) simplifies to

$$
U=\frac{K_{s}}{\Delta \theta}\left(\frac{\psi_{a}+\psi_{f}-\psi_{o}}{L}-1\right)
$$

Assuming that $\psi_{o}$ and $\psi_{a}$ are independent of $L$, differentiating (3.69) with respect to $L$ gives

$$
\frac{d U}{d L}=-\frac{K_{s}}{L^{2} \Delta \theta}\left(\psi_{a}+\psi_{f}-\psi_{o}\right) .
$$

The factor $K_{s} / L^{2} \Delta \theta$ is inherently positive. It thus follows that the front is unstable whenever

$$
\psi_{a}+\psi_{f}-\psi_{o}>0
$$

This happens whenever pressure head at the surface $\left(\psi_{o}\right)$ is smaller than that at the wetting front $\left(\psi_{a}+\psi_{f}\right)$, a situation that can arise only if air pressure below the wetting front exceeds atmospheric. This is so because $\psi_{f}$ is always smaller (more negative) than $\psi_{o}$ (ibid). However, in water repellent soils $\psi_{f}$ may be positive, allowing the wetting front to be unstable even if air pressure below the wetting front does not exceed atmospheric.

If $K_{s}$ is a function of depth while $\psi_{f}, \psi_{o}, \psi_{a}$, and $\Delta \theta$ are constant, differentiating (3.68) with respect to $L$ and setting $\mathrm{d} U / \mathrm{d} L<0$ yields 


$$
K_{s}(L) \int_{0}^{L} \frac{1}{K_{s}(\xi)} d \xi-L>-\left(\psi_{a}+\psi_{f}-\psi_{o}\right)
$$

If $K_{s}$ increases with depth, so does the left hand side of (3.72), and the front becomes unstable when it reaches a critical depth (obtained upon replacing $>$ by $=$ in the above equation). If $K_{s}$ decreases with depth, so does the left hand side of (3.72), rendering the front stable. Raats (1973) used similar arguments to conclude that a decrease in $\psi_{f}$ and/or an increase in initial water content with depth have destabilizing effects on the wetting front.

Consider two uniform layers, the top of thickness $L_{t}$ and hydraulic conductivity $K_{t}$. Then, for $L>L_{t},(3.68)$ yields

$$
U=\frac{\psi_{a}+\psi_{f}-\psi_{o}-L}{\Delta \theta\left[L_{t} / K_{t}+\left(L-L_{t}\right) / K_{b}\right]}
$$

where $K_{b}$ is the hydraulic conductivity of the bottom layer. Here again $\psi_{f}$ and $\Delta \theta$ are independent of $L$. If the same is true for $\psi_{a}$ and $\psi_{o}$ then the flow becomes unstable whenever

$$
K_{b}>K_{t}\left(-\frac{\psi_{a}+\psi_{f}-\psi_{o}}{L_{t}}+1\right)
$$

The right hand side of (3.74) is the flux just as the wetting front enters the bottom layer. Since (3.74) is independent of $L$, the wetting front becomes unstable immediately upon crossing the interface between the layers.

\subsubsection{Analysis of Philip (1975)}

Raats' (1973) conjecture that a wetting front becomes unstable if its velocity increases with depth was based on intuition. His analysis was extended to incorporate surface tension, and placed on a more rigorous footing, by Philip (1975). The problem is defined as in the previous analysis by Raats. It can then be shown (Appendix A) that the gradient $G$ of pressure head immediately above the wetting front is

$$
G=-\frac{\psi_{a}+\psi_{f}-\psi_{o}-L}{K_{s}(L) \int_{0}^{L} \frac{1}{K_{s}(\xi)} d \xi}-1
$$


where $K_{s}$ is a function of $\xi=-z$. Next, let the horizontal wetting front $z=-L(t)$ at time $t_{o}$ be perturbed slightly into a three dimensional wave form, and write a single harmonic of this wave as

$$
z=-L+\Theta=-L+\varepsilon(t) \sin (n x+\tau) \sin (m y+\delta)
$$

Here $\Theta$ is the perturbation, $\varepsilon$ is a small positive amplitude, $n$ and $m$ are wave numbers corresponding to $x$ and $y$, respectively, and $\tau$ and $\delta$ are corresponding phase shifts. Clearly, the perturbation $\Theta$ grows with time (the front is unstable) if $d \varepsilon / d t>0$, and decays with time (the front is stable) if $d \varepsilon / d t<0$. It is shown in Appendix A that

$$
\varepsilon(t)=\varepsilon_{o} \exp \left(\int_{t_{o}}^{t} \vartheta d t\right)
$$

where, including a modification proposed by White et al. (1976),

$$
\vartheta=-\frac{K_{s}(-L)}{\Delta \theta}\left(G+\frac{M^{2} T}{\Delta \theta \rho g}\right) \zeta .
$$

Here $M^{2}=n^{2}+m^{2}, \Delta \theta=\theta_{s}-\theta_{i}, \varepsilon_{o}$ is a positive constant, and $\zeta=-d \Omega / d z$ at $z=-L$ where $\Omega$ satisfies

$$
\frac{1}{K_{s}} \frac{d}{d z}\left(K_{s} \frac{d \Omega}{d z}\right)-M^{2} \Omega=0
$$

subject to

$$
z=0, \Omega=0 ; z=-L, \Omega=1 \text {. }
$$

As explained in Appendix A, $\zeta$ is positive for $K_{s}>0$.

Since $d \varepsilon / d t=\vartheta(t) \varepsilon(t)$, it is clear that the front is unstable when $\vartheta>0$ and stable otherwise. As $K_{s}, \Delta \theta, M^{2}, T$ and $\zeta$ are inherently positive, a necessary criterion for instability is

or

$$
G<0
$$

In other words, instability can develop only if the pressure gradient immediately above the front is negative, i.e., the pressure head increases downward. When the pressure head is 
uniform immediately above the wetting front or increases upward, the front remains stable. Criterion (3.82) is identical to (3.72) due to Raats (1973). When $K_{s}$ is constant, it reduces to (3.71) and, for a two-layered profile, to (3.74) as proposed by Raats.

A sufficient condition for instability is

$$
M<M_{c}=\left(-\frac{\Delta \theta \rho g G}{T}\right)^{1 / 2} .
$$

This means that, for a given negative $G$, only disturbances with $M$ less than the critical value $M_{c}$ can produce instability. To determine the most unstable disturbance, $M_{m}$, Philip used the approximation

$$
\zeta \propto M
$$

which he had shown holds when $M$ is large compared with the reciprocal of $L . M_{m}$ corresponds to maximum $\vartheta$ at $d \vartheta / d M=0$, or

$$
M_{m}=\frac{M_{c}}{\sqrt{3}}
$$

For a wavelength $\lambda=2 \pi / M$ this yields

$$
\lambda_{c}=\frac{2 \pi}{M_{c}}=2 \pi\left(-\frac{T}{\Delta \theta \rho g G}\right)^{1 / 2}
$$

and

$$
\lambda_{m}=\frac{2 \pi}{M_{m}}=\sqrt{3} \lambda_{c}=2 \sqrt{3} \pi\left(-\frac{T}{\Delta \theta \rho g G}\right)^{1 / 2}
$$

where $\lambda_{c}$ and $\lambda_{m}$ are wavelengths corresponding to the critical and fastest harmonics, respectively. As discussed in Section 3.2, wave numbers $n$ and $m$ corresponding to critical and most unstable harmonics cannot be generally determined. Under the assumption that $n=m$, we have $n=m=M / \sqrt{2}$. The wavelengths $\lambda_{x}(2 \pi / n)$ and $\lambda_{y}(2 \pi / m)$ in $x$ and $y$ directions, respectively, are then both equal to $\sqrt{2} \lambda$. The most unstable wavelengths in the $x$ or $y$ directions are

$$
\lambda_{x m}=\lambda_{y m}=\sqrt{2} \lambda_{m}=2 \sqrt{6} \pi\left(-\frac{T}{\Delta \theta \rho g G}\right)^{1 / 2} .
$$


Philip noted that for redistribution to occur following the cessation of infiltration, the Green and Ampt model requires that air invade the advancing water column from above (at the soil surface) and/or below (across the wetting front). Air entry across the wetting front would require that part of the front retreat upward. Due to capillary hysteresis, air entry (upward) would require an unlikely reduction in pressure head at the wetting front. It is therefore much more likely that redistribution would occur as a result of air entry at the soil surface. This would entail a reduction in pressure head at the surface from $\psi_{0}$ to some negative air entry value $\psi_{\text {entry }}$. When $\psi_{a}=0,(3.82)$ then implies that, in a uniform medium, the front becomes unstable whenever

$$
\psi_{\text {entry }}<\psi_{f}
$$

i.e., whenever the air-entry pressure head is more negative than the pressure head at the wetting front.

For a uniform soil, the analysis of Philip (1975) is in complete agreement with that of Chuoke et al. (1959). Under vertical infiltration of water into an initially unsaturated soil $\left(\beta=\pi, \varphi_{2}=0, \rho_{2} \gg \rho_{1}\right.$, and $\left.\mu_{2} \gg \mu_{1}\right)$, criteria (3.53) and (3.54) due to Chuoke et al. reduce to

$$
\begin{gathered}
-\frac{\mu_{2}}{k_{2}} U \Delta \theta_{2}+\rho_{2} g>0 \\
M^{2}<M_{c}^{2}=\frac{1}{T *}\left(-\frac{\mu_{2}}{k_{2}} U \Delta \theta_{2}+\rho_{2} g\right)
\end{gathered}
$$

where the numerical subscript 2 is retained for consistency with (3.53) and (3.54). Here the vertical coordinate is positive downward, so Darcy's law is written as

$$
U \Delta \theta_{2}=\frac{k_{2}}{\mu_{2}} \rho_{2} g(G+1)
$$

Substituting this into (3.90) and (3.91) yields

$$
\begin{gathered}
G<0 \\
M<M_{c}=\left(-\frac{\rho_{2} g G}{T^{*}}\right)^{1 / 2} .
\end{gathered}
$$

Setting $T^{*}=T / \Delta \theta$ renders (3.93) and (3.94) identical with (3.81) and (3.83) due to Philip 
(1975). The most unstable wavelength during immiscible displacement according to (3.56) and (3.92) is

$$
\lambda_{m}=2 \sqrt{3} \pi\left(-\frac{T^{*}}{\rho_{2} g G}\right)^{1 / 2}
$$

the same as (3.87) according to Philip. As the latter was obtained by assuming that $\zeta \propto$ $M$, we may conclude that this assumption was reasonable, at least for uniform media.

\subsubsection{Analyses of Parlange and Hill (1976) and Glass et al. (1989a, 1991)}

The concept of effective surface tension is not based on a rigorous analysis and $T^{*}$ cannot generally be determined by measurement. Parlange and Hill (1976) suggested that in porous media where macroscopic fluid-fluid interfaces are generally not sharp, it is more appropriate to write a mass balance equation which incorporates diffusion induced by capillary forces. Instead of a pressure jump across the interface, they introduced a formulation in which the movement of a convex front is slowed down in proportion to its curvature. The authors assumed that if $U$ is the velocity of a flat front, and $v$ the velocity of a curved front, then the two are related via

$$
v=U-\Gamma\left(r_{1}^{-1}+r_{2}^{-1}\right)
$$

where $\Gamma$ is a coefficient and $r_{1}^{-1}$ and $r_{2}^{-1}$ are principal curvatures of the interface, taken to be positive when the center of curvature is in the water behind the front. In a manner analogous to the work of Markstein (1951) on flame instability, Parlange and Hill found that $\Gamma$ along two- and three-dimensional fronts is given by (Appendix $B$ )

$$
\Gamma=\frac{S^{2}}{2(\Delta \theta)^{2}}
$$

where $\Delta \theta=\theta_{s}-\theta_{i}, S$ is sorptivity and can be approximated by (Appendix B)

$$
S^{2} \approx 2 \Delta \theta \int_{\theta_{i}}^{\theta_{s}} D d \theta,
$$

and $\mathrm{D}$ is soil-water diffusivity.

The following two-dimensional analysis due to Parlange and Hill (1976) is similar to that of Saffman and Taylor (1958) but uses (3.96) instead of (3.17). The front at time $t$ 
$=0$ is perturbed slightly into a wave form. A single harmonic of this wave can be described by

$$
z=\eta(x, t)=\varepsilon \mathrm{e}^{i n x+\sigma t}
$$

where $z$ is a vertical coordinate taken positive downward with origin at the unperturbed front, $x$ is the horizontal axis, $i=\sqrt{-1}, n$ is wave number or period, $\varepsilon$ is a small positive constant, and $\sigma$ is amplification or growth factor. The authors write hydraulic head $h$ behind the wetting front as

$$
h=\frac{p}{\rho g}-z
$$

and take them to satisfy the Laplace equation,

$$
\nabla^{2} h=0 \text {. }
$$

The kinematic condition at the interface, $\mathrm{D}(z-\eta) / \mathrm{D} t=0$ at $z=\eta$ where $\mathrm{D}$ denotes hydrodynamic differential, can be expressed to a first order of approximation as

$$
-\frac{K_{s}}{\Delta \theta} \frac{\partial h}{\partial z}=U+\sigma \varepsilon \mathrm{e}^{i n x+\sigma t}
$$

where the term on the left represents front velocity, the first term on the right represents the mean velocity of the front, and the second term on the right represents the rate $\partial \eta / \partial t$ at which perturbations $z=\eta$ in (3.99) grow or decay with time. The authors write

$$
-\frac{K_{s}}{\Delta \theta} \frac{\partial h}{\partial z}=v+\sigma \varepsilon \mathrm{e}^{i n x+\sigma t}
$$

instead of (3.102a) and deduce that

$$
v=U+\varepsilon n^{2} \Gamma \mathrm{e}^{i n x+\sigma t}
$$

We shall return to (3.102b) and (3.103) later. Solutions of (3.101) subject to (3.102b), (3.103) and the stipulation that perturbations decay far behind the front, at infinity, $z=-$ $\infty$, take the form

$$
-\frac{K_{s}}{\Delta \theta} h=U z+\left(\frac{\sigma}{n}+\Gamma n\right) \varepsilon \mathrm{e}^{i n x+n z+\sigma t} .
$$

At the interface where $z=\eta$, (3.104) can be approximated to first order by 


$$
-\frac{K_{s}}{\Delta \theta} h=U \eta+\left(\frac{\sigma}{n}+\Gamma n\right) \eta
$$

Next, Parlange and Hill (1976) take pressure to be continuous across the interface and set it equal to atmospheric, or zero. With this and (3.100), (3.105) can be rearranged to yield

$$
\sigma=\left(\frac{K_{s}}{\Delta \theta}-U\right) n-\Gamma n^{2}
$$

This implies that, for instability to occur, it is necessary that

$$
\frac{K_{s}}{\Delta \theta}-U>0 \quad \text { or } \quad q=\Delta \theta U<K_{s} .
$$

In other words, a front is unstable only if its flux $q$ is smaller than the hydraulic conductivity behind it. Note that, according to Darcy's law,

$$
q=K_{s}(G+1) .
$$

Hence (3.107) is the same as the instability criterion (3.81) due to Philip (1975), $G<0$. The critical wave number and wavelength are

$$
n_{c}=\frac{1}{\Gamma}\left(\frac{K_{s}}{\Delta \theta}-U\right) \quad \lambda_{c}=2 \pi \frac{\Gamma \Delta \theta}{K_{s}-U \Delta \theta}
$$

The most unstable wave number and wavelength are those corresponding to $d \sigma / d n=0$, or

$$
n_{m}=\frac{n_{c}}{2}=\frac{1}{2 \Gamma}\left(\frac{K_{s}}{\Delta \theta}-U\right) \quad \lambda_{m}=2 \lambda_{c}=4 \pi \frac{\Gamma \Delta \theta}{K_{s}-U \Delta \theta}
$$

The finding here that the most unstable wavelength is twice the critical wavelength differs from the findings of Saffman and Taylor (1958; and Neuman, 1982), Chuoke et al. (1959), and Philip (1975) that $\lambda_{m}$ is $\sqrt{3}$ times the critical wavelength.

We were unable to verify (3.102b) and (3.103). We don't understand the rationale for writing (3.102b) instead of (3.102a). Likewise, as $z$ is vertical and positive downward, and $r_{1}^{-1}$ is positive when the center of curvature is in the water, one finds from (3.99) and the definition of curvature that 


$$
r_{1}^{-1}=-\frac{\partial^{2} \eta}{\partial x^{2}}=\varepsilon n^{2} e^{i n x+\sigma t}
$$

$\left(r_{2}^{-1}\right.$ is zero in the 2-D case). Substituting (3.111) into (3.96) one obtains

$$
v=U-\varepsilon n^{2} \Gamma e^{i n x+\sigma t}
$$

which differs from (3.103) by a sign. To recover consistency with the instability criterion (3.81) due to Philip (1975) and hence with (3.107), it would appear necessary to replace the minus sign in (3.96) by a plus sign. This, however, would invalidate Appendix B as well as (3.97) according to which $\Gamma$ is positive.

Glass et al. (1991) extended the analysis of Parlange and Hill (1976) to three dimensions. They found the most unstable wavelength to be

$$
\lambda_{m}=19.2 \frac{\Gamma \Delta \theta}{K_{s}-U \Delta \theta}
$$

The ratio 1.53 between the three-dimensional factor 19.2 and the two-dimensional factor $4 \pi$ is somewhat larger than the ratio $\sqrt{2}=1.42$ in works we cited previously.

It is often stated (e.g., Chuoke et al., 1959; Glass et al., 1989a) on the basis of firstorder stability analyses that finger width, or diameter, are half the most unstable wavelength. In general, there is no guarantee that the initial disturbance will develop into a dominant finger and persist in that role. Based on a dimensional analysis, Glass et al. (1989a) proposed

$$
d=\frac{\Gamma \Delta \theta}{K_{s}} f\left(q / K_{s}\right)
$$

where $d=\lambda_{m} / 2$ is finger width and $f(\cdot)$ is some function. A comparison between (3.114) and (3.110) reveals that, in two dimensions, $f\left(q / K_{s}\right)=2 \pi /\left(1-q / K_{s}\right)$. This has been verified experimentally by Glass et al. (1989b).

Equations (3.114) suggests that finger width is proportional to $\Gamma \Delta \theta$ and inversely proportional to $K_{s}-q$. One should therefore expect fingers to widen as $q$ increases for given $\Gamma, \Delta \theta$, and $K_{s}$; this has indeed been observed by Glass et al. (1989b). In contrast, the experiments of Hill and Parlange (1972) showed that changing the flow rate merely changes the number of fingers, but not their widths. As mentioned earlier, Glass et al. 
(1989b) attributed this difference to soil heterogeneity in the experiments of Hill and Parlange.

\subsubsection{Analysis of Diment et al. (1982)}

In all previous analyses, the front was considered either to be sharp or to propagate at the same rate as a sharp boundary. Diment et al. (1982) attempted to analyze the stability of fronts controlled by the two-dimensional Richards equation of unsaturated flow,

$$
\frac{\partial \theta}{\partial t}=\frac{\partial}{\partial x}\left[K(\psi) \frac{\partial \psi}{\partial x}\right]+\frac{\partial}{\partial z}\left[K(\psi)\left(\frac{\partial \psi}{\partial z}+1\right)\right]
$$

where $\psi$ is pressure head, $\theta$ is volumetric water content, $x$ is horizontal coordinate, and $z$ is vertical coordinate with positive upward. They wrote

$$
\psi(x, z, t)=\psi_{m}(z, t)+\varepsilon \psi^{\prime}(x, z, t)
$$

where $\psi_{m}$ is pressure head in the unperturbed field (assumed to vary only vertically), $\varepsilon$ is a small positive constant, and $\psi^{\prime}$ is a perturbation in pressure head. They then set

$$
\psi^{\prime}(x, z, t)=\mathrm{e}^{i n x+\sigma t} f(z)
$$

where $f(z)$ is some function of $z$. In their analysis, changes in $\psi$ were allowed to cause changes in $K$ and $\theta$ according to

$$
K(\psi)=K_{m}(\psi)+\varepsilon K^{\prime}(\psi) \quad \text { and } \quad \theta(\psi)=\theta_{m}(\psi)+\varepsilon \theta^{\prime}(\psi)
$$

where again $m$ designates unperturbed states and the prime defines perturbations. To simplify the analysis the authors assumed that

$$
K^{\prime}(\psi)=\frac{d K_{m}}{d \psi} \psi^{\prime} \quad \text { and } \quad \theta^{\prime}(\psi)=\frac{d \theta_{m}}{d \psi} \psi^{\prime} .
$$

Both perturbed $(\psi, K, \theta)$ and unperturbed $\left(\psi_{m}, K_{m}, \theta_{m}\right)$ quantities satisfy (3.115). A corresponding equation for the perturbations $\left(\psi^{\prime}, K^{\prime}, \theta^{\prime}\right)$ can be obtained by subtracting the unperturbed equation from the perturbed equation. Upon transforming the resulting perturbation equation so that the vertical coordinate moves with the average velocity of the front, $\bar{v}$, and neglecting the cross-derivative $\partial^{2} \theta_{m} / \partial \psi \partial t$, Diment et al. obtained 


$$
A_{m} \frac{d^{2} f}{d z^{2}}+B_{m} \frac{d f}{d z}+C_{m} f=\sigma f
$$

where $A_{m}=K_{m} / \theta_{m}$

$$
\begin{aligned}
& B_{m}=\left[\frac{\partial K_{m}}{\partial z}+\frac{\partial K_{m}}{\partial \psi}\left(\frac{\partial \psi_{m}}{\partial z}+1\right)-\bar{v} \frac{\partial \theta_{m}}{\partial \psi}\right] / \frac{\partial \theta_{m}}{\partial \psi} \\
& C_{m}=\left[-n^{2} K_{m}+\frac{\partial^{2} K_{m}}{\partial \psi \partial z}\left(\frac{\partial \psi_{m}}{\partial z}+1\right)+\frac{\partial K_{m}}{\partial \psi} \frac{\partial^{2} \psi_{m}}{\partial z^{2}}-\bar{v} \frac{\partial^{2} \theta_{m}}{\partial \psi \partial z}\right] / \frac{\partial \theta_{m}}{\partial \psi} .
\end{aligned}
$$

There is no known analytical solution for (3.120) - (3.123). The authors solved it by finite differences subject to the requirement that there be no perturbation at the upper and lower boundaries. This led to a system of coupled nonlinear algebraic equations

$$
(\mathbf{R}-\sigma \mathbf{I}) \mathbf{f}=0
$$

where $\mathbf{R}$ is a tridiagonal matrix, $\mathbf{I}$ is the identity matrix, and $\mathbf{f}$ is a vector of $f$ values at the nodes. For nontrivial $f(z), \operatorname{det}(\mathbf{R}-\sigma \mathrm{I})=0$. Hence the problem reduces to that of finding the eigenvalues of the matrix $\mathbf{R}$. If all eigenvalues are nonpositive, the flow is unconditionally stable. If any eigenvalue is positive, it may cause instability under the assumed conditions.

Numerical results were given by Diment and Watson (1983) for several cases including redistribution following infiltration, infiltration into a heterogeneous medium in which hydraulic conductivity increases with depth, and infiltration into a fine-over-coarse two-layered system. In no case was the actual flow problem solved; only the eigenvalues of $\mathbf{R}$ were determined. All cases assumed a uniform initial water content of 0.05 and ignored hysteresis. Although one expects instability to develop in several of these cases, in none has it been predicted by the above method. The authors concluded that minor amounts of antecedent moisture may help eliminate instabilities that could, in soils which are initially dry, develop into fingers. Though their numerical scheme was inadequate to test this conclusion, they were able to corroborate it experimentally (Diment and Watson, 1985).

\subsubsection{Analysis of Hillel and Baker (1988)}

As mentioned briefly in Chapter 2, Hillel and Baker (1988) had advanced an etiologic hypothesis to explain the development of fingers during infiltration into layered 
soils. They criticized previously published theories of wetting front instability as being formalistic rather physically based, and regarded their theory as direct, mechanistic, and applicable to both sharp and diffuse wetting fronts.

The fundamental hypothesis of Hillel and Baker (1988) is similar to that of Raats (1973), namely, that the cross-sectional area available for flow diminishes as a front accelerates downward. Their explanation considers infiltration into an initially dry layer of soil which is less conductive than the underlying layer. During early ponded infiltration a stable horizontal wetting front develops in the top-layer. When the bottom edge of this diffuse front hits the interface between the layers, suction within it is too high to allow entry into the coarser sublayer. The front therefore pauses temporarily until water content behind it builds up, and suction diminishes, to a sufficient extent that breakthrough becomes possible. Early breakthrough takes place into the smallest pores in the sublayer which are distributed randomly over the area of the interface. These pores admit only a portion of the water accumulated above the soil interface, allowing suction there to continue falling. As it does so, larger and larger pores near the random breakthrough locations take part in the breakthrough. The random breakthrough areas widen until they become capable of conducting all the water that the top layer can supply. The corresponding suction, $\psi_{e}$, is referred to as effective water entry suction by the authors. If the conductivity of the sublayer, $K_{u}$, at its effective water entry suction exceeds the constant flux through the toplayer, $Q_{t}$,

$$
K_{u}\left(\psi_{e}\right)>Q_{t},
$$

then only a fraction of the sublayer conducts the water delivered to it from above. This results in the formation of fingers. The fraction, $F$, of the sublayer occupied by fingers is simply

$$
\mathrm{F}=\frac{Q_{t}}{K_{u}\left(\psi_{e}\right)}
$$

One expects the effective water entry suction to depend on conditions in the top layer. However, experiments by Baker and Hillel $(1990,1991)$ show that it depends mainly on the properties of the sublayer, at least for the case of two uniform layers with narrow pore size distributions that are initially dry. The authors found that, for such layers, the effective water entry suction is near or at the inflection point in the $\psi-\theta$ curve and not near saturation. No general conclusions were drawn about $\psi_{e}$ under other conditions. 


\section{NUMERICAL SIMULATIONS}

Virtually all the analytical developments in Chapter 3 represented first-order studies concerning the onset of instability. As fingers grow, their behavior becomes strongly nonlinear and, as a rule, requires numerical analysis. In this chapter, we discuss briefly some numerical methods which have been used in the literature to study the phenomenon.

\subsection{Vortex Methods}

During immiscible displacement of two incompressible fluids in a Hele-Shaw cell, the bulk of the flow field is irrotational and vortices are restricted to the interface (Meiburg and Homsy, 1988a). Since the interface is very thin compared to its length, the region of non-zero vorticity can be described as a vortex sheet. As will be seen later, knowing the vorticity field allows determining the velocity and pressure fields. One can simulate the entire two-dimensional flow by computing the distribution of vortices along the one-dimensional fluid-fluid interface. Hence vortex methods reduce the dimensionality of the problem by one.

The equations which underlie the vortex sheet method can be derived in the manner of Tryggvason and Aref (1983) and Meiburg and Homsy (1988a). One starts with the momentum and volume balance expressions $(2.1 \mathrm{a}, \mathrm{b})$. The two immiscible fluids, labeled 1 and 2 in Figure 4.1, are separated by a curved interface with unit tangent $\mathrm{s}$. The normal component of the fluid velocity is continuous across the interface and is equal to that of the interface. The tangential component is discontinuous across the interface, as shown in the figure. This causes the appearance of a vortex sheet. The strength $\gamma$ of this sheet is defined as

$$
\gamma=\left(\mathbf{u}_{2}-\mathbf{u}_{1}\right) \cdot \mathbf{s}
$$

where $\mathbf{u}_{1}, \mathbf{u}_{2}$ are the two fluid velocities at the interface. Multiplying the momentum equation (2.1b) for each fluid by $\rho g$, subtracting that corresponding to fluid 2 from that of fluid 1 , and taking dot product with $\mathrm{s}$ gives

$$
k\left(\nabla p_{1}-\nabla p_{2}\right) \cdot \mathbf{s}+k g\left(\rho_{1}-\rho_{2}\right) \mathbf{j} \cdot \mathbf{s}=\left(\mu_{2} \mathbf{u}_{2}-\mu_{1} \mathbf{u}_{1}\right) \cdot \mathbf{s}
$$

where $k=b^{2} / 12$ and $\mathbf{j}=\nabla z$. This can be coupled with (4.1) to yield 


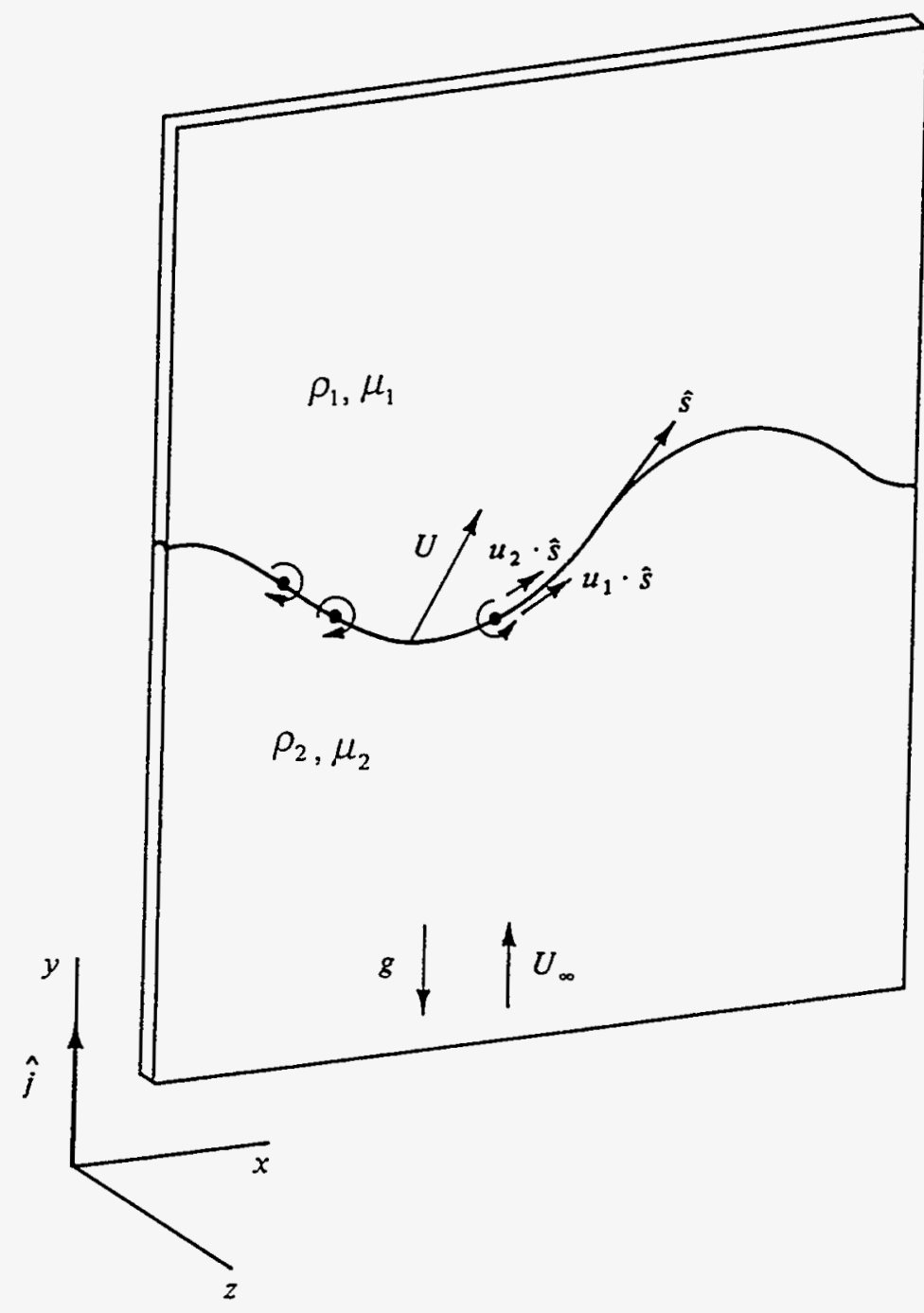

Figure 4.1. Sketch defining notation for vortex equations (after Tryggvason and Aref, 1983). 


$$
\gamma=\frac{\Delta \mu}{\mu_{m}} \mathrm{U} \cdot \mathbf{s}+\frac{k g}{\mu_{m}} \Delta \rho \mathbf{j} \cdot \mathbf{s}+\frac{k}{\mu_{m}} \nabla(\Delta p) \cdot \mathbf{s}
$$

where $\Delta p=p_{1}-p_{2}, \Delta \rho=\rho_{1}-\rho_{2}, \mu=\mu_{1}-\mu_{2}, \mu_{m}=\left(\mu_{1}+\mu_{2}\right) / 2$, and $\mathbf{U}=\left(\mathbf{u}_{1}+\mathbf{u}_{2}\right) / 2$. The term $\Delta p$ is related to the Laplacian of $\eta$, the perturbed coordinate of the front, by means of Laplace's formula (3.17). The velocity $U$ can be decomposed into the sum of $\mathbf{U}_{\mathrm{vor}}$, the velocity induced by the vortex sheet, and $\mathbf{U}_{\mathrm{pot}}$, the irrotational uniform velocity far from the front. $U_{\mathrm{vor}}$ is related to $\gamma$ via Birkhoff's (1954) integral formula

$$
\mathbf{U}_{\text {vor }}(s, t)=\frac{1}{2 \pi} \int \frac{\mathbf{z} \times\left[\mathbf{x}(s, t)-\mathbf{x}\left(s^{\prime}, t\right)\right]}{\left|\mathbf{x}(s, t)-\mathbf{x}\left(s^{\prime}, t\right)\right|^{2}} \gamma\left(s^{\prime}, t\right) d s^{\prime}
$$

where $\mathbf{z}$ is a unit vector normal to the plane of the Hele-Shaw cell, and $s$ is the distance of any point $\mathbf{x}(s, t)$ along the interface from some arbitrary point $\mathbf{x}\left(s_{o}, t\right)$ on the curve.

Numerical simulations typically involve representation of the vortex sheet by a row of marker points, leading to the so-called point vortex method. Knowing the position and velocity of each marker point at the beginning of a time step, $\Delta t$, each point is advanced to a new position a distance $\mathbf{U} \Delta t$ downstream. Together the new marker positions define a new interface at the end of $\Delta t$. The velocity and vorticity of each point at the new position are determined iteratively in the following manner. Based on the curvature of the interface, $\Delta p$ at each marker point is computed according to (3.17). Next, $\gamma$ is estimated according to (4.3) using velocities from the previous time step. A new velocity for the current time step is then computed by means of (4.4). This velocity is used to recompute $\gamma$ again, which in turn yields a new velocity, and so on. The iterative process continues until adjustments are no longer deemed necessary for both velocity and vorticity. The calculation then proceeds to the next time step.

At each iteration, (4.4) requires $N$ terms to be computed for each of $N$ marker points defining the front. To maintain accuracy for a highly distorted interface, $N$ may have to become very large and the computational burden may become excessive. It may be reduced by means of vortex-in-cell (VIC) methods which couple the Lagrangian interface with an Eulerian grid. The VIC idea has been introduced by Christiansen (1973). Instead of (4.4), the vorticity and velocity fields are related via the Poisson equation

$$
\nabla^{2} \Phi=-\omega
$$


where $\omega=\partial u_{y} / \partial x-\partial u_{x} / \partial y$ is vorticity and $\Phi$ is stream function defined such that $\nabla \Phi=\left(-u_{y}, u_{x}\right)^{\mathrm{T}}, u_{x}$ and $u_{y}$ being the velocity components along the $x$ and $y$ coordinates, respectively. The vorticity $\omega$ is related to $\gamma$ through

$$
\omega(x, t)=\sum \gamma\left(s_{i}\right) \Delta s_{i} \delta\left[\mathbf{x}-\mathbf{x}_{i}(t)\right]
$$

where $s_{i}$ and $\mathrm{x}_{i}$ correspond to the $i$-th elemental vortex, $\Delta s_{i}$ is the corresponding arc length, and $\delta$ is the Dirac delta function. In general, it is most convenient to solve the Poisson equation on a fixed (Eulerian) grid. Since spatial resolution is usually not much higher than the grid spacing, it does not help to have more than about one vortex per grid cell. A better way to maintain accuracy is to rely on local grid refinement when and where the interface is distorted.

As the vortex sheet distorts due to fingering, some vortices move far apart and some tend to cluster. Where the vortices are far apart, interpolation renders the interface smoother and hence more stable than it might be otherwise (Birkhoff, 1954). It is therefore advisable to redistribute the vortices evenly along the interface, and add new ones, periodically during the computation.

Figure 4.2 illustrates some typical finger patterns simulated by Tryggvason and Aref (1983) using VIC with various mobility ratios on a grid of $256 \times 256$ cells. The initial condition at the interface was the same for each run and consisted of an arbitrary collection of waves having small amplitudes and diverse wavelengths. The figure shows that there is little visible difference between early finger patterns corresponding to different mobility ratios, $A$. However, the mobility ratio clearly affects finger patterns at later time. When the two fluids have identical viscosities $(A=0)$, all incipient fingers grow without any discernible shielding of some by others. With time, the longest fingers develop into narrow necks with heads at their tips (breakage of these necks is disallowed in the numerical code). As the viscosity contrast between the fluids increases, shielding becomes more and more pronounced. The heads of dominant fingers elongate and widen as $A$ grows toward 1 . The fingers elongate linearly with time except at very early time. It increases with mobility ratio and mean displacement velocity but remains independent of modified capillary number.

In a subsequent paper, Tryggvason and Aref (1985) simulated finger development from a single initial sine wave of small amplitude, and from a superposition of this and another wave having twice the wavelength and one fifth the amplitude. Some results are 


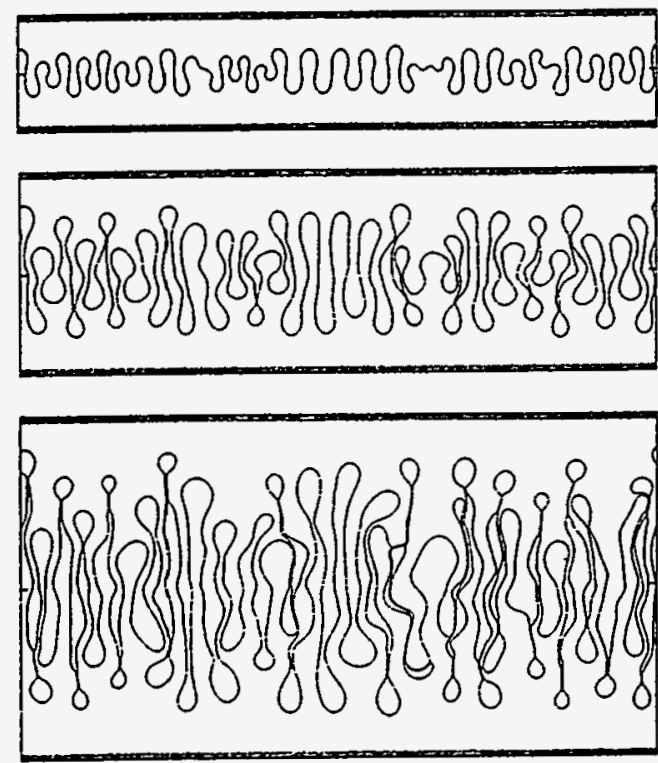

(a)
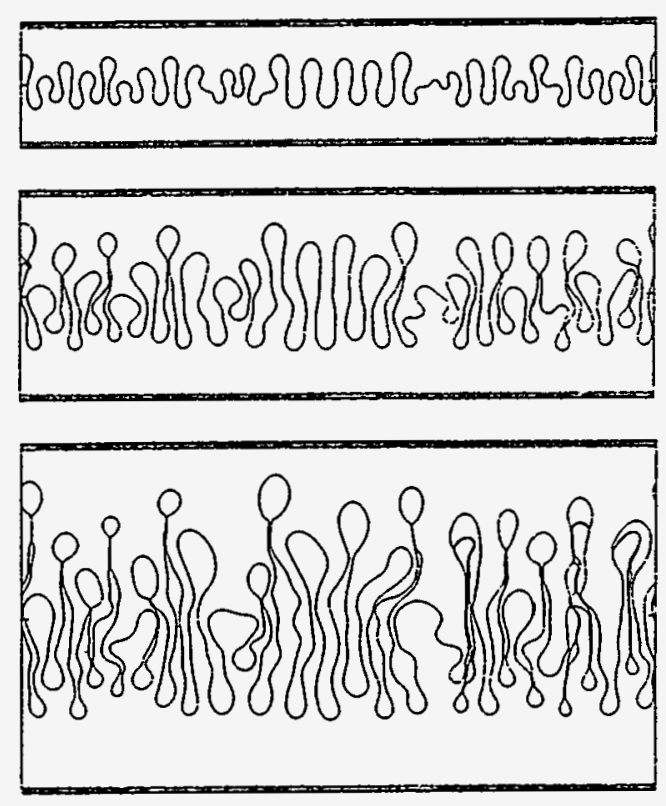

(b)

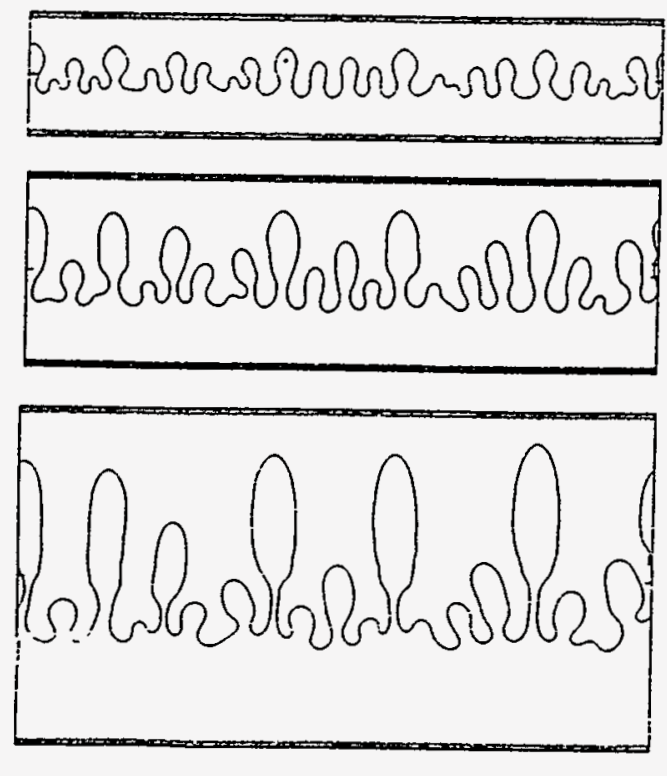

(c)

Figure 4.2. Stages of interface evolution using vortex-in-cell method with (a) $A=0$, (b) 0.5, (c)1 (after Tryggvason and Aref, 1983). 
illustrated in Figure 4.3. Their main finding was that whereas a single wave results in a more-or-less uniform array of like fingers which show little dependence on $A$, superimposed waves result in finger patterns similar to those obtained from random perturbations.

Meiburg and Homsy (1988b) used the point vortex method to simulate finger development at modified capillary numbers $C a^{\prime}=530$ and 833 with $A=1$ from a single initial sine wave of small amplitude. At $C a^{\prime}=1111$ and 2500, small random perturbations were superimposed on the velocities of the marker points at each time step. The results, illustrated in Figure 4.4, suggest that fingers evolving from a single sine wave at lower $C a^{\prime}$ values are smooth and grow asymptotically at a linear rate. Fingers evolving from random perturbations at higher $C a^{\prime}$ values also grow asymptotically at a near linear rate. However, they exhibit spreading, shielding and splitting of their tips in the process.

\subsection{Random Walk Models}

Random walk models are based on the fact that the probability, $P(\mathbf{x}, \mathrm{t})$, that a random walk originating far from a point $\mathbf{x}$ will lead to this point at time $t$ obeys the Laplace equation. It was used by Witten and Sender $(1981,1983)$ to model diffusionlimited aggregation (DLA) of small particles. In their model, a steady stream of particles is released far from a cluster of similar particles, each performing a random walk toward the cluster. When a particle strikes the cluster, it sticks to it and thereby contributes to its chaotic growth. This results in typical dendritic patterns of the kind illustrated in Figure 4.5. The model is described mathematically by (Witten and Sender, 1983)

$$
\nabla^{2} P(\mathbf{x}, t)=0
$$

subject to a uniform release rate at infinity,

$$
U=\left.\chi \nabla P\right|_{\infty}
$$

where $\chi$ is diffusion coefficient. As particles have zero probability of penetrating a cluster or remaining on its surface (due to eventual coverage by later arrivals), $P$ must satisfy

$$
P=0
$$

on the boundary of the aggregate. The aggregate then grows, its boundary expanding at a 


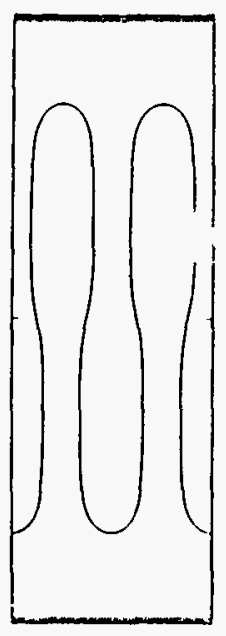

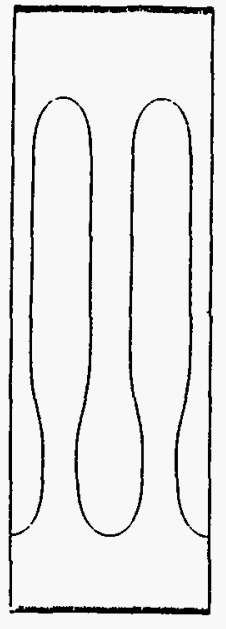

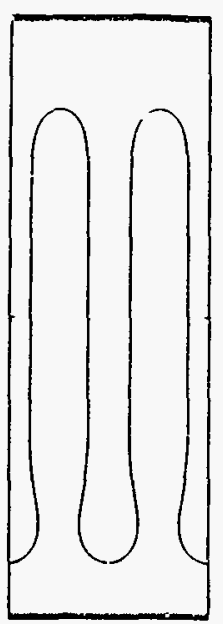

(a)
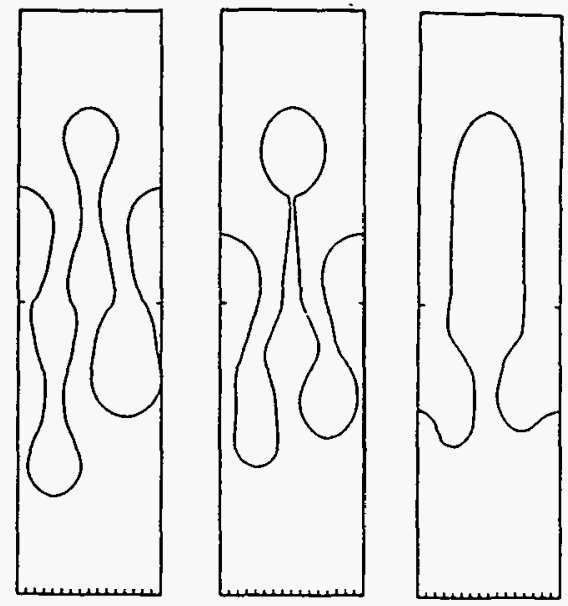

(b)

Figure 4.3. VIC prediction of finger development from (a) a single sine wave and (b) two superimposed sine waves. In each case $A=0$ at left, $A=0.5$ at center, $A=1.0$ at right (after Tryggvason and Aref, 1985).

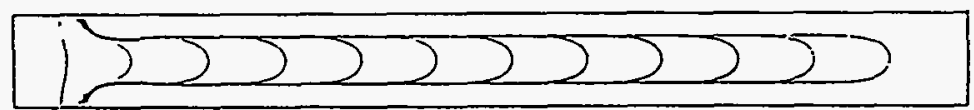

(a)
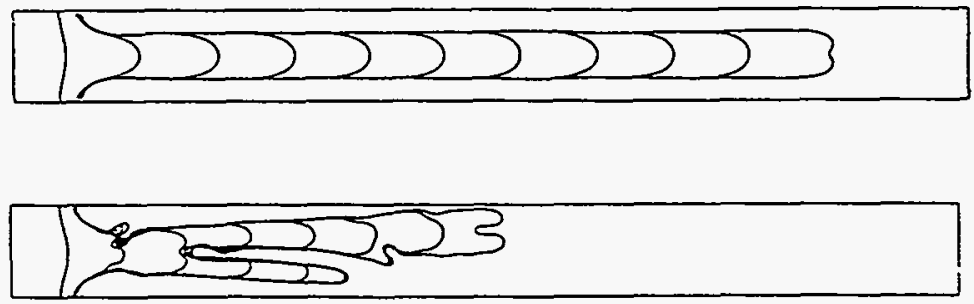

(c)

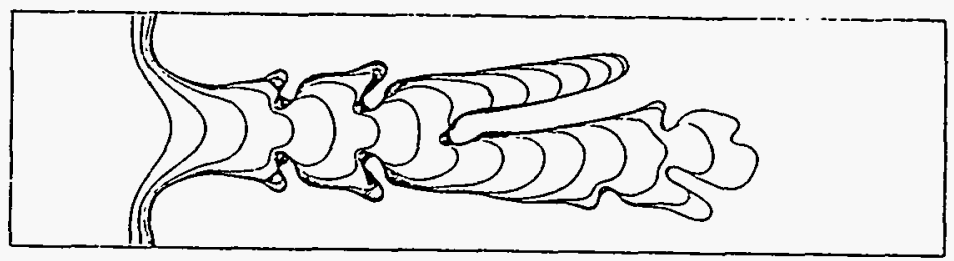

(d)

Figure 4.4. Point vortex results with $A=1$ and (a) $C a^{\prime}=530$; (b) $C a^{\prime}=833$; (c) $C a^{\prime}=1111$; (d) $C a^{\prime}=2500$ (after Meiburg and Homsy, 1988b). 


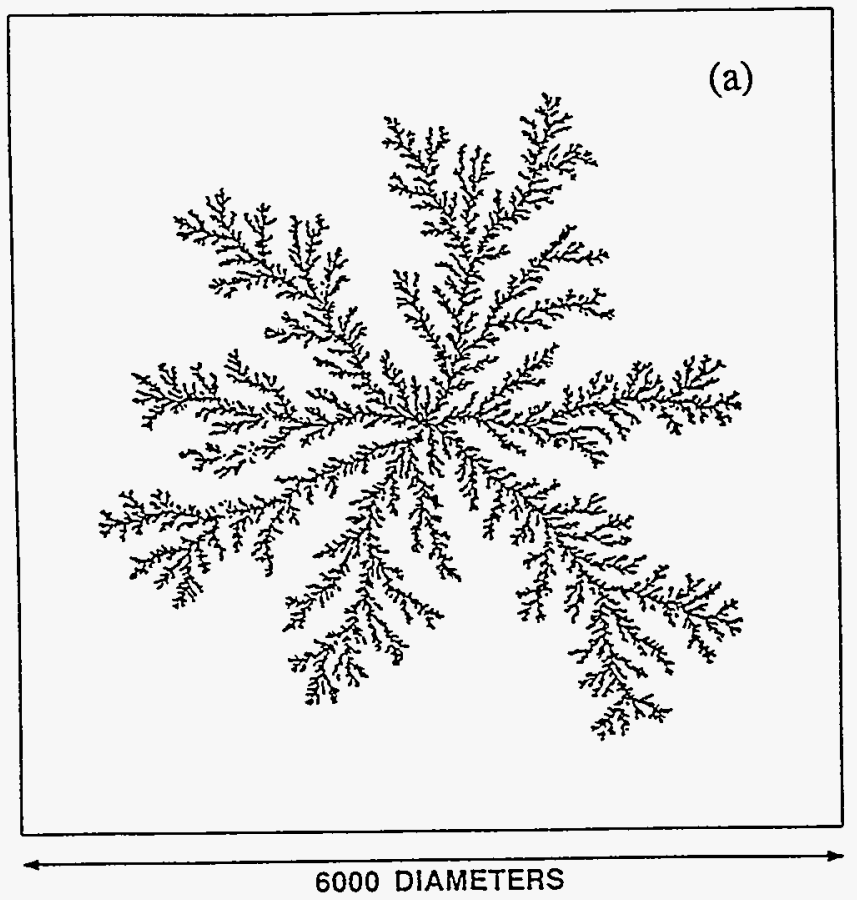

(b)

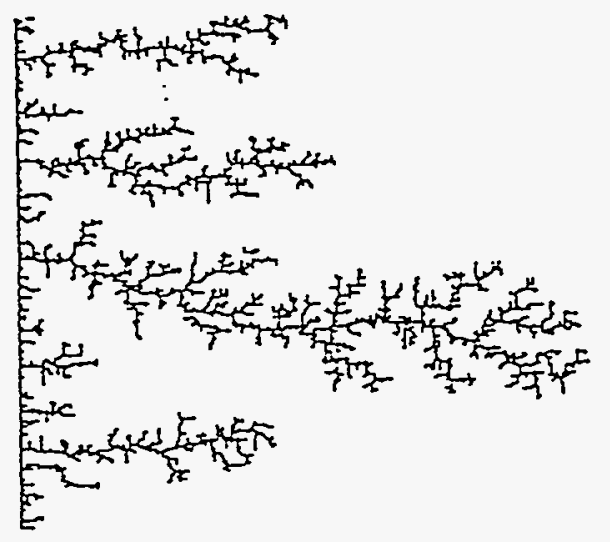

Figure 4.5. Fractal structures produced by DLA: (a) With a particle seed (after Meakin, 1991) and (b) with a line seed (after Lenormand et al., 1988). 
rate (Bensimon et al., 1986)

$$
v_{n}=\chi(\nabla P)_{n}
$$

where the subscript $n$ implies normal direction. If particles are reflected from the side walls of the container, the normal probability flux there is zero,

$$
(\nabla P)_{n}=0 .
$$

Paterson (1984) and Tang (1985) have pointed out an analogy between the above DLA model and the motion of a fluid-fluid interface in a Hell-Shaw cell or a porous medium when gravity, interfacial tension and the viscosity of one fluid are zero. In the inviscid fluid, the pressure $p$ is constant and may be set to zero. In the viscous fluid, the pressure satisfies the Laplace equation

$$
\nabla^{2} p=0
$$

subject to a uniform flux at infinity,

$$
U=-\left.\frac{k}{\mu} \nabla p\right|_{\infty}
$$

At the interface $p=0$ and along impermeable walls

$$
(\nabla p)_{n}=0 .
$$

The interface moves at the Darcian rate

$$
v_{n}=-\frac{k}{\mu}(\nabla p)_{n} .
$$

The analogy with (4.7) allows simulating the interface by means of a random walk model. One result of such a simulation has been shown in Figure $4.5 \mathrm{~b}$. It illustrates that, due to the inviscid nature of one fluid and the absence of surface tension, the interface is so unstable as to produce a fractal structure.

Kadanoff (1985) and Liang (1986) modified the DLA method to include surface tension. The phenomenon was mimicked by allowing particles to be emitted from the interface with probability proportional to its curvature and ended at possibly another point on the interface. As particles change their positions on the interface, its curvature 
changes. Liang (1986) tested this idea by simulating the evolution of an oddly shaped two-dimensional air bubble in water. In this simulation, particles were allowed to move only from interface to interface through water, not from outside to the interface. The bubble evolved toward its theoretical equilibrium state, a circle.

Random walk simulations are usually done on a square lattice. The domain is subdivided into a grid of occupied and unoccupied cells representing displacing and displaced fluids (e.g., air and water) as shown in Figure 4.6. To simulate a uniform fluid velocity far from the interface, particles are released at a uniform rate from a distant line or area source and walk randomly through the unoccupied region. Their walk terminates at the interface where they form a growing cluster. Random particle movement from interface to interface simulates surface tension. When surface tension is pronounced, this may become computationally demanding. When it is weak, random walk models tend to produce highly irregular interfaces. Tang (1985) and Liang (1986) considered such irregularities to be inconsistent with the deterministic nature of the phenomenon. To obtain smoother surfaces, they proposed avoiding any modifications to the interface until a sufficiently large number of particles have reached it or moved along it. The optimum number of such particles has remained unspecified.

Some results of simulations by Liang (1986) are illustrated in Figure 4.7. In the figure, $\mathrm{Ca}^{\prime}$ increases and surface tension decreases from left to right. Correspondingly, we observe a smooth finger on the left, finger splitting at the center, and a ramified pattern on the right. A similar trend has been observed experimentally by Maxworthy (1987) and Tabeling et al. (1987).

Random walk models are useful for highly unstable flows which are difficult to treat by other methods. However, as pointed out by Kadanoff (1985), they are not well suited for nonuniform media. To date, neither gravity nor finite viscosity ratios have been incorporated in random walk models.

\subsection{Pore-Scale Percolation Models}

The details of fluid movement in porous media are difficult to analyze due to the complex geometry and topology of the pore space. One recent trend has been to view the porous medium as a network of spherical pores connected by narrower cylindrical throats (Lin and Cohen, 1982; Dullien, 1992). The picture is often idealized further by considering a regular lattice (Figure 4.8) in which the nodes represent pores and the grid lines represent throats. In percolation theory, the nodes are called sites and the grid lines 


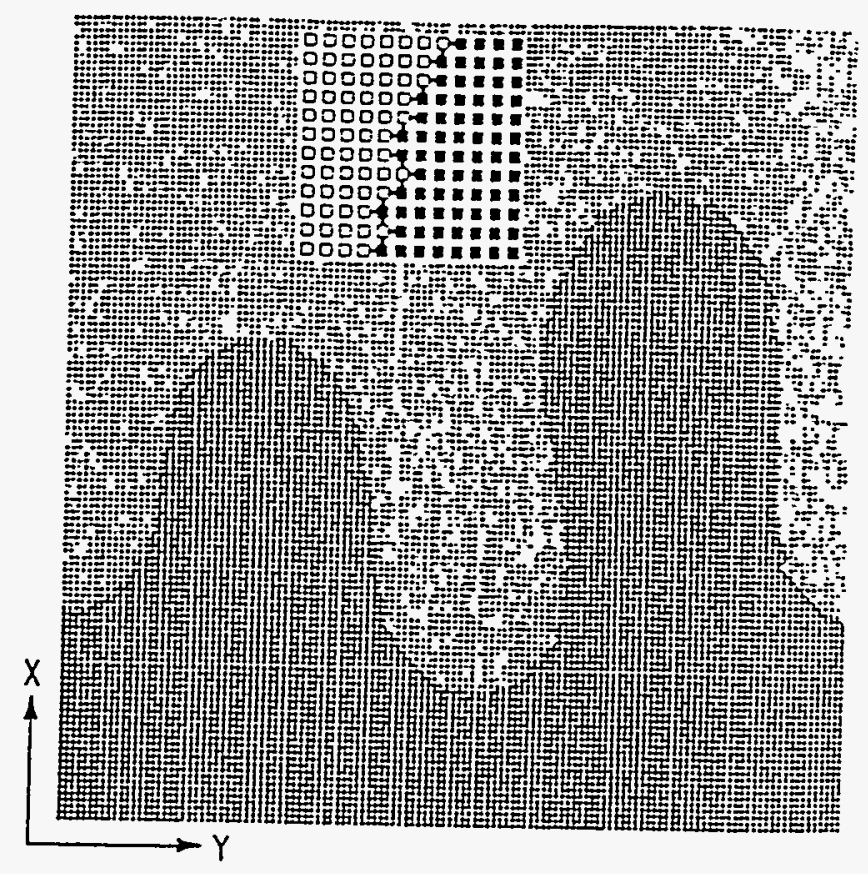

Figure 4.6. Square lattice for random walk simulation. Dark region represents air, light region water (after Liang, 1986)
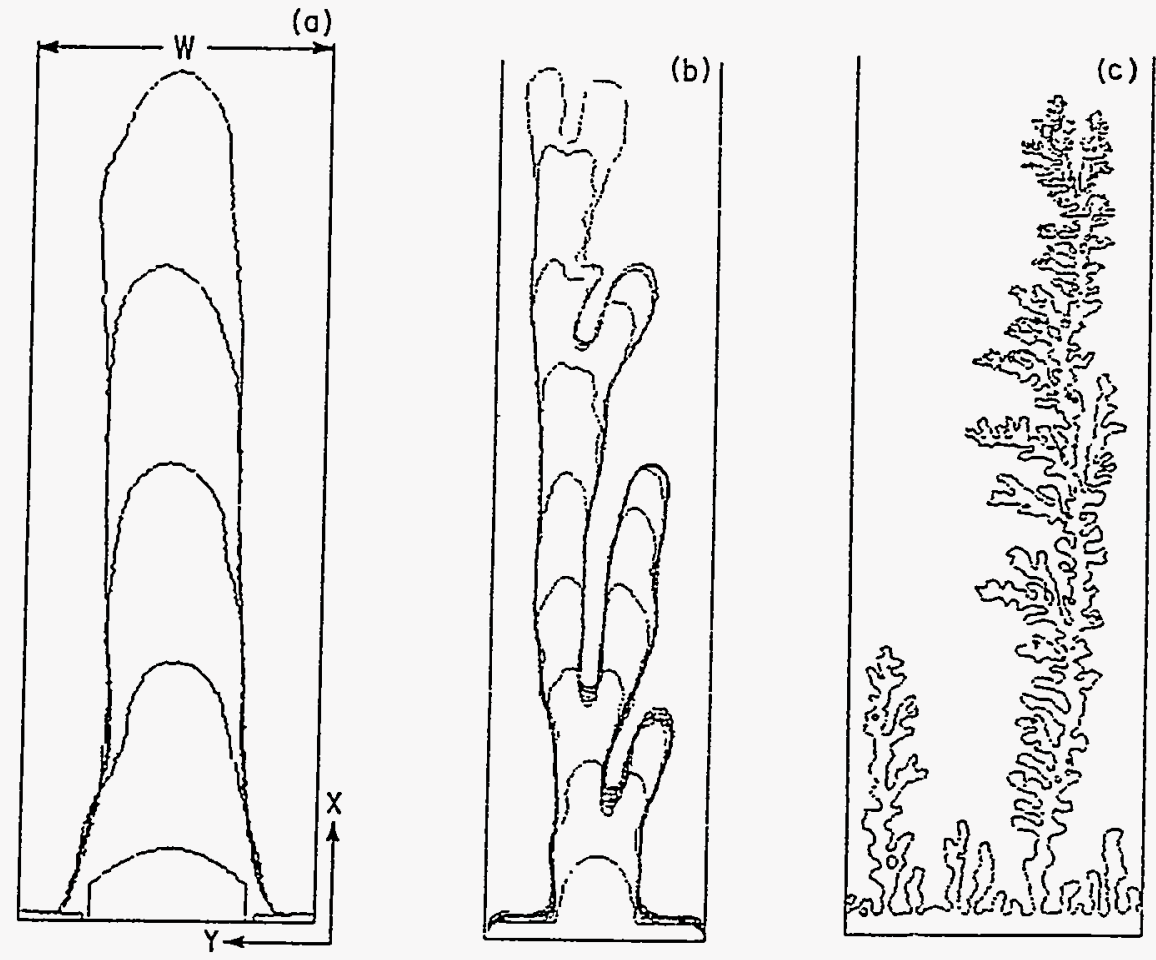

Figure 4.7. Random walk simulations with (a) $C a^{\prime}=555$ and $N=20$, at the onset of instability; (b) $C a^{\prime}=2.5 \times 10^{3}$ and $N=15$, at the beginning of tip splitting; (c) $C a^{\prime}=2 \times 10^{5}$ and $N=3$, finger evolves into a ramified structure (after Liang, 1986). 
are termed bonds. Lattice patterns, the radii of sites and bonds, and bond lengths may be made to mimic pore space geometry by selecting some of them at random from probability distributions inferred on the basis of observed pore structures (Ferrand et al., 1990). Each bond and site are typically assigned a threshold capillary pressure

$$
P_{c}=\frac{T}{R}
$$

where $R$ is radius and $T$ is surface tension. A non wetting fluid cannot enter a bond or a site until the capillary pressure builds up to equal or exceed the threshold pressure. Clearly, bonds and sites having larger radii tend to be invaded by a nonwetting fluid before those having smaller radii. The converse is true for invasion by a wetting fluid. Since throats (bonds) are generally narrower than pores (sites), the former tend to control drainage and the latter imbibition in media containing air and water. These idealized porescale models are sometimes termed percolation models even though they do not always conform to standard percolation theory. An excellent recent reference on pore-scale models and percolation theory as applied to single-phase flow and transport is the book by Adler (1992).

Lenormand et al. (1988) simulated horizontal displacement of a wetting fluid of viscosity $\mu_{1}$ and density $\rho_{1}$ by a non-wetting fluid of viscosity $\mu_{2}$ and density $\rho_{2}$ in a network of capillary pores and throats. They assumed that pressure drops occur only in the throats. A system of algebraic mass and momentum equations was written for fluid pressures at the sites, subject to prescribed initial as well as boundary conditions. The flux in each bond was made proportional to the difference between pressures at its two ends (sites) according to the Poiseuille equation. Each bond was associated with a threshold pressure, rendering the flow equations nonlinear and requiring that they be solved iteratively. The interface between the two fluids was advanced based on the calculated flow rates. Time steps were chosen so as to completely fill one pore. This means that the interface was moved in all the pores until it reached one of the throats. Some of their results for various viscosity ratios $M=\mu_{1} / \mu_{2}$ and capillary numbers $C a^{\prime}$, defined here as $U \mu_{2} / A T$ where $A$ is cross sectional area, are shown in Figure 4.9. Figure 4.9a shows how percolation patterns vary with capillary number at the high (unfavorable) ratio of $M=2 \times 10^{5}$. The pattern corresponding to the highest $C a^{\prime}$ value exhibits a ramified structure reminiscent of the DLA pattern. The authors attributed it predominantly to viscous forces and considered it to be characteristic of viscous fingering. Patterns corresponding to smaller $C a^{\prime}$ values were attributed by them primarily to capillary forces. 


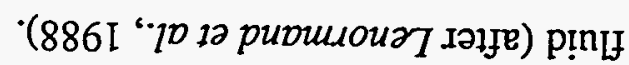

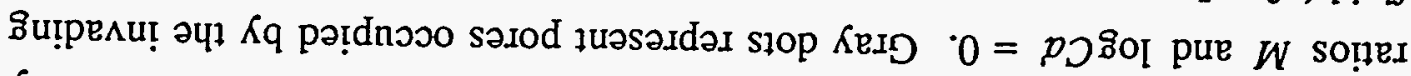

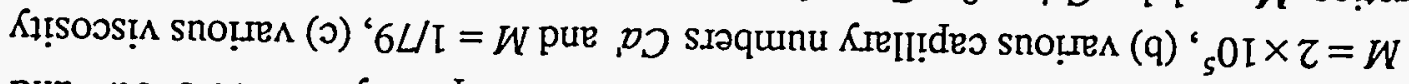

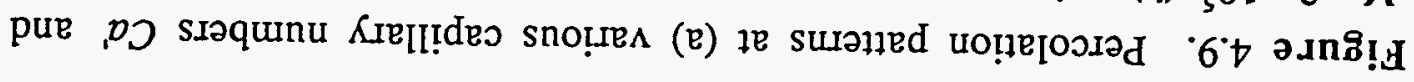
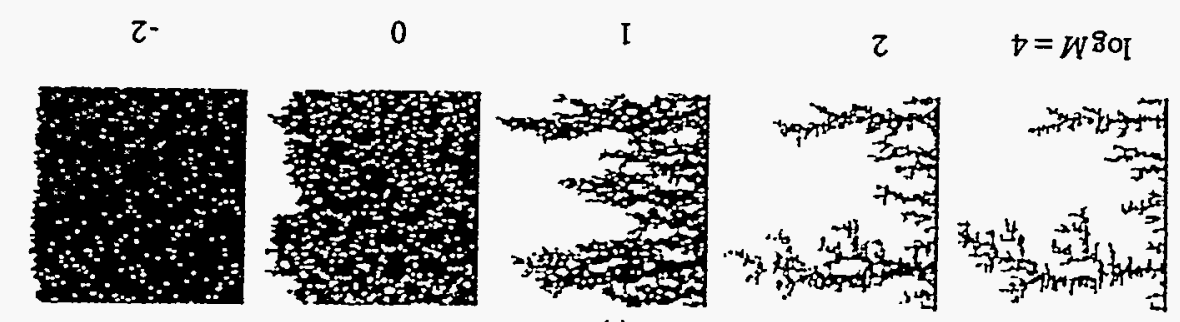

(ग)

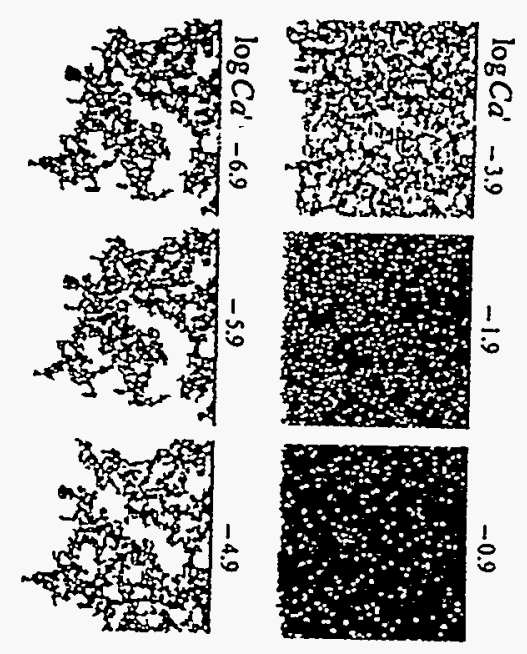

(q)
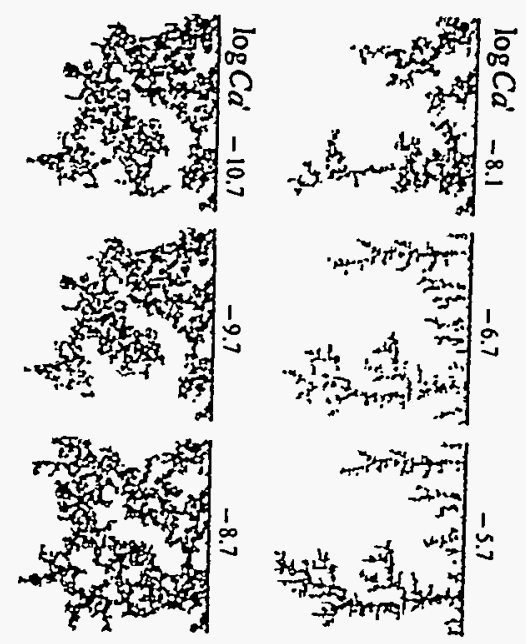

(v)

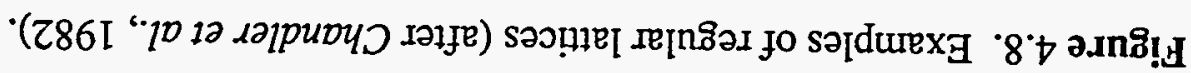
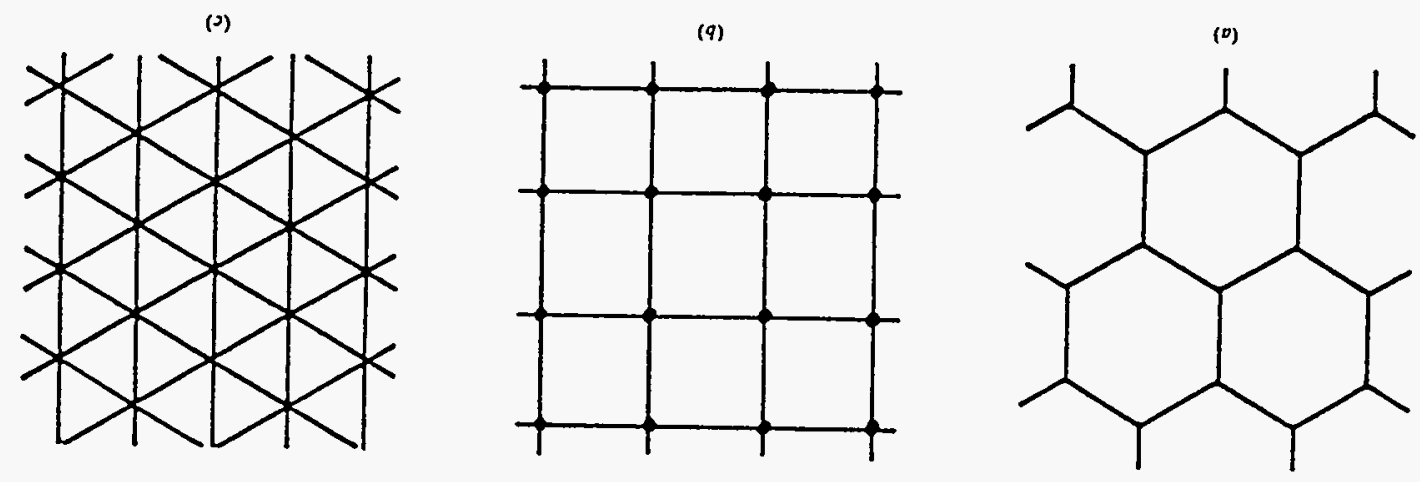
Contrary to the viscous pattern, the capillary patterns include branches which grow sideways, backward, and may completely loop around the invaded fluid. Figure 4.9b shows percolation patterns at the low (favorable) viscosity ratio of $1 / 79$. A continuous transition is evident between stable displacement at high $\mathrm{Ca}^{\prime}$ values and unstable displacement at low $\mathrm{Ca}^{\prime}$ values. Note the similarity between the unstable patterns corresponding to minimum $\mathrm{Ca}^{\prime}$ values in Figure 4.9a and 4.9b. Figure 4.9c shows how changes in $M$ affect the percolation pattern when $\log C a^{\prime}=0$. The percolation is seen to be stable when $M$ is favorable $(M<1)$ and unstable when it is unfavorable $(M>1)$.

According to the stability analysis in Chapter 2, displacement of a less viscous fluid by a more viscous fluid in a uniform medium, in the absence of gravity, should yield an unconditionally stable interface as both viscous and capillary forces act to stabilize it. Hence the unstable percolation patterns in Figure $4.9 \mathrm{~b}$ appear to result from medium heterogeneity, or preferential flow, on the pore scale. The reference to capillary fingering by Lenormand et al. (1988) might thus be a misnomer.

Under very slow flow, viscous forces are vanishingly small and percolation depends solely on gravity and local capillary forces at the interface. This case is amenable to analysis by a technique known as invasion percolation which is computationally much more efficient than the standard approach though it has traditionally disregarded gravity (Lenormand and Bories, 1980; Chandler et al, 1982; Wilkinson and Willemsen, 1983; Wilkinson, 1986). In this method, each site and bond at the interface is ranked according to its level of participation in movement: During invasion of a nonwetting fluid, the highest rank is associated with the highest threshold pressure and, during invasion of a wetting fluid, with the lowest threshold pressure. This is so because the former occurs first where the threshold pressure is highest, and the latter occurs first where this pressure is lowest. During any time step, only the highest ranking bonds and sites participate in motion. A new ranking is made and the interface is allowed to advance again. The method is computationally efficient because there is no need to solve a system of coupled nonlinear equations. It is however unsuitable for faster motions where viscosity becomes important. A percolation pattern computed by this technique is shown in Figure 4.10. It closely resembles the pattern corresponding to $\log C a^{\prime}=-10.7$ in Figure 4.9a.

Glass (1993) modified the invasion percolation technique to include gravity and macroscopic interfacial curvature during water infiltration through a rough-walled fracture. In his model, the fracture is represented by a square grid of cells, each having a 


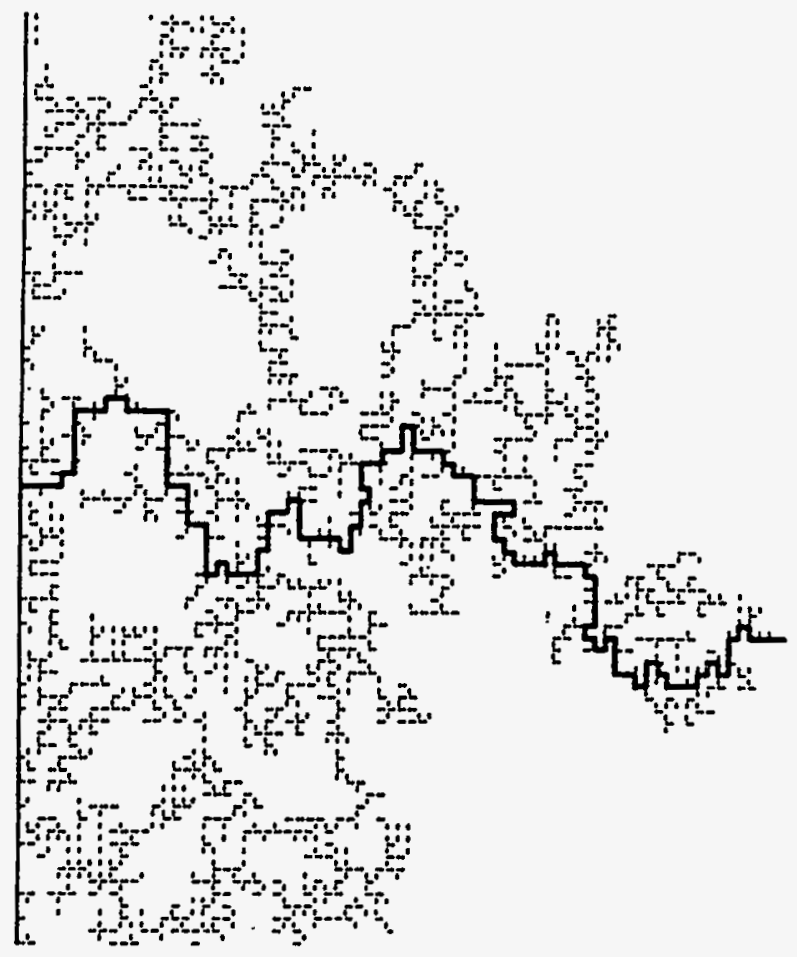

Figure 4.10. Invasion percolation pattern. Heavy line shows continuous path between entrance and exit (after Lenormand et al., 1988). 
randomly assigned uniform aperture. The author ranked all cells connected to the interface in terms of their total aperture filling potentials, defined as

$$
h=-\frac{T}{\rho g}\left(\frac{1}{r_{1}}+\frac{1}{r_{2}}\right)+z \cos \delta
$$

where $z$ is a coordinate coinciding with the dip of the fracture, $\delta$ is the angle between $z$ and the vertical (a vector pointing in a direction opposite to that of gravity), $r_{1}$ is principal radius of interface curvature normal to the plane of the fracture (given by half the local aperture), and $r_{2}$ is macroscopic principal radius of interface curvature parallel to the same plane. The first term on the right represents capillary pressure potential and the second gravity potential. During any time step, only one cell with the most negative filling potential is allowed to be invaded. This modifies the set of cells in contact with the interface and the in-plane curvature of the interface. The filling potentials are updated to reflect this and a new invasion step is initiated. Figure 4.11 illustrates the invasion of water into an air-dry rough fracture at $\delta=180^{\circ}$ (vertical infiltration), $138^{\circ}, 120^{\circ}, 104^{\circ}$, $90^{\circ}$ (horizontal infiltration), and $0^{\circ}$ (capillary rise). When in-plane curvature is not considered (Figure 4.11a), fingers are controlled by microscopic variations in aperture. When in-plane curvature is considered (Figure 4.11b), macroscopic fingers develop which more closely resemble experimental observations. As the effect of gravity decreases when $\delta$ diminishes from $180^{\circ}$ to $90^{\circ}$, the percolation pattern in Figure 4.11 a becomes more intricate and the invaded area increases. Under horizontal invasion at $\delta=90^{\circ}$, branching takes place in all directions as was seen earlier in Figures 4.9 and 4.10. During capillary rise at $\delta=0^{\circ}$, the flow is stable on the average due to gravity.

\subsection{Conventional Numerical Methods}

Conventional numerical methods can be used to simulate fingering by solving the standard macroscopic equations of immiscible two-phase flow in porous media on a grid. Very fine grids may be required to resolve narrow fingers and to avoid numerical smearing (dispersion) and instabilities. Some standard finite difference and finite element numerical schemes are prone to artificial smearing and/or instability unless special precautions are taken to prevent them. Various nonstandard approaches have been proposed to help eliminate, or minimize, these numerical artifacts. Among them, Eulerian-Lagrangian techniques appear to be quite promising. Originally, these techniques have been developed for miscible advective-dispersive transport (e.g., Neuman, 

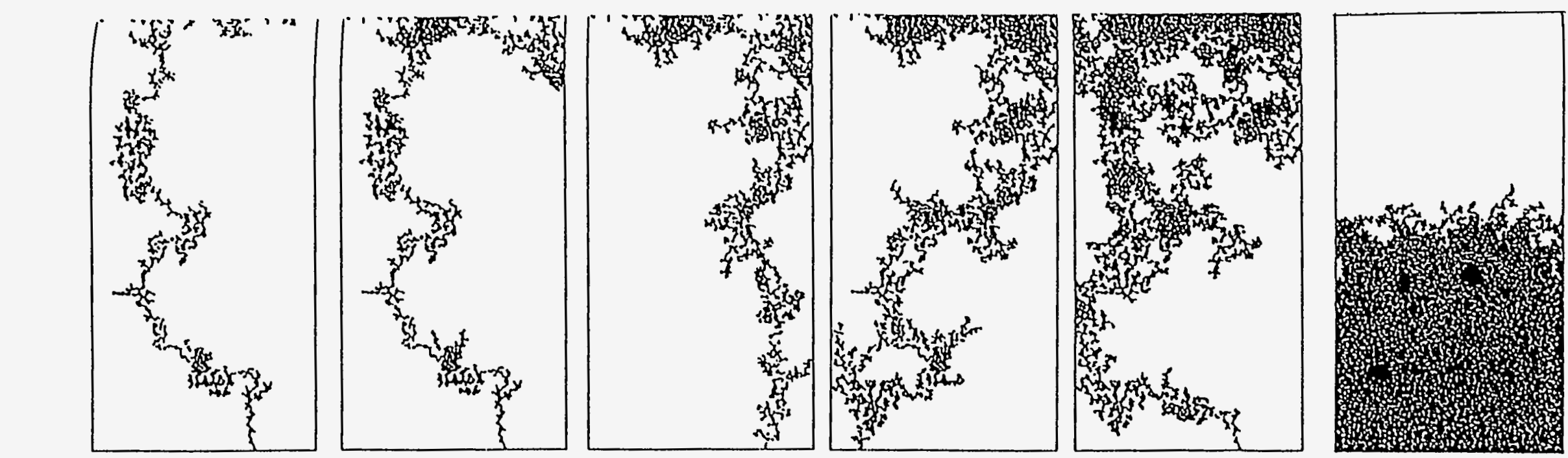

(a)
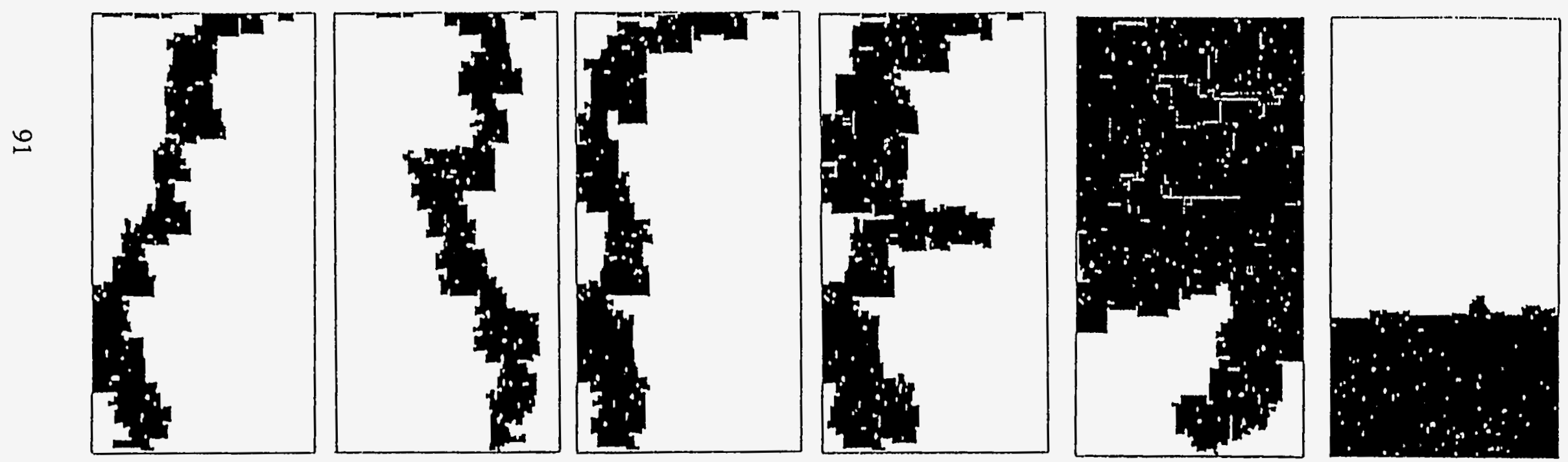

(b)

Figure 4.11. Invasion percolation of water into an air-dry rough-walled fracture: (a) in-plane curvature not considered, (b) in-plane curvature considered. Gray regions denote water and light regions air. From left to right $\delta=180^{\circ}, 138^{\circ}, 120^{\circ}$, $104^{\circ}, 90^{\circ}$, and $0^{\circ}$ (after Glass, 1993). 
1981; 1984; Douglas and Russell, 1982; Neuman and Sorek, 1982; Ewing et al., 1984; Baptista, 1987; Celia et al., 1990; and Herrera et al., 1993) but should also be applicable to immiscible displacement (Douglas and Yirang, 1988; Ewing, 1991). The idea is to formally split the problem into an advective and a dispersive part, then solve the advection problem in a Lagrangian framework (typically by a method of characteristics) and the dispersion problem by an Eulerian finite difference or finite element scheme. A closely related alternative is the flux-corrected approach developed by Borie and Book (1973, 1976) and Zalesak (1978) for transport which has recently been used by Christie (1989) and Hill et al. (1994) to simulate immiscible displacement. This technique also relies on operator splitting but it solves the advective problem in an Eulerian framework by means of a low-order approximation and a higher-order correction. The low-order approximation produces excessive numerical diffusion to avoid artificial ripples. The higher-order correction counteracts this numerical diffusion. We will see later that the approach appears capable of simulating fingers during water infiltration into a soil.

To initiate fingers in a uniform medium, it may be necessary to perturb the flow field artificially. King et al. (1985) used a standard finite difference scheme to simulate two-phase immiscible displacement of oil by water in the absence of gravity (details on simulation domain and gridblock size were not given). To initiate instability, he perturbed the initial water saturation contours by means of a single parabolic kink. The amplitude of this kink was zero at full saturation and increased gradually with saturation to a small maximum value. Figure 4.12 shows the evolution of the kink with time. Each amplitude grew at a near constant rate without splitting or deforming the contours. The rate of growth increased with viscosity ratio and initial amplitude but decreased with capillary pressure.

Hughes and Murphy (1988) simulated immiscible displacement in a uniform medium by combining conventional and Monte Carlo approaches. Spatial and temporal variations in the saturation of the displacing fluid were computed by finite-differences. Local changes in saturation during a time step, normalized by the sum of changes over the entire grid, were interpreted to represent a point probability density function (pdf) as illustrated in Figure 4.13a. By selecting a random number between zero and one, a gridblock was identified from the corresponding cumulative distribution function (cdf) as shown in Figure 4.13b. The sum of the changes in saturation over the entire grid that had been calculated from finite differences was allocated to this gridblock. A time step was selected so as to insure that the assigned change in saturation is either equal to, or is some 
(a)
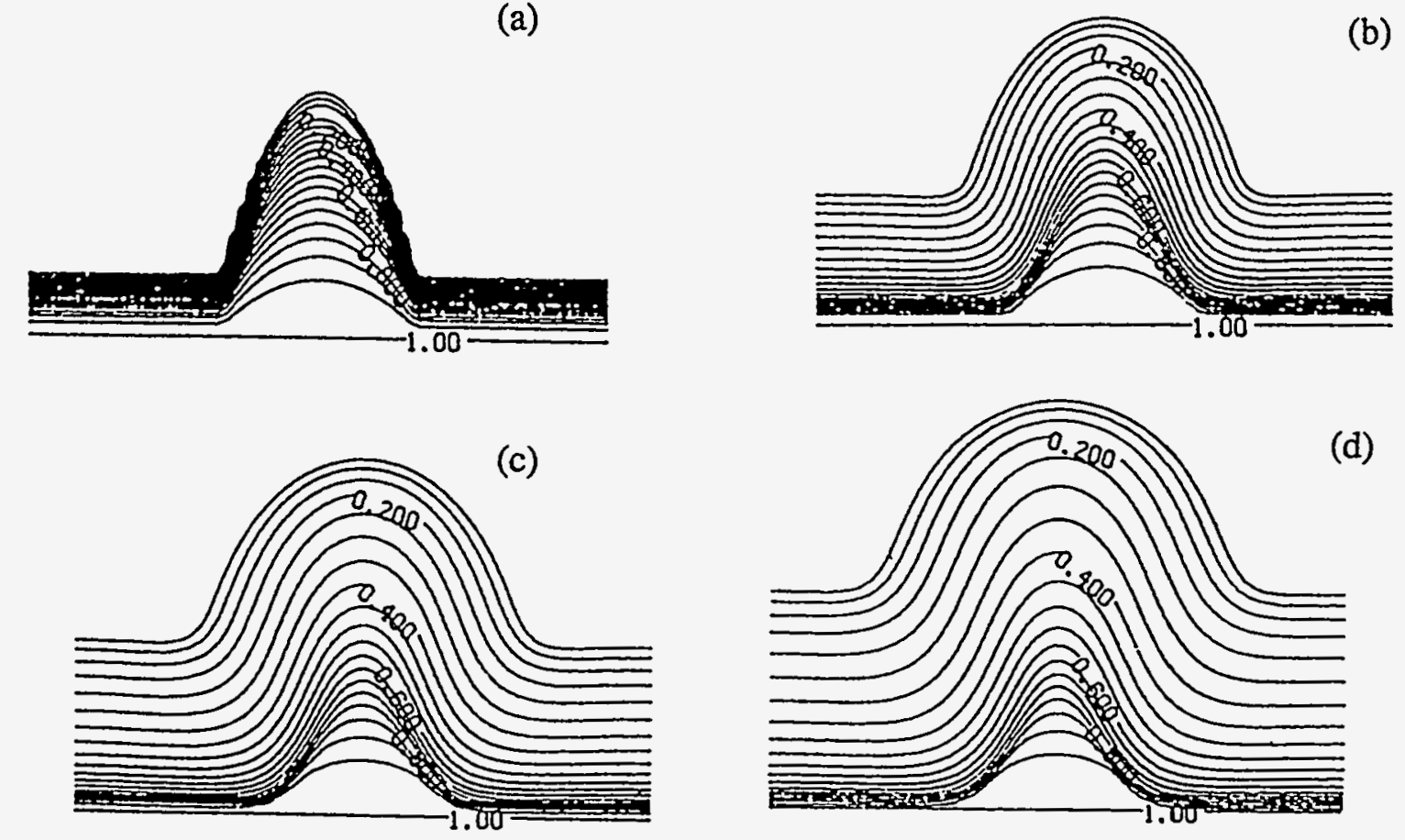

Figure 4.12. Contours of water saturation during displacement of oil at four equally spaced time steps. Capillary pressure is $0.02 \mathrm{~atm}$ and viscosity ratio is 10 (after King et al., 1985). 

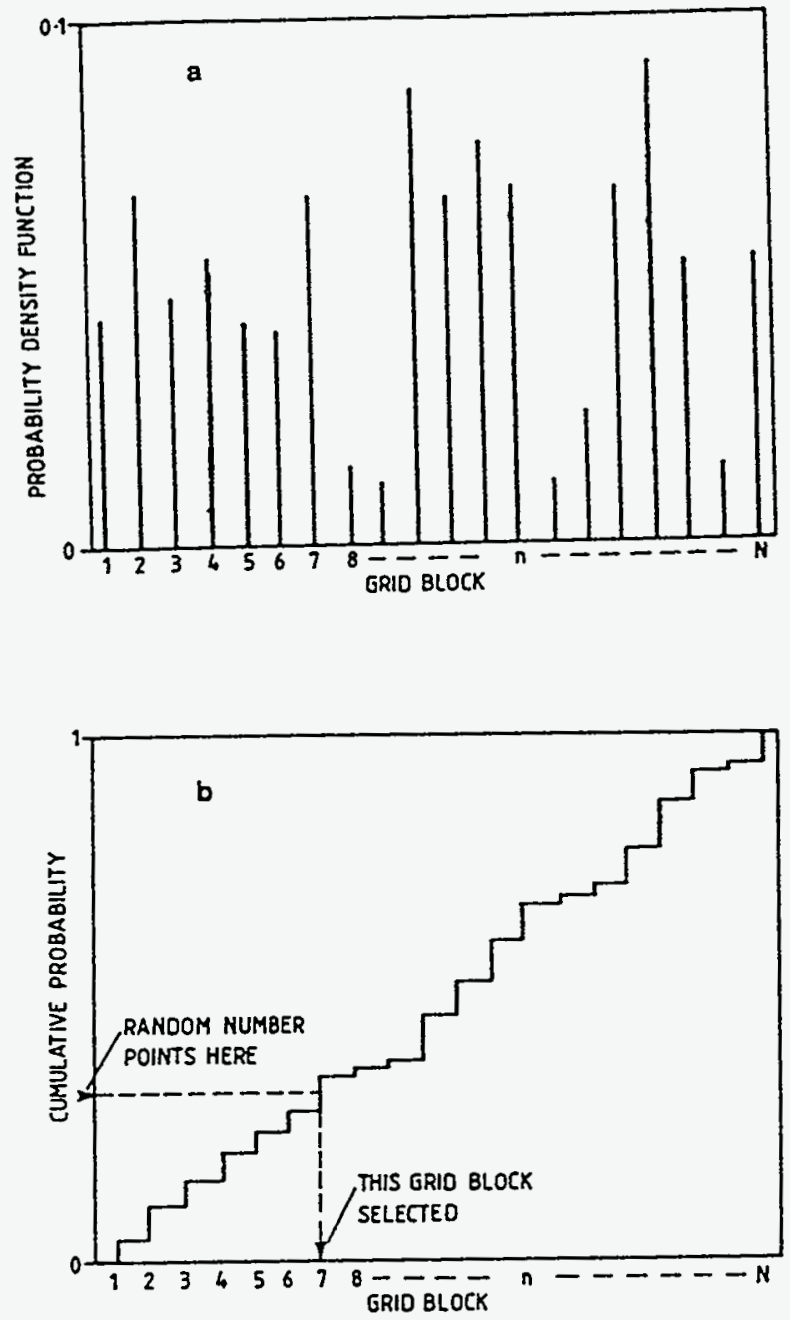

Figure 4.13. Interpretations of (a) change in saturation as a probability densit: function and (b) random selection of gridblock (after Hughes and Murphy, 1988). 
reciprocal integer fraction of, the maximum possible saturation change in the gridblock. A new change in saturation across the grid over a time step was then computed by finite differences, and so on. Figure 4.14 shows simulated water saturations at breakthrough during displacement of oil at $M=8$ and $M=99$ in the absence of gravity and capillarity. The grid contains $100 \times 50$ square cells of unspecified size. At $M=8$, the fraction of pore volume injected till breakthrough was 0.351 , and the invading water had fingered into $71.1 \%$ of the gridblocks. At the higher $M$ value of 99 , the fraction injected decreased to 0.107 and so did the invaded area down to $63.2 \%$. These results proved to be insensitive to the seed of the random number generator used by the authors, but details of the saturation profiles did vary from seed to seed.

Christie (1989) employed two approaches to trigger instabilities during the simulation of immiscible displacement by the flux-corrected method. In the first approach, a low-amplitude perturbation was superimposed on the interface at time $t=0$. In the second, permeability was made to vary randomly across the field with a log-normal distribution, small variance and no autocorrelation. Both approaches gave qualitatively similar results. Figure 4.15 shows results corresponding to horizontal oil displacement by water at $M=10$ and either moderate or tenfold lower capillary pressure on a grid of $205 \mathrm{x}$ 205 cells (of unspecified size). An increase in capillarity is seen to have a stabilizing effect by lowering the amplitude and frequency of fingers.

Blunt et al. (1994) used a $200 \times 100$ finite difference grid of unspecified cell size to simulate immiscible displacement of oil by gas at a viscosity ratio of 89 by disregarding gravity and capillarity. Fingers were triggered by a normally distributed random permeability field with a $5 \%$ variance and no autocorrelation. Figure 4.16 a depicts gas saturation contours after displacement of 0.1 pore volumes, and Figure $4.16 \mathrm{~b}$ shows how average gas saturation normal to the flow varies with normalized fractional distance. By comparing the average profiles at different times, the authors found that fingers had grown linearly with time. They also noticed that the average saturation profiles and finger growth rates were quite similar for two different realizations of the permeability field.

In randomly heterogeneous media there is no need for artificial perturbations to trigger instability. Khataniar and Peters (1992) simulated displacement of oil by water in heterogeneous media using a standard finite-element method. They considered a medium with a log normal permeability distribution characterized by two measures of variability, the Dykstra-Parson $(D P)$ coefficient and a dimensionless spatial correlation length. The 


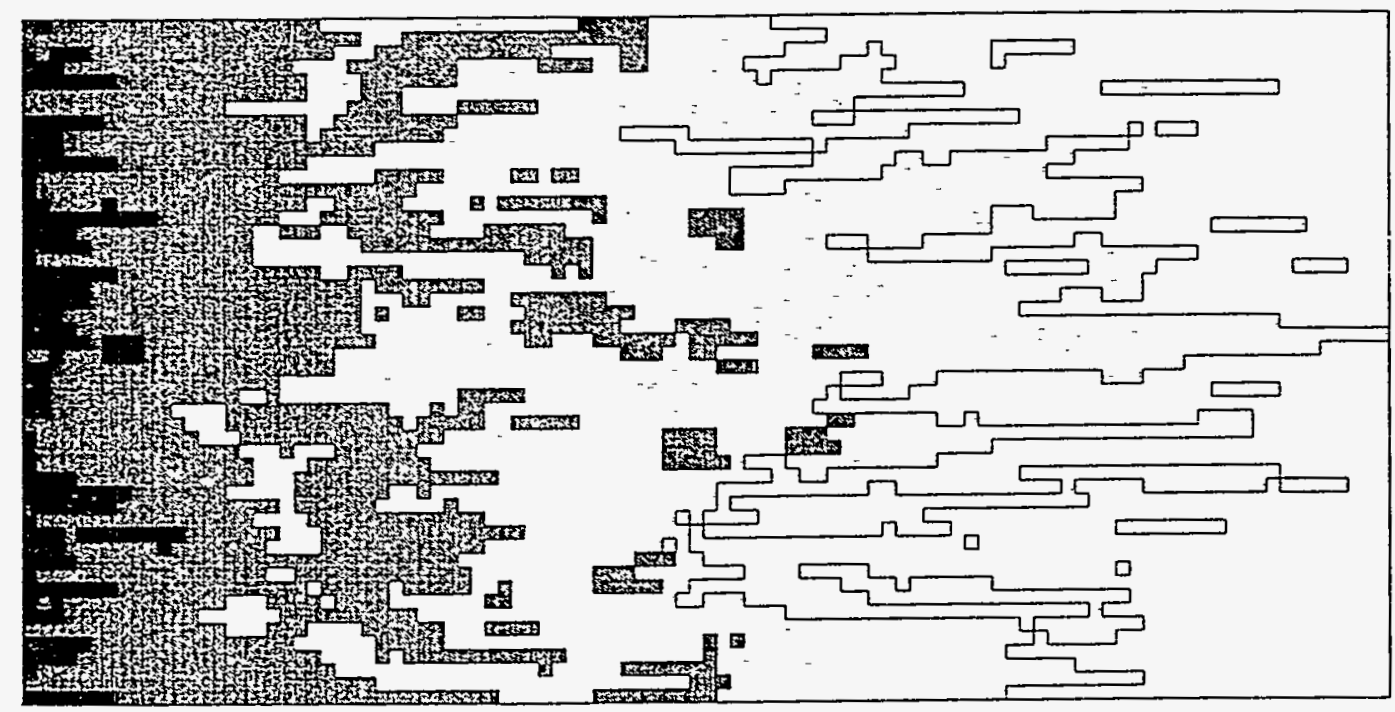

(a)

WATER SATURATION

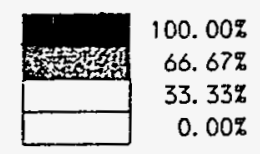

FRACTION OF A PORE VOLUME INJECTED AT BREAKTHROUGH $=0.351$

PERCENTAGE OF GRID BLOCKS CONTAINING WATER AT BREAKTHROUGH $=71.1 \%$

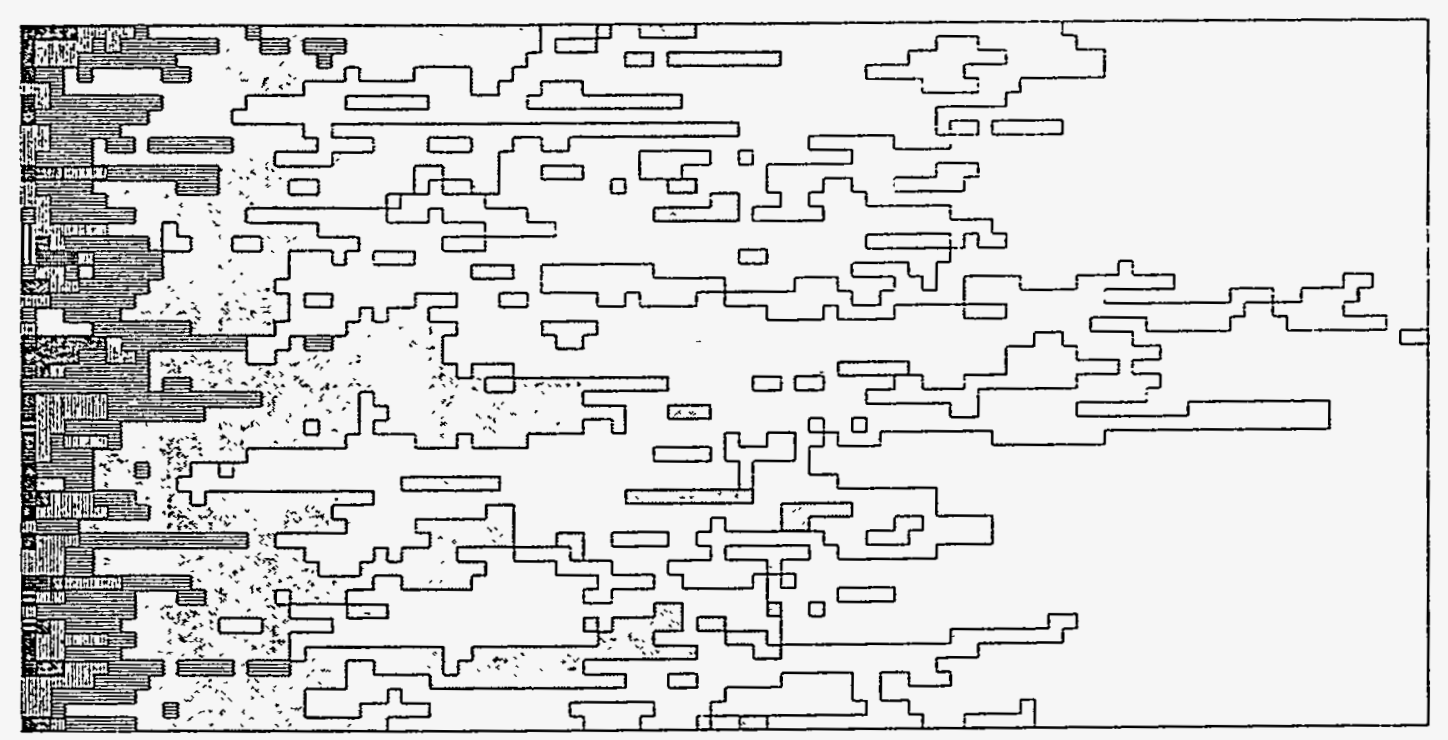

(b)

WATER SATURATTON

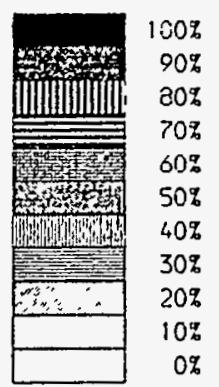

FRACTION OF A PORE VOLUME INJECTED AT BREAKTHROUGH $=0.107$

PERCENTAGE OF GRID BLOCKS CONTAINING WATER AT BREAKTHROUGH $=63.2 \%$

Figure 4.14. Water saturations at breakthrough during displacement of oil by combined finite difference and Monte Carlo simulations: (a) $M=8$, (b) $M=99$ (after Hughes and Murphy, 1988). 


\section{Saturation}

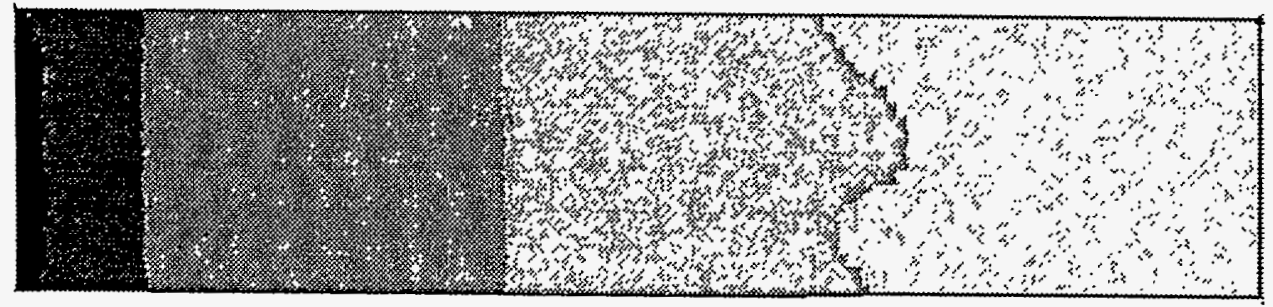

(a)

\section{Saturation}

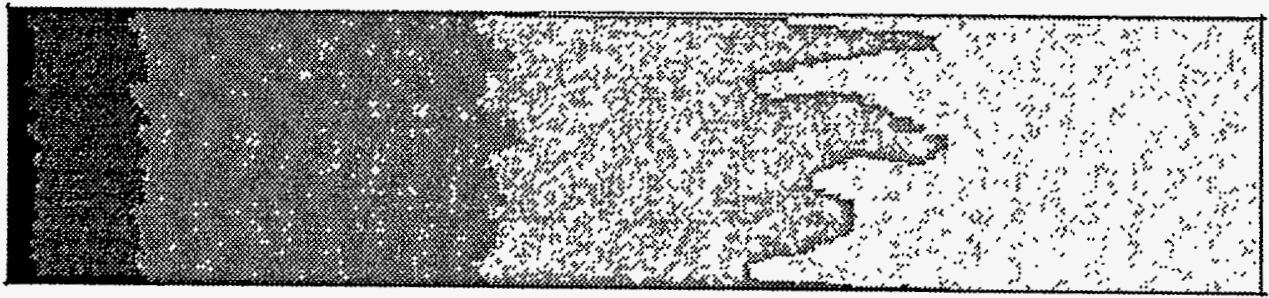

(b)

Figure 4.15. Flux-corrected simulation of oil displacement by water at $M=10$ and (a) moderate capillary pressure, (b) tenfold lower capillary pressure (after Christie, 1989).

(b)

(a)
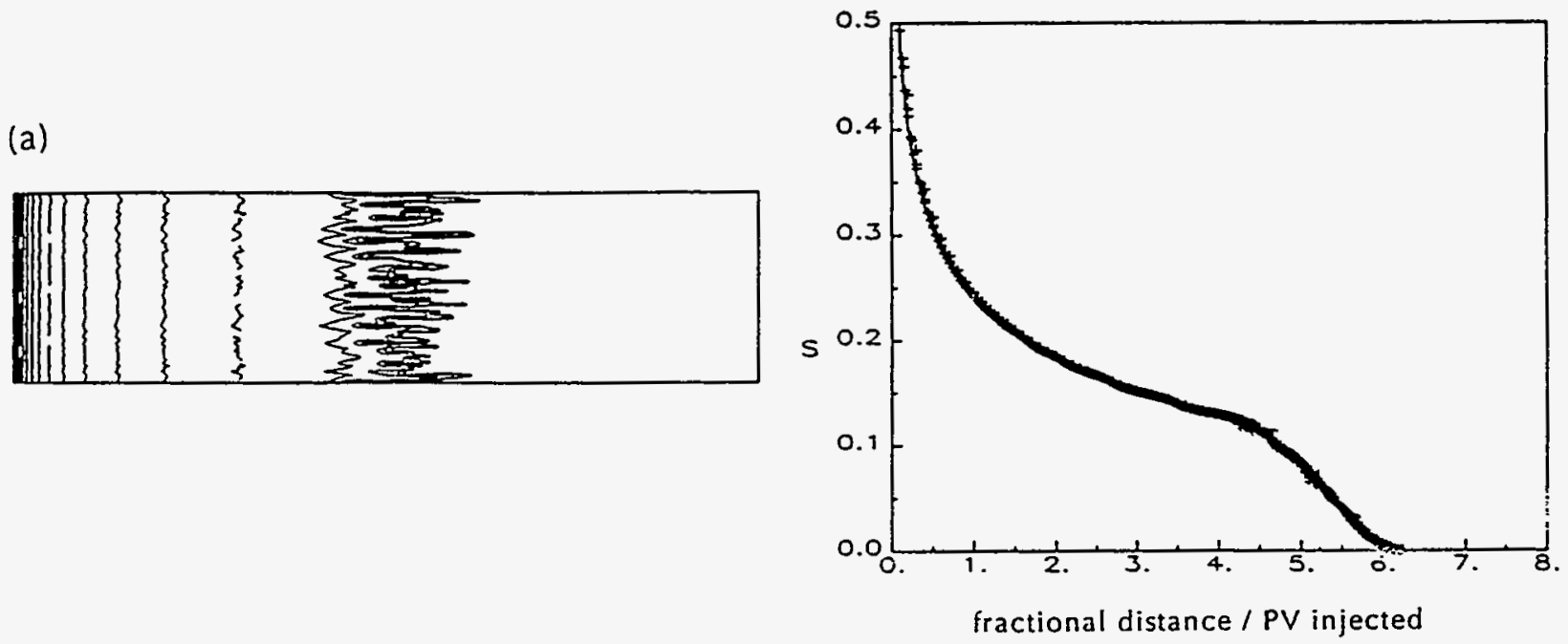

Figure 4.16. Immiscible displacement of oil by gas: (a) gas saturation contours after displacement of 0.1 pore volumes and (b) average gas saturation (after Blunt et al., 1994). 
former is defined as (Dykstra and Parson, 1950)

$$
D P=1-\frac{q_{16}}{q_{50}}
$$

where $q_{16}$ and $q_{50}$ are the $16^{\text {th }}$ and $50^{\text {th }}$ percentiles of the permeability distribution function, respectively. It is related to the standard deviation, $S V$, via

$$
S V=-\ln (1-D P) \text {. }
$$

Khataniar and Peters generated a single random permeability field of twelve combinations of the following parameters: $D P=0.01,0.55,0.89, \lambda_{x}=0.0,0.2,0.7,2.0$ where $\lambda_{x}$ is longitudinal spatial correlation length normalized by the length of the flow field, and $\lambda_{y}=$ 0.2 where $\lambda_{y}$ is the normalized transverse spatial correlation length. Figure 4.17 shows water saturation profiles in all twelve media after injection of one pore volume. The simulations were done in a domain of length $120 \mathrm{~m}$ and width $60 \mathrm{~m}$ at a viscosity ratio of 100 in the absence of gravity and capillarity. The domain was overlain by a grid of 40 elements in the longitudinal direction and 60 elements in the transverse direction. When $D P=0.01$, longitudinal correlation has no significant effect on the front which remains stable at all $\lambda_{x}$. When $D P=0.55$, fingering occurs. In the absence of spatial correlation $\left(\lambda_{x}=0.0\right)$, numerous low-amplitude fingers develop. As $\lambda_{x}$ increases, there is a tendency for a few fingers to grow considerably at the expense of others. At high $\lambda_{x}$, the dominant fingers occupy distinct zones of high permeability. When $D P=0.89$, these same fingers become narrower and longer. It is thus clear that both the variance and the longitudinal autocorrelation of random log permeability variations contribute to the development of fingers along preferential flow paths, thereby reinforcing the effect of hydrodynamic instability.

Figure 4.18 shows plots of fractional recovery ratio between fluid breakthrough and inflow versus pore volume of injected fluid for various $D P$ and $\lambda_{x}$. When $D P=$ 0.01 (Figure 4.18a), fractional recovery is independent of $\lambda_{x}$. When $D P$ increases to 0.89 (Figure $4.18 \mathrm{~b}$ ), fractional recovery declines as $\lambda_{x}$ increases. The effects of $D P$ at a given $\lambda_{x}$ are shown in Figure $4.18 \mathrm{c}$ and $d$. In the absence of correlation (Figure 4.18c), DP has only a minor effect on fractional recovery. When $\lambda_{x}=2.0$ (Figure $4.18 \mathrm{~d}$ ), fractional recovery declines as $D P$ goes up.

The effect of medium heterogeneity on fingering has also been investigated in the 

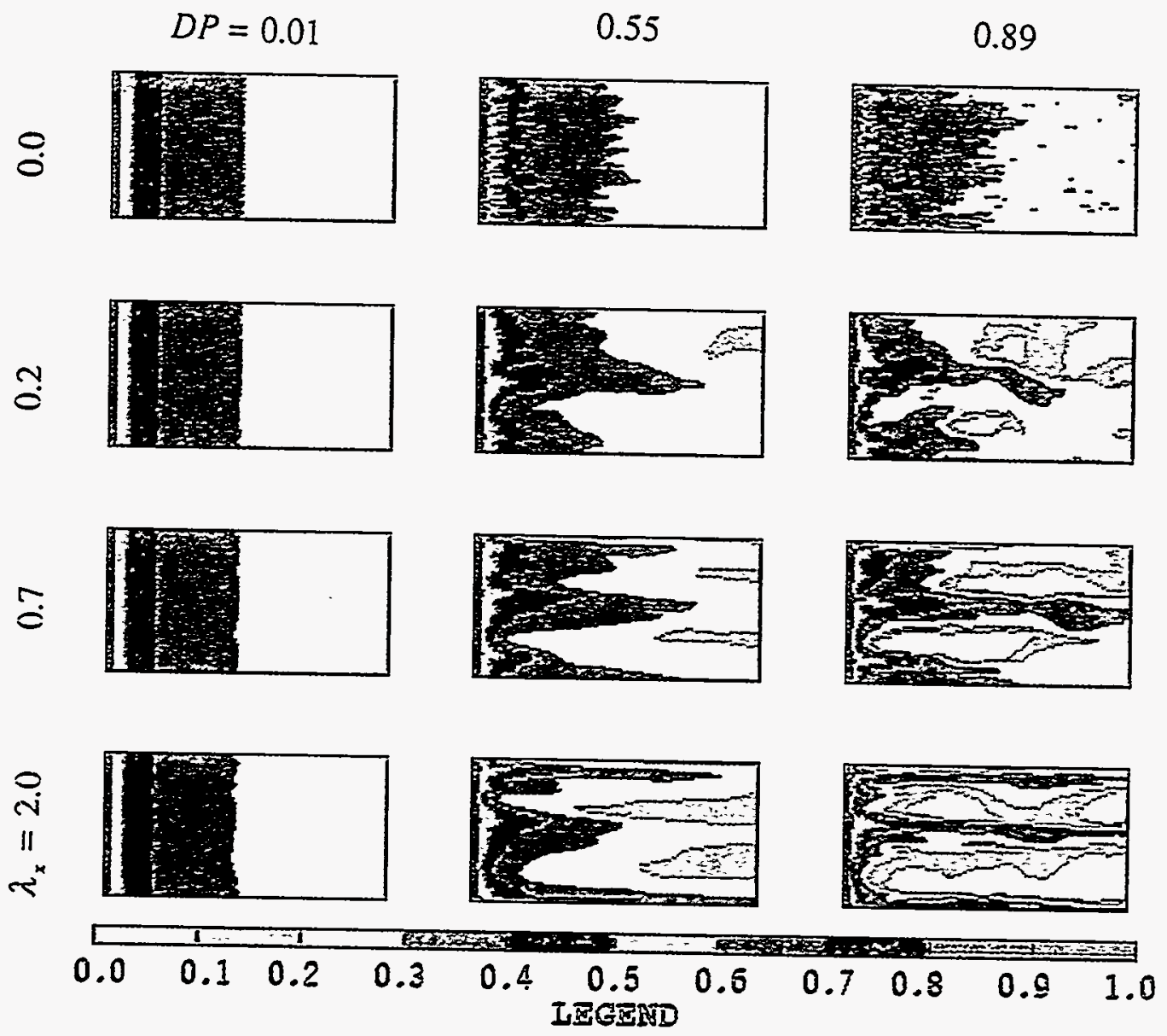

Figure 4.17. Effect of $D P$ and spatial correlation of log permeability on water saturation profiles after injection of one pore volume into a randomly heterogeneous medium filled with oil at a viscosity ratio of 100 (after Khataniar and Peters, 1992). 


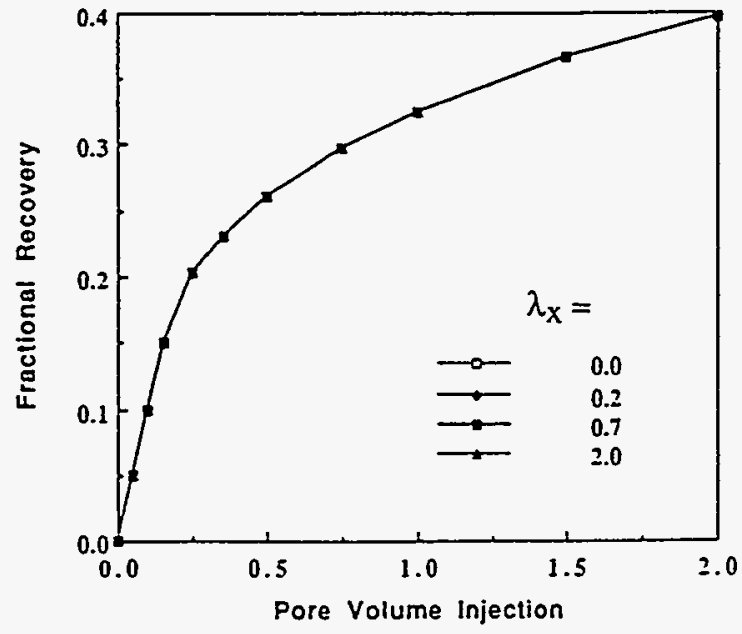

(a)

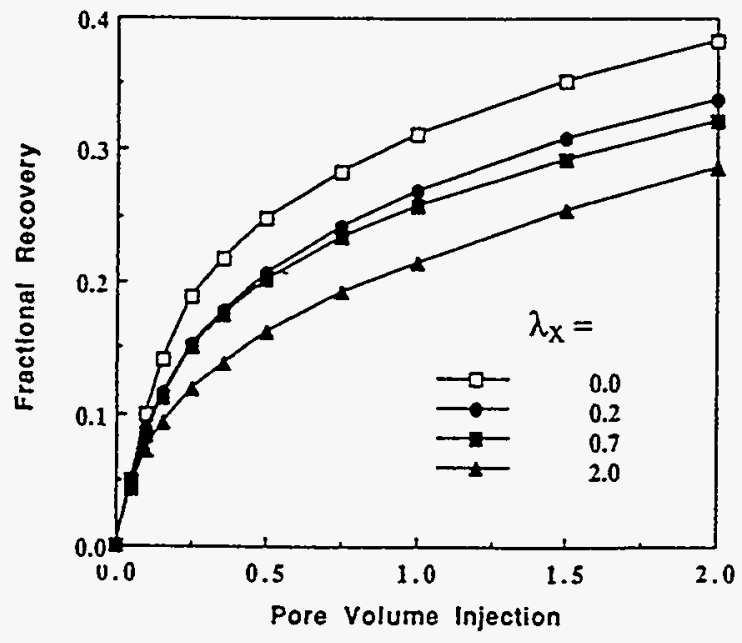

(b)

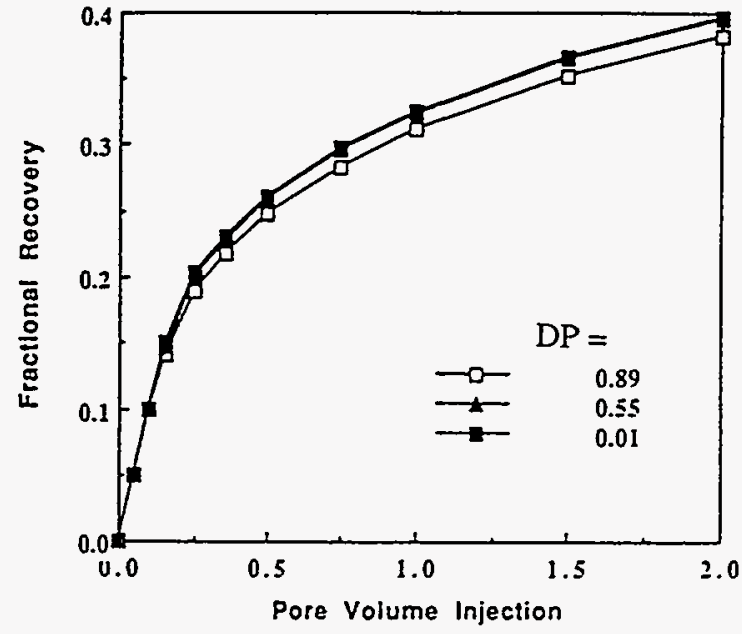

(c)

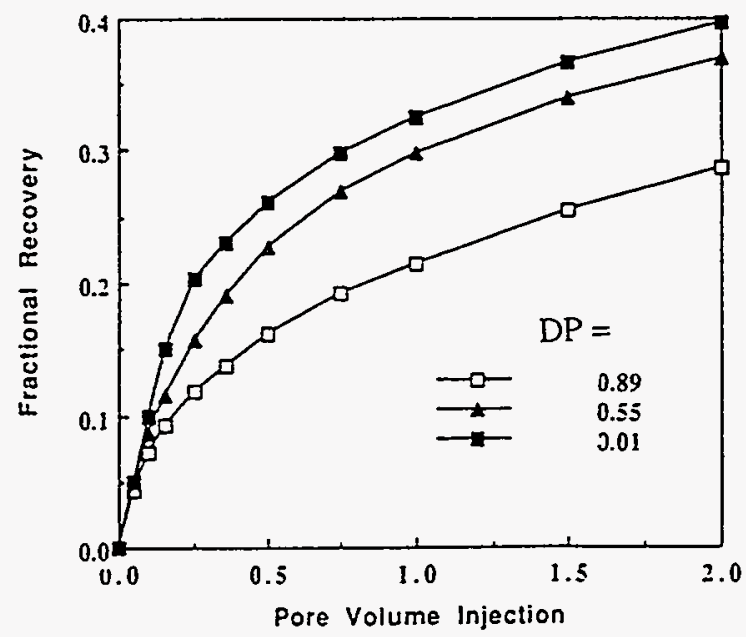

(d)

Figure 4.18. Effect of $D P$ and $\lambda_{x}$ on fractional recovery at $M=100$ : (a) $D P=$ 0.01; (b) $D P=0.89$; (c) $\lambda_{x}=0.0$; (d) $\lambda_{x}=2.0$ (after Khataniar and Peters, 1992). 
context of miscible displacement (e.g., Moissis 1988; Kelkar and Gupta, 1991; Tan and Homsy, 1991; Waggoner et al., 1992; Araktingi and Orr, 1993; Moissis et al, 1993; Tchelepi and Orr, 1993). As the present report focuses on immiscible displacement, these studies fall outside its scope and are not discussed. We mention only the very recent numerical experiments by Hill et al. (1994) on combined water infiltration and solute transport in an unsaturated, heterogeneous soil of the kind found at the Las Cruces Trench site in New Mexico. The two-dimensional experiments covered a vertical section $6 \mathrm{~m}$ deep and $24 \mathrm{~m}$ wide. Measured saturated hydraulic conductivities and initial water contents were interpolated across the section. Water with a nonrective tracer at unit concentration was sprinkled at a uniform rate of $1.16 \times 10^{-5} \mathrm{~cm} / \mathrm{s}$ on the soil surface. Zero flux conditions were assigned along the sides and bottom of the section. Richards' equation was solved by finite differences to yield advective velocities for the domain. The problem of advective-dispersive tracer transport was then solved by means of the fluxcorrected method on a grid with cells of size $10 \times 10 \mathrm{~cm}^{2}$.

Figure 4.19 depicts computed changes in volumetric water content after fifty days of infiltration. It also shows profiles of tracer without and with dispersion (molecular diffusion of $1.16 \times 10^{-5} \mathrm{~cm}^{2} / \mathrm{s}$, isotropic dispersivity of $3 \mathrm{~cm}$ ). The sharp contours of the front in the absence of physical dispersion suggest that numerical dispersion is under control. These contours show fingers which reflect instability of the sprinkled water front. In the model, antecedent water was allowed to move downward ahead of this front. Hence the average depth of the combined (sprinkled and antecedent) moisture front is greater (4 $-5 \mathrm{~m}$ ) than that of the sprinkled front (about $3 \mathrm{~m}$ ). Dispersion is seen to have a stabilizing effect on the solute front, but it of course does not affect the wetting front in this model. 
( $(466 \mathrm{I} " 1012$

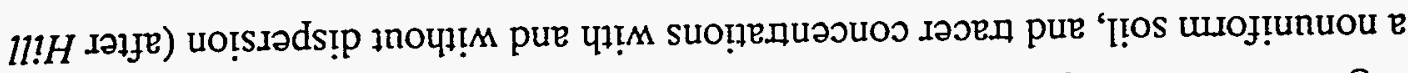

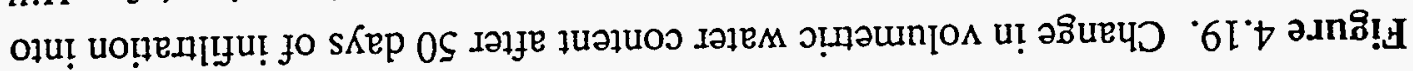

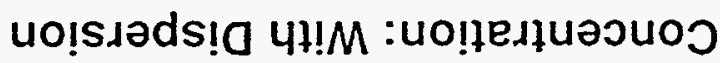

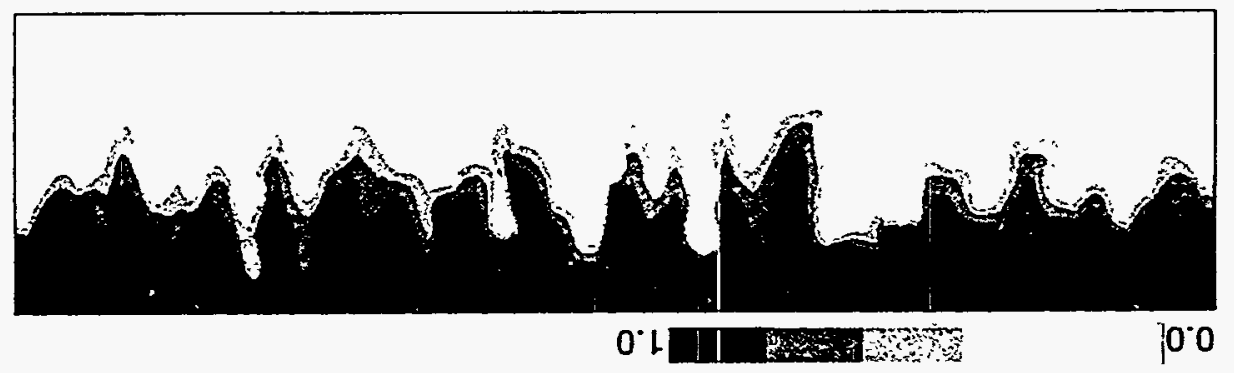

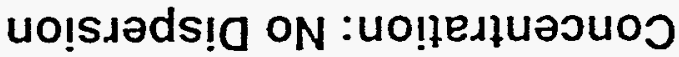

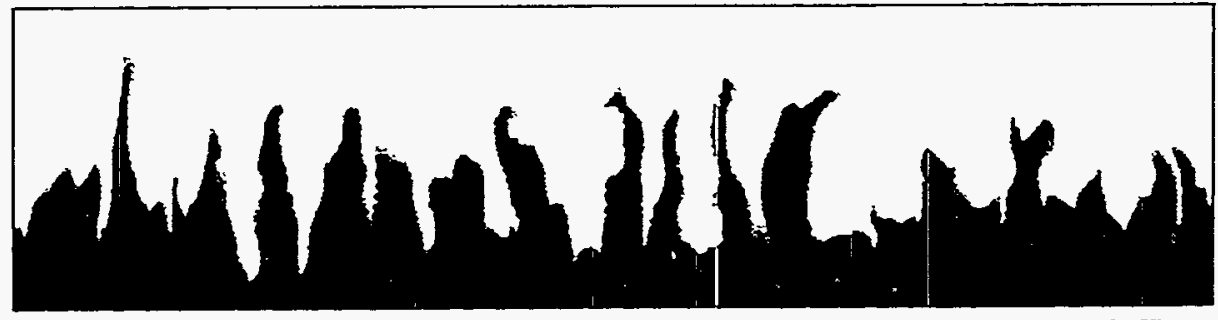

0.4 II

$10 \cdot 0$

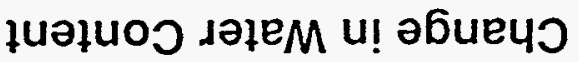

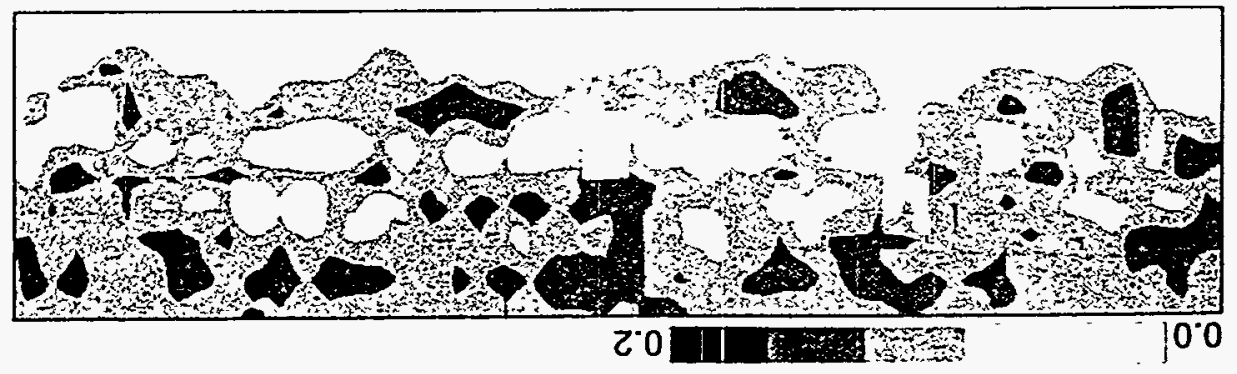




\section{SUMMARY AND RECOMMENDATIONS}

We present below a summary of key findings based on our overview of published experimental, theoretical and numerical work on instability and fingering during immiscible fluid displacement in porous and fractured media, particularly wetting front instability in unsaturated soils and rocks. We also point to some important gaps in existing knowledge which could be potentially narrowed through future research.

\subsection{Summary of Key Findings}

1. Wetting front instability is a commonly occurring phenomenon which has an important effect on fluid flow and contaminant transport in unsaturated soils and rocks. It causes the development of fingers which travel faster than would be anticipated on the basis of Darcy's law. This may cause water and contaminants to reach the water table faster than would otherwise be the case. The phenomenon occurs in both uniform and nonuniform media in response to either ponding or sprinkling at the surface. It also takes place during moisture redistribution in the wake of an infiltration event.

2. Wetting front instability is a special case of interface instability during immiscible fluid displacement in porous and fractured media. The phenomenon is triggered by unfavorable differences between the viscosities and densities of the two fluids across their interface. Viscous forces have a stabilizing influence when a more viscous fluid displaces a less viscous fluid, and a destabilizing influence when a less viscous fluid displaces a more viscous fluid. Both effects increase with the mean propagation speed of the interface. Gravity has a stabilizing influence when the denser fluid is at the bottom, and a destabilizing influence otherwise, regardless of mean flow direction or speed.

When both viscous and gravitational forces act to stabilize the interface, the latter is unconditionally stable. When both of these forces act to destabilize the interface, it is unconditionally unstable. When viscosity is stabilizing and gravity is destabilizing, the interface is stable provided that its mean speed exceeds some critical value. When viscosity is destabilizing and gravity is stabilizing, the interface is stable on condition that its mean speed is below a critical value.

During the downward propagation of a wetting front in an unsaturated soil or rock environment, viscosity acts as a stabilizing force and gravity as a destabilizing force. Hence for instability to occur, the mean speed of front propagation must be less than some 
critical value. For a sharp wetting front with a uniform moisture profile above and below, this critical speed is equal to the ratio between hydraulic conductivity above the front and the drop in water content across it.

3. Surface tension has a stabilizing effect on the interface between two immiscible fluids. Suppose that the interface is perturbed slightly into a composite wave form containing diverse wave lengths (and spatial frequencies) of very small amplitude. If the interface is unstable and there is no surface tension, the amplitudes of all these perturbations (incipient fingers) can initially grow at rates that increase as their wavelengths decrease (frequencies increase). If surface tension is active, only perturbations with wavelengths above some critical value (frequencies below some critical value) can grow, all others decay. The critical wavelength increases with surface tension (for a sharp wetting front with a uniform moisture profile above and below, it decreases as the drop in water content across the front increases). The fastest growing perturbation has a wavelength that exceeds critical by a constant factor. The corresponding fingers are thought to appear first in an experiment and to dominate their neighbors. Smaller fingers often coalesce into one or more dominant fingers.

4. Under uniform mean flow in homogeneous media, established fingers tend to elongate linearly with time. Their rate of elongation increases with mean flow rate and viscosity contrast but decreases with surface tension.

5. Shielding occurs when larger fingers outgrow their smaller neighbors and spread laterally to inhibit their growth. The widening of dominant fingers by shielding reduces surface tension which may render them unstable. This, in turn, may cause the fingers to split (bifurcate) at their tips into narrower branches which are stable due to increased surface tension. Shielding then favors one of these branches which widens, becomes unstable, and so on. The cycle of shielding, spreading and splitting may repeat itself periodically. It is not commonly observed when wetting fronts propagate downward through unsaturated media.

When capillary action is weak and/or mean flow rate is high, bifurcation may continue to yield a contorted, dendritic interface with a randomly fractal geometry.

6. Theoretical considerations published earlier by one of us suggest that, in a Hele-Shaw cell (or ideal fracture with smooth impermeable walls separated by a constant aperture) upon the onset of instability, the interface is generally not perpendicular (and fingers are generally not parallel) to the direction of mean flow unless the latter coincides 
with the dip of the cell (or fracture). We have shown theoretically in this report that, in a three-dimensional porous medium upon the onset of instability, the interface is generally not perpendicular (and fingers are generally not parallel) to the mean direction of flow unless the latter is vertical. This is contrary to Chuoke et al. (1959) who allowed mean flow to be other than vertical but conjectured that the interface would remain at right angle to this flow vector.

7. Spatial variations in medium permeability are conducive to the preferential development of fingers along paths of least resistance to flow. Instability in such heterogeneous media may occur under conditions that would not be favorable for the development of fingers in equivalent uniform media. This is true on the pore scale, on the laboratory scale, and on the field scale where preferential flow of water and solutes is commonly observed. A wetting front becomes unstable as it descends from a fine-grained into a coarse-grained soil. The same happens as it reaches a critical depth when permeability increases systematically downward. In soils that are wetted nonuniformly (say by antecedent fingers), new fingers tend to move preferentially through the wetted regions because these pose the least resistance to flow.

8. A sharp wetting front is more prone to become unstable than a diffuse front; conditions which cause instability in a poorly-graded (usually coarse) soil may therefore give rise to a stable front in a well graded (usually fine) soil. Other factors which contribute to the development of unstable wetting fronts include water repellency of the soil and compression of air ahead of the front.

9. Issues concerning the onset of instability are often amenable to first-order mathematical analysis by linearization. As fingers grow, their behavior becomes so strongly nonlinear that numerical analysis is usually required. Numerical methods that have proven capable of simulating the development of fingers under select conditions include point vortex and vortex-in-cell techniques, random walk models, pore-scale percolation models, Eulerian-Lagrangian methods, and the flux corrected scheme. Among these, the latter two approaches seem better suited for the simulation of unstable fluid interfaces in heterogeneous media than the rest.

\subsection{Some Gaps in Existing Knowledge}

Much is yet to be learned about various aspects of instability and finger development during immiscible displacement. We believe that the effect of soil and rock heterogeneities on the instability of wetting fronts merits special attention in the context of 
nuclear waste storage in unsaturated geologic environments. In particular, little is presently known about the extent to which porous rocks containing fractures, faults and bedding planes (as is the case at Yucca Mountain in Nevada) may facilitate or retard the development of unstable fingers and their rapid propagation (vertically or otherwise) to the water table. What effects do various parameters which define the spatial variability of rock properties (fracture, fault and bedding plane geometries; mean, variance and correlation scales of permeabilities in diverse lithologic and structural units) have on the onset of instabilities under various antecedent moisture conditions and surface infiltration scenarios on relevant field scales? In what directions, and at what rates, might fingers propagate after instability has set in? How long, wide and numerous will such fingers be? What will be their locations and spacings? Will they occur exclusively in fractures or will they invade matrix blocks? How is this related to the mass rate of water and solute propagation through the geologic medium? To address the issue of solute transport one may have to consider not only immiscible but also miscible displacement processes such as dispersion, matrix diffusion and sorption. What effect do these have on the stability of solute fronts under unsaturated conditions? How might elevated temperatures in parts of the flow field affect such behaviors? Might condensed water vapors form potentially unstable moisture fronts? What observations and experiments might feasibly help reveal these and related phenomena in the field? Our overview suggests that these questions address realistic problems which have a direct bearing on our understanding of unsaturated fluid flow and contaminant transport in complex geologic media. 


\section{Appendix A (Analysis of Philip, 1975)}

Integration of (3.64) between the limits in (3.65) yields

$$
h(z)=\frac{\psi_{a}+\psi_{f}-L-\psi_{0}}{\int_{0}^{-L} \frac{1}{K_{s}(z)} d z} \int_{0}^{z} \frac{1}{K_{s}(z)} d z+\psi_{0} .
$$

for the potential $h$ above a Green and Ampt (1911) wetting front. Let $\psi$ be the pressure head defined via $h=\psi+z$. Then the pressure gradient immediately above the wetting front is

$$
G=\left.\frac{\partial \psi}{\partial z}\right|_{z=-L}=\left.\frac{\partial h}{\partial z}\right|_{z=-L}-1=\frac{\psi_{a}+\psi_{f}-L-\psi_{o}}{K_{s}(-L) \int_{0}^{-L} \frac{1}{K_{s}(z)} d z}-1
$$

In term of the depth variable $\xi=-z$, this can be rewritten as

$$
G=-\frac{\psi_{a}+\psi_{f}-L-\psi_{o}-L}{K_{s}(L) \int_{0}^{L} \frac{1}{K_{s}(\xi)} d \xi}-1
$$

where now $K_{s}$ is a function of $\xi$.

Let $\psi^{\prime}$ be the pressure head above the wetting front in the perturbed field and designate the perturbation in pressure head by $\Psi, \Psi=\psi^{\prime}-\psi$. According to Philip, each harmonic of the perturbed field satisfies

$$
\nabla \cdot\left(K_{s} \nabla \Psi\right)=0
$$

subject to the boundary conditions

$$
z=0, \quad \Psi=0
$$

and, to first order,

$$
z=-L, \Psi=-\left(G+\frac{M^{2} T}{\rho g}\right) \varepsilon \sin (n x+\tau) \sin (m y+\delta)
$$

where $T$ is surface tension at the interface, $M^{2}=n^{2}+m^{2}$, and $\Delta \theta=\theta_{s}-\theta_{i}$. The first boundary condition is the requirement that the disturbance vanishes at the soil surface. 
The second boundary condition has two components. The first component comes from the fact that $\psi^{\prime}=\psi(-L)$ at the disturbed wetting front, not at $z=-L$. To first order in $\varepsilon$, this component is

$$
\begin{aligned}
\Psi_{1} & =\psi^{\prime}(-L)-\psi(-L)=-\left.\Theta \frac{\partial \psi}{\partial z}\right|_{z=-L} \\
& =-G \varepsilon \sin (n x+\tau) \sin (m y+\delta)
\end{aligned}
$$

where $\Theta$ is defined in (3.76). The second component results from the fact that the perturbation superposes a curvature on the original planar wetting front and produces a perturbation in capillary pressure, $\Delta \psi_{f}$. To first order in $\varepsilon$, Philip (1975) wrote

$$
\begin{aligned}
\Psi_{2} & =\Delta \psi_{f}=\frac{T}{\rho g}\left(\frac{\partial^{2} \Theta}{\partial x^{2}}+\frac{\partial^{2} \Theta}{\partial y^{2}}\right) \\
& =-\frac{M^{2} T}{\rho g} \varepsilon \sin (n x+\tau) \sin (m y+\delta)
\end{aligned}
$$

As discussed in Section 2.2, White et al. (1976) proposed replacing the surface tension coefficient $T$ by an effective surface tension coefficient $T^{*}=T / \Delta \theta$ for infiltration in porous media. We adopt this replacement in the following.

The solution of (A.4) subject to (A.5) and (A.6) is

$$
\Psi=-\left(G+\frac{M^{2} T}{\Delta \theta \rho g}\right) \varepsilon \sin (n x+\tau) \sin (m y+\delta) \Omega(z)
$$

where $\Omega(z)$ satisfies

$$
\frac{1}{K_{s}} \frac{d}{d z}\left(K_{s} \frac{d \Omega}{d z}\right)-M^{2} \Omega=0
$$

subject to

$$
z=0, \Omega=0 ; z=-L, \Omega=1
$$

The velocity of the front, $v$, due entirely to the perturbed pressure field is

$$
v=-\left.\frac{K_{s}(-L)}{\Delta \theta} \frac{\partial \Psi}{\partial z}\right|_{z=-L}
$$


where $K_{s}$ is a function of $z$. It follows from this and (A.9) that

$$
\nu=-\frac{K_{s}(-L)}{\Delta \theta}\left(G+\frac{M^{2} T}{\Delta \theta \rho g}\right) \varepsilon \sin (n x+\tau) \sin (m y+\delta) \zeta
$$

where $\zeta$ is $-d \Omega / d z$ evaluated at $z=-L$. From (A.10) it follows that, when $K_{s}>0$, $\Omega(z)$ can have neither a positive maximum nor a negative minimum. This together with (A.11) implies that $\Omega(z)$ must be monotonically decreasing and so $\zeta>0$. The velocity of the front due to the perturbation can also be derived from (3.76) as

$$
\nu=\frac{d \varepsilon}{d t} \sin (n x+\tau) \sin (m y+\delta) .
$$

Combining (A.13) and (A.14) yields

$$
\frac{d \varepsilon}{d t}=-\frac{K_{s}(-L)}{\Delta \theta}\left(G+\frac{M^{2} T}{\Delta \theta \rho g}\right) \zeta \varepsilon .
$$

This equation is subject to the condition $\varepsilon\left(t_{o}\right)=\varepsilon_{o}$, hence the solution for $t>t_{o}$ is

$$
\varepsilon(t)=\varepsilon_{o} \exp \left(\int_{t_{o}}^{t} \vartheta d t\right)
$$

where

$$
\vartheta=-\frac{K_{s}(-L)}{\Delta \theta}\left(G+\frac{M^{2} T}{\Delta \theta \rho g}\right) \zeta
$$




\section{Appendix B (Analysis of Parlange and Hill, 1976)}

To obtain an expression for $\Gamma$ in (3.96), Parlange and Hill (1976) considered a cylindrical wetting front moving radially from a cylindrical cavity of radius $r_{c}$ under constant flux $Q$ per radian and unit length of cylinder. In the absence of diffusion, the front is discontinuous and moves at speed $\dot{r}_{o}$ where $r_{o}$ is distance from the axis and the dot designates time derivative. Since the flux at $r_{o}$ is $Q / r_{o}$, it follows that

$$
r_{o} \dot{r}_{o}=Q / \Delta \theta
$$

where $\Delta \theta=\theta_{s}-\theta_{i}, \theta_{s}$ being water content behind the front, $\theta_{i}$ ahead of the front. For a diffusing front, the movement of water is governed by

$$
\frac{\partial \theta}{\partial t}=\frac{1}{r}\left[\frac{\partial}{\partial r}\left(r D \frac{\partial \theta}{\partial r}\right)\right]
$$

subject to the boundary conditions

$$
\begin{gathered}
\left.\left(r D \frac{\partial \theta}{\partial r}\right)\right|_{r=r_{c}}=-Q ; \quad t>0 \\
\theta=\theta_{i} \text { at } t=0 ; \quad \theta=\theta_{i} \text { at } r \rightarrow \infty
\end{gathered}
$$

where $D$ is soil diffusivity. The solution to (B.2) and (B.3) is given approximately by (Parlange, 1973; Sawhney and Parlange, 1976)

$$
\ln \left(r_{i} / r_{s}\right)=\frac{1}{Q} \int_{\theta_{1}}^{\theta_{s}} D d \theta
$$

where $r_{i}$ and $r_{s}$ are the values of $r$ corresponding to $\theta=\theta_{i}$ and $\theta=\theta_{s}$, respectively. The approximation is valid provided one can ignore terms of order

$$
\epsilon=\frac{\int_{\theta_{i}}^{\theta_{s}}\left(\theta_{s}-\theta\right) D d \theta}{\int_{\theta_{i}}^{\theta_{s}} \theta_{s} D d \theta}
$$

compared to other terms. The magnitude of $\epsilon$ is of order 0.1 for many soils. Assuming that front width is small compared to the wave length, $r_{i}-r_{s} \ll r_{s}$, one can replace $\ln \left(r_{i} / r_{s}\right)$ by $\left(r_{i}-r_{s}\right) / r_{s}$ and interchange $r_{i}, r_{s}$, and $r_{o}$ everywhere except in differences. 
Differentiating (B.4) with respect to time and considering (B.1) yields

$$
\dot{r}_{i}-\dot{r}_{s} \approx \frac{1}{r_{i} \Delta \theta} \int_{\theta_{i}}^{\theta_{s}} D d \theta
$$

where $\dot{r}_{i}$ and $\dot{r}_{s}$ represent the velocities of $r_{i}$ and $r_{s}$, respectively. Hence the velocity of the saturated front boundary, $\dot{r}_{s}$, is less than what it would have been in the absence of diffusion, $\dot{r}_{i}$, by $r_{i}^{-1}(\Delta \theta)^{-1} \int_{\theta_{i}}^{\theta_{s}} D d \theta$. Equation (B.6) is comparable to (3.96) where $v$ represents the velocity with diffusion and $U$ the velocity without diffusion. The comparison implies that, since $r_{1}=r_{i}$, and $r_{2}^{-1}=0$ in the present case, then

$$
\Gamma=\frac{1}{\Delta \theta} \int_{\theta_{i}}^{\theta_{s}} D d \theta .
$$

When terms of order $\epsilon$ are small, the sorptivity is given approximately by (Sawhney and Parlange, 1976)

$$
S^{2} \approx 2 \Delta \theta \int_{\theta_{i}}^{\theta_{s}} D d \theta
$$

and $\Gamma$ can be rewritten as

$$
\Gamma=\frac{S^{2}}{2(\Delta \theta)^{2}}
$$




\section{REFERENCES}

Adler, P. M., Porous Media: Geometry and Transport, Butterworth-Heinemann, Boston, Mass., 1992.

Araktingi, U. G. and F. M. Orr, Viscous fingering in heterogeneous porous media, SPE Advanced Technology Series 1, 71-80, 1993.

Baker, R. S. and D. Hillel, Laboratory tests of a theory of fingering during infiltration into layered soils, Soil Sci. Soc. Amer. J., 54, 20-30, 1990.

Baker, R. S. and D. Hillel, Observations of fingering behavior during infiltration into layered soils, Proceeding of the National Symposium on Preferential Flow, Amer. Soc. Agr. Eng., Chicago, IL, USA, 87-99, 1991.

Baptista, A. M., Solution of advection-dominated transport by Eulerian-Lagrangian methods using the backward methods of characteristics, Ph. D. thesis, Dept. of Civil Eng., M. I. T., 1987.

Bensimon, D., L. P. Kadanoff, S. Liang, B. I. Shraiman and C. Tang, Viscous flows in two dimensions, Rev. Mod. Phys., 58, 977-999, 1986.

Birkhoff, G., Taylor instability and laminar mixing, Los Alamos Sci. Lab. Rep. La-1862, 1954.

Blunt, M. J., J. W. Baker, B. Rubin, M. Mansfield, I. D. Culverwell and M. A. Christie, Predictive theory for viscous fingering in compositional displacement, SPE Reservoir Engineering, 73-80, Feb., 1994.

Bond, R. D., The influence of the microfloria on the physical properties of soils, II. Field studies on water repellent sands, Aust. J. Soil Res., 2, 123-131, 1964.

Borie, J. P. and D. L. Book, Flux-corrected transport, I, SHASTA, A fluid transport algorithm that works, J. Comp. Phys., 11, 38-54, 1973.

Borie, J. P. and D. L. Book, Flux-corrected transport, III, Minimal-error FCT algorithms, J. Comp. Phys., 20, 397-431, 1976.

Brock, D. C. and F. M. Orr, Flow visualization of viscous fingering in heterogeneous porous media, Proc. 1991 SPE Annual Tech. Conf. and Exhib., Dallas, TX, 211-222, Oct. 6-9, 1991.

Celia, M. A., T. F. Russell, I. Herrera and R. E. Ewing, An Eulerian-Lagrangian localized adjoin method for the advection-diffusion equation, Adv. Water Res., 13, 187-206, 1990.

Chandler, R., J: Koplic, K. Lerman and J. F. Willemsen, Capillary displacement and percolation in porous media, J. Fluid Mech., 119, 249-267, 1982. 
Chang, W-L, J. W. Biggar and D. R. Nielsen, Fractal description of wetting front instability in layered soil, Water Resources Res., 30, 125-132, 1994.

Christiansen, J. P., Numerical simulation of hydrodynamics by the method of point vortices, J. Comp. Phys. 13, 363-379, 1973.

Christie, M. A., High-resolution simulation of unstable flows in porous media, SPE Reservoir Engineering, 297-303, Aug., 1989.

Chuoke, R. L., P. van Meurs and C. van der Poel, The instability of slow, immiscible, viscous liquid-liquid displacements in permeable media, Trans. AIME, 216, 188-194, 1959.

Croissant, R., Dévelopment des instabilités en milieu poreux-influence de la pression capillaire, paper 12 presented at the 1968 111e Colloque de 1'ARTFP, Pau, France, Sept. 23-26, 1968 (in Franch).

Davies, G. M., A. H. Muggeridge and A. D. W. Jones, Miscible displacements in a heterogeneous rock: Detailed measurements and accurate predictive simulation, Proc. 1991 SPE Annual Tech. Conf. and Exhib., Dallas, TX, 223-237, Oct. 6-9, 1991.

DeBano, L. F., Water repellent soils: a worldwide concern in management of soil and vegetation, Agric. Sci. Rev., 7, 11-18, 1969.

Deecke, W., Einige Beobachtungen am Sandstrande, Centralbl, für Mineral, Geol. und Paläont., 721-727, 1906.

de Haan, H. J., Effect of capillary forces in the water-drive process, Proc. 5th World Pet. Cong., New York, Sec II, 544-554, 1959.

Diment, G. A. and K. K. Watson, Stability analysis of water movement in unsaturated porous materials, 2, Numerical studies, Water Resour. Res., 19, 1002-1010, 1983.

Diment, G. A. and K. K. Watson, Stability analysis of water movement in unsaturated porous materials, 3, Experimental studies, Water Resour. Res., 21, 979-984, 1985.

Diment, G. A., K. K. Watson and P. J. Blennerhassett, Stability analysis of water movement in unsaturated porous materials, I. Theoretical Considerations, Water Resour. Res., 18, 1248-1254, 1982.

Douglas, J. Jr. and T. F. Russell, Numerical methods for convection-dominated diffusion problems based on combining the method of characteristics with finite element or finite difference procedures, SIAM J. Num. Anal., 19, 871-885, 1982.

Douglas. J. K. and Y. Yiang, Numerical simulation of immiscible flow in porous media based on combining the method of characteristics, in Numerical Simulation in Oil Recovery, M. F. Wheeler (ed.), 119-132, 1988

Dullien, F. A. L., Porous media: Fluid transport and pore structure, Academic Press, Inc., second edition, 1992.

Dykstra, H. and R. L. Parsons, The prediction of oil recovery by water flood, in Secondary Recovery of Oil in the U. S., Principles and Practice, second edition, API, Dallas, 160174, 1950. 
Ewing, R. E., Simulation of multiphase flows in porous media, Transport in Porous Media, 6, 479-499, 1991.

Ewing, R. E., T. F. Russell and M. F. Wheeler, Convergence analysis of an approximation of miscible displacement in porous media by mixed finite elements and a modified method of characteristics, Comp. Math. Apple. Mech. Engng., 47, 73-92, 1984.

Feder, J., Fractals, Plenum Press, 1988

Ferrand, L. A., M. A. Celia and E. S. Wendy, Percolation-based models for pore-to-lab scale calculation in multifluid porous media, in Dynamics of fluids in hierarchical porous media, J. H. Cushman (ed.), 463-480, Academic Press, 1990.

Flury, M., H. Flühler, W. A. Jury and J. Leuenberger, Susceptibility of soils to preferential flow of water: A field study, Water Resour. Res., 30, 1945-1954, 1994.

Gees, R. A. and A. K. Lyall, Erosional sand columns in dune sand, Cape Sable Island, Nova Scotia, Canada, Canadian J. of Earth Science, 6, 344-347, 1969.

Ghodrati, M. and W. A. Jury, A field study using dyes to characterize preferential flow of water, Soil Sci. Soc. Am. J., 54, 1558-1563, 1990

Glass, R. J., Modeling gravity-driven fingering in rough-walled fractures using modified percolation theory, Proc. 4th Int. Conf. on High Level Radioactive Waste Management, Las Vegas, Nevada, 2042-2052, April 26-30, 1993.

Glass, R. J. and T. S. Steenhuis, Factors influencing infiltration flow instability and movement of toxics in layered sandy soils in layered sandy soils, Tech. Pap. 84-2508, Am. Soc. of Agric. Eng., St. Joseph, Mich., 1984.

Glass, R. J., J.-Y. Parlange and T. S. Steenhuis, Water infiltration in layered soils where a fine textured layer overlays a coarse sand, Proc. of the Int. Conf. for Infiltration Development and Application, Jan. 5-9, Hawaii, 1987.

Glass, R. J., T. S. Steenhuis and J. -Y. Parlange, Wetting front instability as a rapid and far-reaching hydrologic process in the vadose zone, J. Contam. Hydrol., 3, 207-226, 1988.

Glass, R. J., J. -Y. Parlange and T. S. Steenhuis, Wetting front instability, 1, Theoretical discussion and dimensional analysis, Water Resour. Res., 25, 1187-1194, 1989a.

Glass, R. J., T. S. Steenhuis and J. -Y. Parlange, Wetting front instability, 2, Experimental determination of relationships between system parameters and two-dimensional unstable flow field behavior in initially dry porous media, Water Resour. Res., 25, 1195-1207, 1989 b.

Glass, R. J., T. S. Steenhuis and J. -Y. Parlange, Mechanism for finger persistence in homogeneous, unsaturated, porous media: Theory and verification, Soil Sci., 148, 6070,1989 c.

Glass, R. J., S. Cann, J. King, N. Baily, J. -Y. Parlange, and T. S. Steenhuis, Wetting front instability in unsaturated porous media: A three-dimensional study in initially dry sand, Transport in Porous Media, 5, 247-268, 1990. 
Glass, R. J., J. -Y. Parlange and T. S. Steenhuis, Immiscible displacement in porous media: Stability analysis of three-dimensional, axisymmetric disturbances with application to gravity-driven wetting front instability, Water Resour. Res., 27, 1947$1956,1991$.

Green, W. H. and G. A. Ampt, Studies in soil physics, I, The flow of air and water through soil, J. Agr. Sci., 4, 1-24, 1911.

Gripp, K., Uber Werden und Vergehen von Barchanen an der Nordsee-Küste SchleswigHolsteins, Zeitsch, für Geomorphologia, Neue Folge, Bd. 5, 24-36, Berlin, Germany, 1961.

Gupta, S. P. and R. A. Greenkorn, An experimental study of immiscible displacement with an unfavorable mobility ratio in porous media, Water Resour. Res., 10, 371-374, 1974.

Gupta, S. P., J. E. Varnon and R. A. Greenkorn, Viscous fingering wavelength degeneration in Hele-Shaw models, Water Resour. Res., 9, 1039-1046, 1973.

Hendrickx, J. M. H. and L. W. Dekker, Experimental evidence of unstable wetting fronts in homogeneous non-layered soils, Proceeding of the National Symposium on Preferential Flow, Amer. Soc. Agr. Eng., Chicago, IL, USA, 22-31, 1991.

Hendrickx, J. M. H. and L. W. Dekker and O. H. Boersma, Unstable wetting fronts in water repellent field soils, J. Environ. Qual., 22, 109-118, 1993.

Herrera, I., R. E. Ewing, M. A. Celia, T. F. Russell, Eulerian-Lagrangian localized adjoint methods: The theoretical framework, Num. Methods for Partial Diff. Equ., 9, 431-457, 1993.

Hill, S., Channeling in packed columns, Chem. Eng. Sci., 1, 247-253, 1952.

Hill, D. E. and J. -Y. Parlange, Wetting front instability in layered soils, Soil Sci. Soc. Amer. Proc., 36, 697-702, 1972.

Hill, R. G., K. A. Fisher, M. R. Kirkland and P. J. Wierenga, Application of flux-corrected transport to the Las Cruces Trench site, Water Resour. Res., 30, 2377-2385, 1994.

Hillel, D., Unstable flow in layered soils: A review, Hydrological Processes, 1, 143-147, 1987.

Hillel, D., Unstable Flow: A potentially significant mechanism of water and solute transport to groundwater, in Water Flow and Solute Transport in Soils, D. Russo and G. Dagan (eds.), 123-135, Springer-Verlag, 1993

Hillel, D. and R. S. Baker, A descriptive theory of fingering during infiltration into layered soils, Soil Sci., 146, 51-56, 1988.

Homsy, G. M., Viscous fingering in porous media, Ann. Rev. Fluid Mech., 19, 271-311, 1987.

Hove, A. O., V. Nilsen and J. Leknes, Visualization of Xanthan flood behavior in core sample by means of X-ray tomography, SPE Reservoir Engineering, 475-480, Nov. 1990. 
Hughes, D. S. and P. Murphy, Use of a Monte Carlo method to simulate unstable miscible and immiscible flow through porous media, SPE Reservoir Engineering, Nov., 1129$1136,1988$.

Jacquard, P. and P. Seguier, Movement de deux fluids en contact dans un milieu poreux, Jour. Mec., 1(4), 367-394, 1962.

Kadanoff, L. P., Simulating hydrodynamics: A pedestrian model, J. Stat. Phys., 39(3/4), 267-283, 1985.

Kelkar, M. and S. P. Gupta, A numerical study of viscous instabilities and scaling considerations, SPE Reservoir Engineering, February, 121-128, 1991.

Khataniar, S and E. J. Peters, The effect of reservoir heterogeneity on the performance of unstable displacements, J. of Petrol. Sci. and Engng., 7, 263-281, 1992.

King, M. J., W. B. Lindquist and L. Reyna, Stability of two dimensional immiscible flow to viscous fingering, Research and Development Report, New York University, 36pp, 1985.

Kueper, B. H. and E. O. Frind, An overview of immiscible fingering in porous media, J. Contam. Hydrol., 2, 95-110, 1988.

Kung, K. J. S., Preferential flow in a sandy vadose zone: 1 . Field observation, Geoderma, 46, 51058, 1990.

Lamb, H., Hydrodynamics, 6th ed., Cambridge University Press, 1932.

Lenormand, R. and S. Bories, Description d'un mécanisme de connexion de liaisons destiné à létude du drainge avec piégega en millieu, C. R. Acad. Sci. Paris 291B, 279$280,1980$.

Lenormand, R., E. Touboul and C. Zarcone, Numerical models and experiments on immiscible displacements in porous media, J. Fluid Mech., 189, 165-187, 1988.

Liang, S., Random-walk simulations of flow in Hele-Shaw cells, Phys. Rev. A, 33, 2663$2674,1986$.

Liu, Y., T. S. Steenhuis, J. -Y. Parlange and J. S. Selker, Hysteretic finger phenomena in dry and wetted sands, Proc. of the National Symp. on Preferential Flow, Amer. Soc. Agr. Eng., Chicago, IL, USA, 160-172, 1991.

Maher, J. V., Development of viscous fingering patterns, Phys. Rev. Lett., 54, 1498-1501, 1985.

Måløy, K. J., J. Feder and T. Jåssang, Viscous fingering fractals in porous media, Phys. Rev. Lett., 55, 2688-2691, 1985.

Mandelbrot, B. B., The Fractal Geometry of Nature, W. H. Freeman, New York, 1982.

Markstein, G. H., Experimental and theoretical studies of flame front stability, J. Aeronaut. Sci., 18, 199-209, 1951. 
Maxworthy, T., The nonlinear growth of a gravitational unstable interface in a Hele-Shaw cell, J. Fluid Mech., 177, 207-232, 1987.

Meakin, P., Fractal aggregates in geophysics, Reviews of Geophysics, 29, 317-354, 1991.

Meiburg, E. and G. M. Homsy, Vortex methods for porous media flows, in Numerical Simulation Oil Recovery, M. F. Wheeler (ed.), Springer-Verlag, New York, IMA 11, 199-226, 1988a.

Meiburg, E. and G. M. Homsy, Nonlinear unstable viscous fingers in Hele-Shaw flows. II. Numerical simulation, Phys., Fluids 31, 429-439, 1988 b.

Miller, D. E. and W. H. Gardner, Water infiltration into stratified soil, Soil Sci. Soc. Amer. Proc., 26, 115-119, 1962.

McCormack, P. D. and L. Crane, Physical Fluid Dynamics, Academic Press, New York, 1973.

Moissis, D. E., Simulation of viscous fingering during miscible displacement in nonuniform porous media, Technical Report 88-9, Rice University, 182pp, 1988.

Moissis, D. E., M. F. Wheeler and C. A. Miller, Simulation of miscible viscous fingering using a modified method of characteristics: effects of gravity and heterogeneity, SPE Advanced Tech. Series 1, 62-70, 1993.

Neuman, S. P., An Eulerian-Lagrangian numerical scheme for the dispersion-convection equation using conjugate space-time grids, J. Comp. Phys., 41, 270-294, 1981.

Neuman, S. P., Gas leakage through fractures from storage cavities below the water table due to interface instability, Research Report, 1982.

Neuman, S. P., Adaptive Eulerian-Lagrangian finite element method for advectiondispersion, Int. J. Num. Meth. Engng., 20, 321-337, 1984.

Neuman, S. P. and S. Sorek, Eulerian-Lagrangian method for advection-dispersion, Proc. 4th Int. Conf. Finite Elements in Water Resources, Holz et al. (eds.), Springer-Verlag, $14,14.41-14.68,1982$.

Nicholl, M. J., R. J. Glass and S. W. Wheacraft, Gravity-driven infiltration instability in initially dry nonhorizontal fractures, Water Resour. Res., 30, 2533-2546, 1994.

Nittmann, J., G. Daccord and H. E. Stanley, Fractal growth of viscous fingers: quantitative characterization of a fluid instability phenomenon, Nature, 314, 141-144, 1985.

Park, C. -W. and G. M. Homsy, Two-phase displacement in Hele Shaw cells: theory, J. Fluid Mech., 139, 291-308, 1984.

Park, C. -W. and G. M. Homsy, The instability of long fingers in Hele-Shaw flows, Phys. Fluids, 28, 1583-1585, 1985.

Park, C. -W., S. Gorell and G. M. Homsy, Two-phase displacement in Hele Shaw cells: experiments on viscously driven instabilities, J. Fluid Mech., 141, 275-287, 1984. 
Parlange, J. -Y., Theory of water movement in soils: 10. Cavities with constant flux, Soil Sci., 116, 1-7, 1973.

Parlange, J. -Y. and D. E. Hill, Theoretical analysis of wetting front instability in soils, Soil Sci., 122, 236-239, 1976.

Paterson, L., Radial fingering in a Hele-Shaw cell, J. Fluid Mech., 113, 513-529, 1981.

Paterson, L., Diffusion Limited aggregation and two-fluid displacements in porous media, Phys. Rev. Lett., 52, 1621-1624, 1984.

Paterson, L., V. Hornof and G. Meale, Visualization of a surfactant flood of an oilsaturated porous medium, Soc. Pet. Eng. J., 24, 325-327, 1984.

Pavone, D., Observations and correlations for immiscible viscous-fingering experiments, SPE Reservoir Engineering, May, 187-194, 1992.

Pearl, Z., M. Magaritz and P. Bendel, Nuclear magnetic resource imaging of miscible fingering in porous media, Transport in Porous Media, 12, 107-123, 1993.

Peck, A. J., Moisture profile development and air compression during water uptake by bounded porous bodies: 3. Vertical columns, Soil Sci., 100, 44-51, 1965.

Perkins, T. K. and O. C. Johnston, A study of immiscible fingering in linear models, Soc. Pet. Eng. J., 9, 39-46, 1969.

Peters, E. J. and D. L. Flock, The onset of instability during two-phase immiscible displacement in porous media, Soc. Pet. Eng. J., 21, 249-258, 1981.

Philip, J. R., Stability analysis of infiltration, Soil Sci. Soc. Am. Proc., 39, 1042-1049, 1975.

Pitts, E., Penetration of fluid into a Hele-Shaw cell: the Saffman-Taylor experiment, J. Fluid Mech., 97, 53-64, 1980.

Quade, J. and T. E. Cerling, Stable isotopic evidence for a pedogenic origin of fracturefilling carbonates in Trench 14 near Yucca Mountain Nevada, Science, 250, 1549$1552,1990$.

Raats, P. A. C., Unstable wetting fronts in uniform and nonuniform soils, Soil Sci. Soc. Amer. Proc., 37, 681-685. 1973.

Ritsema, C. J. and W. Dekker, How water moves in a water repellent sandy soil, 2 . Dynamics of fingered flow, Water Resour. Res., 30, 2519-2531, 1994.

Ritsema, C. J., L. W. Dekker, J. M. H. Hendrickx and W. Hamminga, Preferential flow mechanism in a water repellent sandy soil, Water Resour. Res., 29, 2183-2193, 1993.

Saffman, P. G., Exact solutions for the growth of fingers from a flat interface between two fluids in a porous medium or Hele-Shaw cell, Q. J. Mech Appl. Math., 12, 146-150, 1959.

Saffman, P. G., Viscous fingering in Hele-Shaw cells, J. Fluid Mech., 173, 73-94, 1986. 
Saffman, P. G., Selection mechanisms and stability of fingers and bubbles in Hele-Shaw cells, IMA J. of Appl. Mech., 46, 137-145, 1991.

Saffman, P. G. and G. Taylor, The penetration of a fluid into a porous medium or HeleShaw cell containing a more viscous fluid, Proc. Roy. Soc. London, Ser. A 245, 312$329,1958$.

Samani, Z., A. Cheraghi, and L. Willardson, Water movement in horizontally layered soils, J. Irrigation and Drainage Engineering, 115, 449-456, 1989.

Sawhney, B. L. and J. -Y. Parlange, Radial spreading of a water film on the surface of a soil layer - Theory and application to the determination of soil water diffusivity, Soil Sci. Soc. Am. J., 40, 635-639,1976.

Schwille, F., Dense Chlorinated solvents in porous and fractured media: model experiments, Lewis publishers, 1988.

Selker, J., P. Leclerq, J. -Y. Parlange and T. S. Steenhuis, Fingered flow in two dimensions, 1. Measurement of metric potential, Water Resour. Res. 28, 2513-2521, $1992 a$.

Selker, J., J. -Y. Parlange and T. S. Steenhuis, Fingered flow in two dimensions, 2. Predicting finger moisture profile, Water Resour. Res., 28, 2523-2528, $1992 \mathrm{~b}$.

Smith, W. O., Infiltration in sand and its relation to ground water recharge, Water Resour. Res., 3, 539-555, 1967.

Starr, J. L., H. C. DeRoo, C. R. Frink and J. -Y. Parlange, Leaching characteristics of a layered field soil, Soil Sci. Soc. Am. J., 42, 386-391, 1978.

Starr, J. L., J. -Y. Parlange and C. R. Frink, Water and chloride movement through a layered field soil, Soil Sci. Soc. Am. J., 50, 1384-1390, 1986.

Stokes, J. P., D. A. Weitz, J. P. Gollub, A. Dougherty, M. O. Robbins, P. M. Chaikin, and H. M. Lindsay, Interfacial stability of immiscible displacement in a porous media, Phys. Rev. Lett. 57, 1718, 1986.

Tabeling, P., G. Zocchi and A. Libchaber, An experimental study of the Saffman-Taylor instability, J. Fluid Mech., 177, 67-82, 1987.

Tabuchi, T., Infiltration and ensuing percolation in columns of layered glass particles packed in laboratory (In Japanese with English summary), Nogyo Doboku Kenkyu, Bessatsu (Trans. Agr. Eng. Soc., Japan) 1, 13-19, 1961.

Tamai, N., T. Asaeda and C. G. Jeevaraj, Fingering in two-dimensional homogeneous, unsaturated porous media. Soil Sci., 144, 107-112, 1987.

Tan, C. - T. and G. M. Homsy, Viscous fingering with permeability heterogeneity, Phys. Fluids A, 4, 1099-1101, 1991.

Tang, C., Diffusion limited aggregation and the Saffman Taylor problem, Phys. Rev. A, 31, 1977-1979, 1985. 
Tchelepi, H. A. and F. M. Orr, Jr., The interaction of viscous fingering, permeability heterogeneity and gravity segregation in 3D, SPE 25235, 12th SPE Symp. on Reservoir Simulation, New Orleans, Feb. 28 - March 3, 1993.

Tryggvason, G. and H. Aref, Numerical experiments on Hele-Shaw flow with a sharp interface, J. Fluid Mech., 136, 1-30, 1983.

Tryggvason, G. and H. Aref, Finger-interaction mechanisms in stratified Hele-Shaw flow, J. Fluid Mech., 154, 287-301, 1985.

van Dieren, J. W., Organogene Dünenbildung, Eine geomorphologische Analyse der Dünenlandsxhaft der West-Frierischen Insel Terschelling mit pflanzensoziologischen Methoden, Martinus Nijhoff, Den Haag, the Netherlands, 1934.

van Dam, J. C., J. M. H. Hendrickx, H. C. van Ommen, M. H. Bannink, M. TH. van Genuchten and L. W. Dekker, Water and solute movement in a coarse-textured waterrepellent field soil, J. Hydrology, 120, 359-379, 1990.

van Meurs, $\mathrm{P}$, The use of transparent three-dimensional models for studying the mechanism of flow processes in oil reservoirs, Trans., AIME, 210, 295-301, 1957.

van Ommen, H. C., R. Dejksma, J. M. H. Hendrickx, L. W. Dekker, J. Hulshof and M. van den Heuvel, Experimental assesment of preferential flow paths in a field soil, J. of Hydrology, 105, 253-262, 1989.

Waggoner, J. R., J. L. Castillo and L. W. Lake, Simulation of EOR processes in stochastically generated permeable media, SPE Formation Evaluation, 173-180, June, 1992.

Wiggert, J and T. Maxworthy, Modal behavior observed in the evolution of immiscible interface within an injection-forced Hele-Shaw cell, Phy. Rev. E, 47, 1931-1946, 1993.

Witten, T. A. and L. M. Sander, Diffusion-limited aggregation, a kinetic critical phenomenon, Phys. Rev. Lett., 47, 1400, 1981.

Witten, T. A. and L. M. Sander, Diffusion-limited aggregation, Phys. Rev. B, 27, 5686, 1983.

White, I., P. M. Colombera and J. R. Philip, Experimental study of wetting front instability induced by sudden change of pressure gradient, Soil Sci. Soc. Am. J., 40, 824-829, 1976.

White, I., P. M. Colombera and J. R. Philip, Experimental study of wetting front instability induced by gradual change of pressure gradient and by heterogeneous porous media, Soil Sci. Soc. Am. J., 41, 483-489, 1977.

Wilkinson, D., Percolation effects in immiscible displacement, Phys. Rev. A, 34, 1380$1391,1986$.

Wilkinson, D. and J. F. Willemsen, Invasion percolation: a new form of percolation theory, J. Phys. A, 16, 3365-3376, 1983. 
Wooding, R. A. and H. J. Morel-Seytoux, Multiphase fluid flow through porous media, Ann. Rev. Fluid. Mech., 8, 233-276, 1976.

Zalesak, S. T., Fully multidimensional flux-corrected transport algorithms for fluids, J. Comp. Phys., 31, 335-362, 1978. 


\begin{tabular}{|c|c|}
\hline $\begin{array}{l}\text { U.S. NUCLEAR REGULATORY COMMISSION } \\
\text { BIBLIOGRAPHIC DATA SHEET } \\
\text { (See instructions on the reverse) }\end{array}$ & \multirow[t]{2}{*}{$\begin{array}{l}\text { 1. REPORT NUMBER } \\
\text { (Assigned by NRC. Add Vol., Supp., Rev., } \\
\text { and Addondum Numbers, if any.) } \\
\text { NUREG/CR-6308 }\end{array}$} \\
\hline \multirow{4}{*}{$\begin{array}{l}\text { 2. TITLE AND SUBTITLE } \\
\text { An Overview of Instability and Fingering During } \\
\text { Immiscible Fluid Flow in Porous and Fractured Media }\end{array}$} & \\
\hline & 3. DATE REPORT PUBLISHED \\
\hline & \begin{tabular}{|l|r|} 
MONTH & YEAR \\
Apri1 & 1995 \\
\end{tabular} \\
\hline & $\begin{array}{c}\text { 4. FIN OR GRANT NUMBER } \\
\text { L1283 }\end{array}$ \\
\hline $\begin{array}{l}\text { 5. AUTHOR(S) } \\
\text { G. Chen, M. Taniguchi, S. P. Neuman }\end{array}$ & $\begin{array}{l}\text { 6. TYPE OF REPORT } \\
\text { Topical Report }\end{array}$ \\
\hline & 7. PERIOD COVERED (Inclusive Dares) \\
\hline \multicolumn{2}{|c|}{$\begin{array}{l}\text { 8. PERFORMING ORGANIZATION - NAME AND ADDRESS (/f NRC, provide Division, Office or Region, U.S. Nuclear Regulatory Commission, and mailing address, if contractor, provide } \\
\text { name and mailling address) } \\
\text { Department of Hydrology and Water Resources }\end{array}$} \\
\hline \multicolumn{2}{|c|}{ 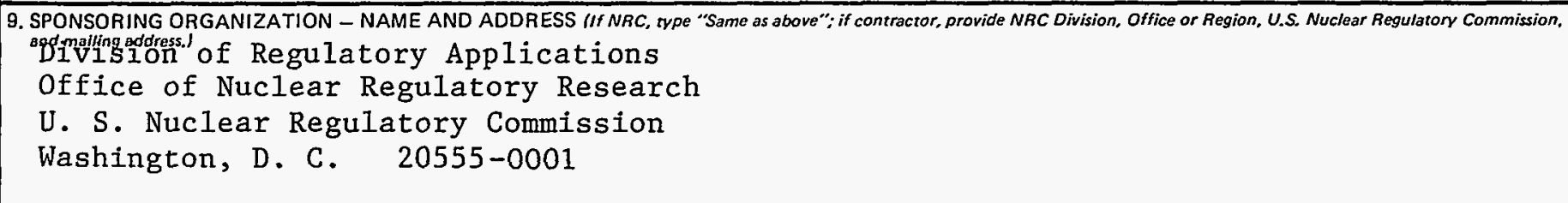 } \\
\hline \multicolumn{2}{|l|}{ 10. SUPPLEMENTARY NOTES } \\
\hline \multicolumn{2}{|c|}{$\begin{array}{l}\text { Wetting front instability is an important phenomenon affecting fluid flow and contaminant transport in } \\
\text { unsaturated soils and rocks. It causes the development of fingers which travel faster than would a uniform front } \\
\text { and thus bypass much of the medium. Water saturation and solute concentration in such fingers tend to be } \\
\text { higher than in the surrounding medium. During infiltration, fingering may cause unexpectedly rapid arrival of } \\
\text { water and solute at the water table. This notwithstanding, most models of subsurface flow and transport ignore } \\
\text { instability and fingering. In this report, we survey the literature to assess the extent to which this may or may } \\
\text { not be justified. Our overview covers experiments, theoretical studies, and computer simulations of instability } \\
\text { and fingering during immiscible two-phase flow and transport, with emphasis on infiltration into soils and } \\
\text { fractured rocks. Our description of instability in an ideal fracture (Hele-Shaw cell) includes an extension of } \\
\text { existing theory to fractures and interfaces having arbitrary orientations in space. Our discussion of instability in } \\
\text { porous media includes a slight but important correction of existing theory for the case of an inclined interface. } \\
\text { We conclude by outlining some potential directions for future research. Among these, we single out the effect } \\
\text { of soil and rock heterogeneities on instability and preferential flow as meriting special attention in the context } \\
\text { of nuclear waste storage in unsaturated media. }\end{array}$} \\
\hline \multirow{6}{*}{$\begin{array}{l}\text { Wetting front instability } \\
\text { Fingering } \\
\text { Infiltration } \\
\text { Immiscible displacement } \\
\text { Fracture flow }\end{array}$} & $\begin{array}{l}\text { 13. AVAILABILITY STATEMENT } \\
\text { Unlimited }\end{array}$ \\
\hline & 14. SECURITY CLASSIFIFATION \\
\hline & $\begin{array}{l}\text { (This Pagel } \\
\text { Unclassified }\end{array}$ \\
\hline & $\begin{array}{l}\text { (This Report) } \\
\text { Unclassified }\end{array}$ \\
\hline & 15. NUMBER OF PAGES \\
\hline & \\
\hline
\end{tabular}

